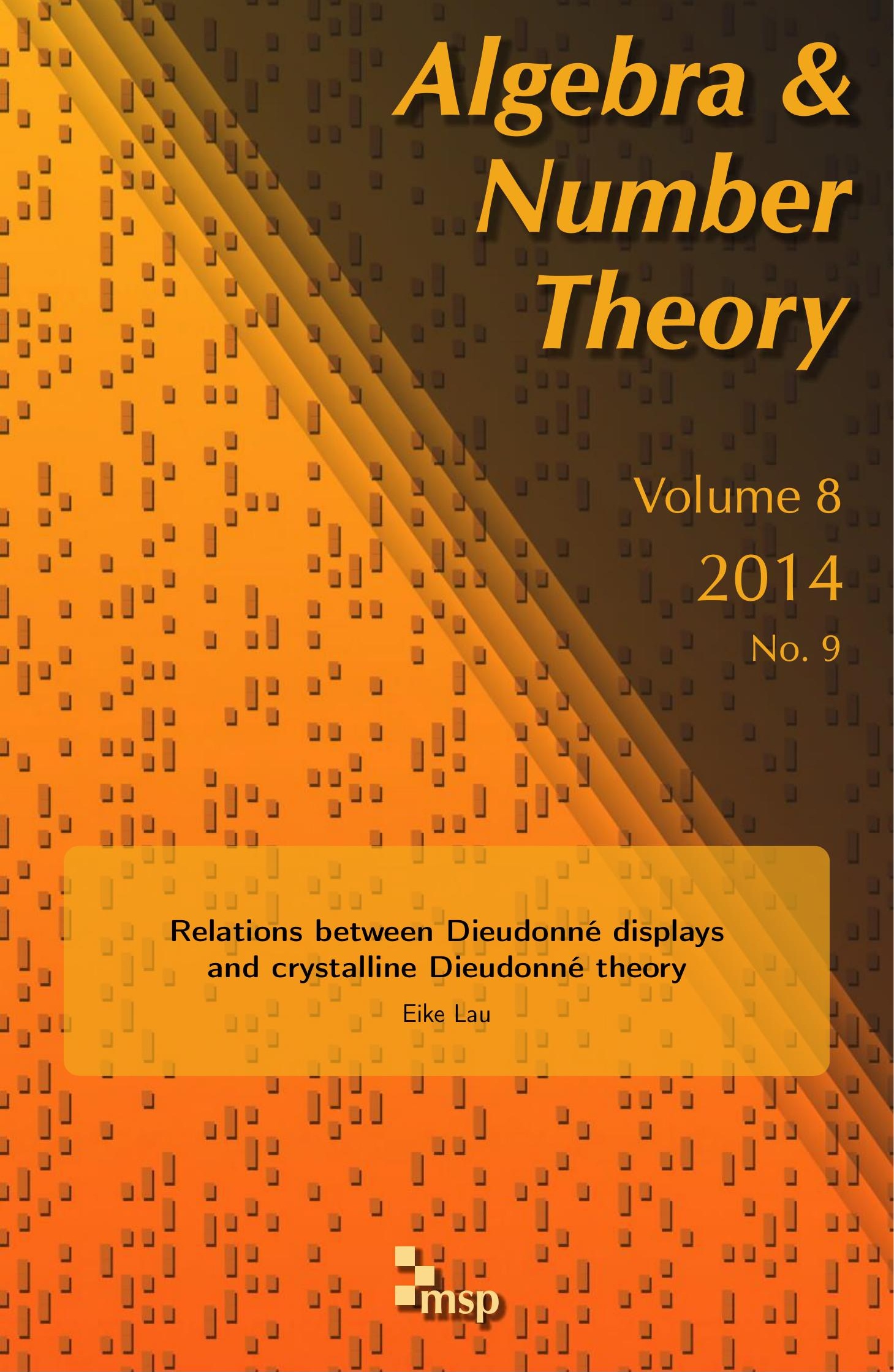




\title{
Relations between Dieudonné displays and crystalline Dieudonné theory
}

\author{
Eike Lau
}

We discuss the relation between crystalline Dieudonné theory and Dieudonné displays of $p$-divisible groups. The theory of Dieudonné displays is extended to the prime 2 without restriction, which implies that the classification of finite locally free group schemes by Breuil-Kisin modules holds for the prime 2 as well.

Introduction

1. The Zink ring 2204

2. Dieudonné displays 2213

3. From $p$-divisible groups to Dieudonné displays 2222

4. From 2-divisible groups to Dieudonné displays 2234

5. Equivalence of categories 2243

6. Breuil-Kisin modules 2244

7. Breuil-Kisin modules and crystals 2248

8. Rigidity of $p$-divisible groups 2254

9. The reverse functor 2256

Appendix: PD envelopes of regular immersions 2259

Acknowledgements 2261

References $\quad 2261$

\section{Introduction}

Formal $p$-divisible groups $G$ over a $p$-adically complete ring $R$ are classified by Zink's nilpotent displays [Zink 2002; Lau 2008]. These are projective modules over the ring of Witt vectors $W(R)$ equipped with a filtration and with certain Frobenius-linear operators. A central point of the theory is a description of the Dieudonné crystal of $G$ in terms of the nilpotent display associated to $G$.

Arbitrary $p$-divisible groups over $R$ can be classified by displays only when $R$ is a perfect ring. In certain cases, there is the following refinement.

Assume that $R$ is a local Artin ring with perfect residue field $k$ of characteristic $p$ and with maximal ideal $\mathcal{N}_{R}$. Then $W(R)$ has a unique subring $\mathbb{W}(R)$, here called

MSC2010: primary 14L05; secondary 14F30.

Keywords: $p$-divisible group, Dieudonné display, Dieudonné crystal. 
the Zink ring of $R$, which is stable under the Frobenius and which sits in an exact sequence

$$
0 \longrightarrow \widehat{W}\left(\mathcal{N}_{R}\right) \longrightarrow \mathbb{W}(R) \longrightarrow W(k) \longrightarrow 0,
$$

where $\widehat{W}$ means Witt vectors with only finitely many nonzero components. Let us call $R$ odd if $p>2$ or if $p$ annihilates $R$. The Verschiebung homomorphism $v$ of $W(R)$, which appears in the definition of displays, stabilises the subring $\mathbb{W}(R)$ if and only if $R$ is odd. In this case, Zink [2001a] defines Dieudonné displays over $R$ as displays with $\mathbb{W}(R)$ in place of $W(R)$, and shows that they classify all $p$-divisible groups over $R$.

The restriction for $p=2$ can be avoided with a small trick: The ring $\mathbb{W}(R)$ is always stable under the modified Verschiebung $\vee(x)=v\left(u_{0} x\right)$, where $u_{0} \in W(R)$ is the unit defined by the relation $v\left(u_{0}\right)=p-[p]$. This allows to define Dieudonné displays without assuming that $R$ is odd. It turns out that the Zink ring and Dieudonné displays can be defined for the following class of rings $R$, which we call admissible: the order of nilpotence of nilpotent elements of $R$ is bounded, and $R_{\text {red }}$ is a perfect ring of characteristic $p$.

Theorem A. For each admissible ring $R$ there is a functor

$\Phi_{R}:($-divisible groups over $R) \rightarrow($ Dieudonné displays over $R)$,

which is an equivalence of exact categories.

The equivalence easily extends to projective limits of admissible rings, which includes complete local rings with perfect residue field. If $R$ is perfect, the theorem says that $p$-divisible groups over $R$ are equivalent to Dieudonné modules. This is a result of Gabber, which is used in the proof. We repeat that for Artin rings (which is certainly the case of interest for most applications ${ }^{1}$ ), Theorem A is known when $R$ is odd; in this case, $\Phi_{R}$ is the inverse of the functor BT of [Zink 2001a] and [Lau 2009]. But the present construction of the functor $\Phi_{R}$ based on the crystalline Dieudonné module is new, and also gives the following second result.

Let $\mathbb{D}(G)$ denote the covariant Dieudonné crystal of a $p$-divisible group $G$. Following [Zink 2001b], to a Dieudonné display $\mathscr{P}$ over an admissible ring $R$ one can associate a crystal in locally free modules $\mathbb{D}(\mathscr{P})$.

Theorem B. For a p-divisible group $G$ over an admissible ring $R$ with associated Dieudonné display $\mathscr{P}=\Phi_{R}(G)$, there is a natural isomorphism

$$
\mathbb{D}(G) \cong \mathbb{D}(\mathscr{P}) .
$$

\footnotetext{
${ }^{1}$ In subsequent work, Dieudonné displays over a larger class of base rings will be used to study the image of the crystalline Dieudonné functor over 1.c.i. schemes.
} 
This compatibility was not known before and can be useful in applications; see for example [Viehmann and Wedhorn 2013]. Our proofs of Theorems A and B are closely related. The main point is to construct the functor $\Phi_{R}$ and variants of it. Let $\square_{R}$ be the kernel of the natural homomorphism $\mathbb{W}(R) \rightarrow R$.

First, if $R$ is an odd admissible ring, the ideal $\square_{R}$ carries natural divided powers. Thus the crystalline Dieudonné module of a $p$-divisible group over $R$ can be evaluated at $\mathbb{W}(R)$, which gives a filtered $F-V$-module over $\mathbb{W}(R)$. We show that this construction can be extended to a functor $\Phi_{R}$ as in Theorem A. This is not evident because a filtered $F$ - $V$-module does not in general determine a Dieudonné display. But the construction of $\Phi_{R}$ can be reduced to the case where $R$ is a universal deformation ring; then the Dieudonné display is determined uniquely because $p$ is not a zero divisor in $\mathbb{W}(R)$.

Next, for a divided power extension of admissible rings $S \rightarrow R$, one can define Dieudonné displays relative to $S \rightarrow R$, called triples in the work of Zink. They are modules over an extension $\mathbb{W}(S / R)$ of $\mathbb{W}(S)$. If $R$ is odd and the divided powers are compatible with the canonical divided powers of $p$, then the evaluation of the crystalline Dieudonné module at the divided power extension $\mathbb{W}(S / R) \rightarrow R$ can be extended to a functor

$$
\Phi_{S / R}:(p \text {-divisible groups over } R) \rightarrow(\text { Dieudonné displays for } S / R) .
$$

Again, this is not evident; the proof comes down to the fact that $p$ is not a zero divisor in the Zink ring of the divided power envelope of the diagonal of the universal deformation space of a $p$-divisible group. Once the functors $\Phi_{S / R}$ are known to exist, Theorems A and B for odd admissible rings are straightforward consequences.

Now let $R$ be an admissible ring which is not odd, so $p=2$. In this case, the preceding constructions do not apply directly because the ideal $\square_{R}$ does not in general carry divided powers. This changes when $\mathbb{W}(R)$ is replaced by the slightly larger $v$-stabilised Zink ring $\mathbb{W}^{+}(R)=\mathbb{W}(R)[v(1)]$. With an obvious definition of $v$-stabilised Dieudonné displays, we get a functor

$$
\left.\Phi_{R}^{+} \text {: (2-divisible groups over } R\right) \rightarrow(v \text {-stabilised Dieudonné displays over } R) \text {, }
$$

which is, however, not an equivalence. In order to construct a functor $\Phi_{R}$ as in Theorem $\mathrm{A}$, we have to descend from $\mathbb{W}^{+}(R)$ to $\mathbb{W}(R)$. This can be reduced to the minimal case where $2 \mathcal{N}_{R}=0$. Then the ideal $\square_{R}$ carries exceptional divided powers, which allows us to evaluate the crystalline Dieudonné module at $\mathbb{W}(R)$. In order to get the functor $\Phi_{R}$, we need some lift towards characteristic zero, which is provided by the fact that the exceptional divided powers exist on $\rrbracket_{R} /(v([4]))$ as soon as $4 \mathcal{N}_{R}=0$. Once $\Phi_{R}$ is known to exist in general, Theorems A and B follow again quite formally. 
Breuil-Kisin modules. Now let $R$ be a complete regular local ring with perfect residue field $k$ of characteristic $p$. Theorem A implies that the classification of $p$-divisible groups over $R$ by Breuil windows derived in [Vasiu and Zink 2010] and [Lau 2010] for odd $p$ holds for $p=2$ as well. Let us recall what this means: We write $R=\mathfrak{S} / E \mathfrak{S}$, where $\mathfrak{S}$ is a power series ring over $W(k)$ and where $E$ has constant term $p$; we also have to choose an appropriate Frobenius lift $\sigma$ on $\mathfrak{S}$. A Breuil window is a free $\mathfrak{S}$-module $Q$ equipped with an $\mathfrak{S}$-linear map $\phi: Q \rightarrow Q^{(\sigma)}$ whose cokernel is annihilated by $E$; this is equivalent to the notion of a Breuil-Kisin module. As usual, one also gets a classification of finite locally free $p$-group schemes over $R$.

In the case of discrete valuation rings this completes the proof of a conjecture of Breuil [1998], which was proved in [Kisin 2006] if $p$ is odd, and in [Kisin 2009] for connected $p$-divisible groups if $p=2$. Shortly after the first version of this article was posted, independent proofs of Breuil's conjecture by W. Kim [2012] and T. Liu [2013] appeared online.

Assume that $R$ has characteristic zero, and let $S$ be the $p$-adic completion of the divided power envelope of the ideal $E \mathfrak{S} \subset \mathfrak{S}$. As a consequence of Theorem B, we show that for a $p$-divisible group over $R$ the value of its crystalline Dieudonné module at $S$ coincides with the base change of its Breuil window under $\sigma: \mathfrak{S} \rightarrow S$. The functor BT. The original proof of Theorem A for odd local Artin rings in [Zink 2001a] depends on the construction of a functor BT from Dieudonné displays to $p$-divisible groups, which is a combination of the functor BT from nilpotent displays to formal $p$-divisible groups and a calculation of extensions. A modified construction of this functor is given in [Lau 2009]. Once the definition of Dieudonné displays for nonodd local Artin rings is available, all these arguments can be carried over almost literally to give an alternative proof of Theorem $\mathrm{A}$ in that case. In the present approach this construction serves only as an explicit description of the inverse of the functor $\Phi_{R}$; this is used in [Lau 2012].

All rings are commutative with a unit unless the contrary is stated. For a $p$ divisible group $G$, we denote by $\mathbb{D}(G)$ the covariant Dieudonné crystal.

\section{The Zink ring}

In this section we study the Zink ring $\mathbb{W}(R)$, which was introduced in [Zink 2001a] under the notation $\widehat{W}(R)$, and variants of $\mathbb{W}(R)$ in the presence of divided powers, following [Zink 2001b]. The definitions are stated in more generality, allowing arbitrary perfect rings instead of perfect fields. The modified Verschiebung $\vee$ for $p=2$ is new.

1A. Preliminaries. We fix a prime $p$. A commutative ring without unit $N$ is called bounded nilpotent if there is a number $n$ such that $x^{n}=0$ for every $x \in N$. We will consider the following type of base rings. 
Definition 1.1. A ring $R$ is called admissible if its nilradical $\mathcal{N}_{R}$ is bounded nilpotent and if $R_{\text {red }}=R / \mathcal{N}_{R}$ is a perfect ring of characteristic $p$.

Local Artin rings with perfect residue field are admissible. The ring $\mathcal{O}_{\mathbb{C}_{p}} / p$ is not admissible. We will also consider projective limits of admissible rings:

Definition 1.2. An admissible topological ring is a complete and separated topological ring $R$ with linear topology such that the ideal $\mathcal{N}_{R}$ of topologically nilpotent elements is open, the ring $R_{\text {red }}=R / \mathcal{N}_{R}$ is perfect of characteristic $p$, and for each open ideal $N$ of $R$ contained in $\mathcal{N}_{R}$, the quotient $\mathcal{N}_{R} / N$ is bounded nilpotent. Thus $R$ is the projective limit of the admissible rings $R / N$.

Examples of admissible topological rings include complete local rings with perfect residue field. Admissible topological rings in which $\mathcal{N}_{R}$ is not topologically nilpotent arise from divided power envelopes; see Lemma 1.13.

Notation 1.3. For a commutative, not necessarily unitary ring $A$, let $W(A)$ be the ring of $p$-typical Witt vectors of $A$. We write $f$ and $v$ for the Frobenius and Verschiebung of $W(A)$. Let $I_{A}=v(W(A))$, let $w_{i}: W(A) \rightarrow A$ be given by the $i$-th Witt polynomial, and let $\widehat{W}(A)$ be the group of all elements of $W(A)$ with nilpotent coefficients which are almost all zero.

Let us recall two well-known facts:

Lemma 1.4. Let $A$ be a perfect ring of characteristic $p$ and let $B$ be a ring with a bounded nilpotent ideal $J \subseteq B$. Every ring homomorphism $A \rightarrow B / J$ lifts to a unique ring homomorphism $W(A) \rightarrow B$.

Proof. See [Grothendieck 1974, Chapitre IV, Proposition 4.3]; the ideal $J$ there is assumed nilpotent, but the proof applies here as well.

Lemma 1.5 [Zink 2001b, Lemma 2.2]. Let $N$ be a nonunitary ring which is bounded nilpotent and annihilated by a power of $p$. Then $W(N)$ is bounded nilpotent and annihilated by a power of $p$.

1B. The Zink ring. Let $R$ be an admissible ring. By Lemma 1.4, the exact sequence

$$
0 \longrightarrow W\left(\mathcal{N}_{R}\right) \longrightarrow W(R) \longrightarrow W\left(R_{\mathrm{red}}\right) \longrightarrow 0
$$

has a unique ring homomorphism section $s: W\left(R_{\text {red }}\right) \rightarrow W(R)$, which is $f$ equivariant by its uniqueness. Let

$$
\mathbb{W}(R)=s W\left(R_{\text {red }}\right) \oplus \widehat{W}\left(\mathcal{N}_{R}\right) .
$$

Since $\hat{W}\left(\mathcal{N}_{R}\right)$ is an $f$-stable ideal of $W(R)$, the group $\mathbb{W}(R)$ is an $f$-stable subring of $W(R)$, which we call the Zink ring of $R$. 
Lemma 1.6. The ring $\mathbb{W}(R)$ is stable under the Verschiebung homomorphism $v: W(R) \rightarrow W(R)$ if and only if $p \geq 3$ or $p R=0$. In this case we have an exact sequence

$$
0 \longrightarrow \mathbb{W}(R) \stackrel{v}{\longrightarrow} \mathbb{W}(R) \stackrel{w_{0}}{\longrightarrow} R \longrightarrow 0 .
$$

Proof. See [Zink 2001a, Lemma 2]. For some $r \geq 0$, the $\operatorname{ring} R_{0}=\mathbb{Z} / p^{r} \mathbb{Z}$ is a subring of $R$, and we have $\mathbb{W}\left(R_{0}\right)=W\left(R_{0}\right) \cap \mathbb{W}(R)$. The calculation in [loc. cit.] shows that the element $v(1) \in W\left(R_{0}\right)$ lies in $\mathbb{W}\left(R_{0}\right)$ if and only if $p \geq 3$ or $r=1$. For $a \in W\left(R_{\text {red }}\right)$ we have $v(s(f(a)))=v(f(s(a)))=v(1) s(a)$. Since $\widehat{W}\left(\mathcal{N}_{R}\right)$ is stable under $v$ and since $f$ is surjective on $W\left(R_{\text {red }}\right)$, the first assertion of the lemma follows. The sequence is an extension of

$$
0 \longrightarrow W\left(R_{\text {red }}\right) \stackrel{v}{\longrightarrow} W\left(R_{\text {red }}\right) \longrightarrow R_{\text {red }} \longrightarrow 0
$$

and

$$
0 \longrightarrow \widehat{W}\left(\mathcal{N}_{R}\right) \stackrel{v}{\longrightarrow} \widehat{W}\left(\mathcal{N}_{R}\right) \longrightarrow \mathcal{N}_{R} \longrightarrow 0,
$$

which are both exact.

With a slight modification the exception at the prime 2 can be removed. The element $p-[p]$ of $W\left(\mathbb{Z}_{p}\right)$ lies in the image of $v$ because it maps to zero in $\mathbb{Z}_{p}$. Moreover, $v^{-1}(p-[p])$ maps to 1 in $W\left(\mathbb{F}_{p}\right)$, so this element is a unit in $W\left(\mathbb{Z}_{p}\right)$. We define

$$
u_{0}= \begin{cases}v^{-1}(2-[2]) & \text { if } p=2, \\ 1 & \text { if } p \geq 3 .\end{cases}
$$

The image of $u_{0}$ in $W(R)$ is also denoted by $u_{0}$. For $x \in W(R)$, let

$$
\vee(x)=v\left(u_{0} x\right) .
$$

One could also take $u_{0}=v^{-1}(p-[p])$ for all $p$, which would allow us to state some results more uniformly, but for odd $p$ this would be overcomplicated.

Lemma 1.7. The ring $\mathbb{W}(R)$ is stable under $\vee: W(R) \rightarrow W(R)$, and there is an exact sequence

$$
0 \longrightarrow \mathbb{W}(R) \stackrel{\vee}{\longrightarrow} \mathbb{W}(R) \stackrel{w_{0}}{\longrightarrow} R \longrightarrow 0 .
$$

Proof. By Lemma 1.6, we can assume that $p=2$. For $a \in W\left(R_{\text {red }}\right)$, we have $v(s(f(a)))=v\left(u_{0} f(s(a))\right)=v\left(u_{0}\right) s(a)=(2-[2]) s(a)$, which lies in $\mathbb{W}(R)$. Since $\widehat{W}\left(\mathcal{N}_{R}\right)$ is stable under $\vee$ and since $f$ is surjective on $W\left(R_{\text {red }}\right)$, it follows that $\mathbb{W}(R)$ is stable under $\vee$. The sequence is an extension of

$$
0 \longrightarrow W\left(R_{\text {red }}\right) \stackrel{\vee}{\longrightarrow} W\left(R_{\text {red }}\right) \longrightarrow R_{\text {red }} \longrightarrow 0
$$

and

$$
0 \longrightarrow \widehat{W}\left(\mathcal{N}_{R}\right) \stackrel{\vee}{\longrightarrow} \widehat{W}\left(\mathcal{N}_{R}\right) \longrightarrow \mathcal{N}_{R} \longrightarrow 0 .
$$

They are exact because in both cases $\vee=v \circ u_{0}$, where $u_{0}$ acts bijectively. 
1C. The enlarged Zink ring. Let us recall the logarithm of the Witt ring. For a divided power extension of rings $(B \rightarrow R, \delta$ ) with kernel $\mathfrak{b} \subseteq B$, the $\delta$-divided Witt polynomials define an isomorphism of $W(B)$-modules

$$
\log : W(\mathfrak{b}) \cong \mathfrak{b}^{\mathbb{N}}
$$

where $x \in W(B)$ acts on $\mathfrak{b}^{\mathbb{N}}$ by $\left[b_{0}, b_{1}, \ldots\right] \mapsto\left[w_{0}(x) b_{0}, w_{1}(x) b_{1}, \ldots\right]$. The Frobenius and Verschiebung of $W(\mathfrak{b})$ act on $\mathfrak{b}^{\mathbb{N}}$ by

$$
f\left(\left[b_{0}, b_{1}, \ldots\right]\right)=\left[p b_{1}, p b_{2}, \ldots\right], \quad v\left(\left[b_{0}, b_{1}, \ldots\right]\right)=\left[0, b_{0}, b_{1}, \ldots\right] .
$$

Moreover, Log induces an injective map $\widehat{W}(\mathfrak{b}) \rightarrow \mathfrak{b}^{(\mathbb{N})}$, which is bijective when the divided powers $\delta$ are nilpotent; see [Zink 2002, (149)] and the subsequent discussion. In general, let

$$
\widetilde{W}(\mathfrak{b})=\log ^{-1}\left(\mathfrak{b}^{(\mathbb{N})}\right) .
$$

This is an $f$-stable and $v$-stable ideal of $W(B)$ containing $\widehat{W}(\mathfrak{b})$.

Assume now that $(B \rightarrow R, \delta)$ is a divided power extension of admissible rings (it suffices to assume that $R$ is admissible and that $p$ is nilpotent in $B$, because then $\mathfrak{b}$ is bounded nilpotent due to the divided powers, so $B$ is admissible as well). Let

$$
\mathbb{W}(B, \delta)=\mathbb{W}(B)+\widetilde{W}(\mathfrak{b})
$$

This is an $f$-stable subring of $W(B)$, which we call the enlarged Zink ring of $B$ with respect to the divided power ideal $(\mathfrak{b}, \delta)$. We also write $\mathbb{W}(B / R)$ for $\mathbb{W}(B, \delta)$. If the divided powers $\delta$ are nilpotent then $\mathbb{W}(B, \delta)=\mathbb{W}(B)$. We have the following analogues of Lemmas 1.7 and 1.6:

Lemma 1.8. The ring $\mathbb{W}(B, \delta)$ is stable under $\vee: W(R) \rightarrow W(R)$, and there is an exact sequence

$$
0 \longrightarrow \mathbb{W}(B, \delta) \stackrel{\vee}{\longrightarrow} \mathbb{W}(B, \delta) \stackrel{w_{0}}{\longrightarrow} B \longrightarrow 0 .
$$

Proof. The ring $\mathbb{W}(B, \delta)$ is stable under $\vee$, because $\mathbb{W}(B)$ and $\tilde{W}(\mathfrak{b})$ are; see Lemma 1.7. We have $\mathbb{W}(B, \delta) / \widetilde{W}(\mathfrak{b})=\mathbb{W}(R)$. Thus, the exact sequence follows from the exactness of $0 \longrightarrow \tilde{W}(\mathfrak{b}) \stackrel{\vee}{\longrightarrow} \widetilde{\mathbb{W}}(\mathfrak{b}) \longrightarrow \mathfrak{b} \longrightarrow 0$ together with the exact sequence of Lemma 1.7.

Lemma 1.9. The ring $\mathbb{W}(B, \delta)$ is stable under $v: W(R) \rightarrow W(R)$ if $p \geq 3$, or if $p \in \mathfrak{b}$ and the divided powers $\delta$ on $\mathfrak{b}$ induce the canonical divided powers on $p B$. In this case we have an exact sequence

$$
0 \longrightarrow \mathbb{W}(B, \delta) \stackrel{v}{\longrightarrow} \mathbb{W}(B, \delta) \stackrel{w_{0}}{\longrightarrow} B \longrightarrow 0 .
$$


Proof. If $p \geq 3$ then $\mathbb{W}(B, \delta)$ is stable under $v$ because $\mathbb{W}(B)$ and $\tilde{W}(\mathfrak{b})$ are stable under $v$; see Lemma 1.6. Assume that $p \in \mathfrak{b}$ and that $\delta$ induces the canonical divided powers on $p B$. Let $\xi=p-v(1) \in W(B)$. This element lies in $W(p B) \subseteq W(\mathfrak{b})$ and satisfies $\log (\xi)=[p, 0,0, \ldots]$. Thus $\xi \in \tilde{W}(\mathfrak{b})$, which implies that $v(1) \in \mathbb{W}(B, \delta)$. Using this, the proof of Lemma 1.6 shows that $\mathbb{W}(B, \delta)$ is stable under $v$. The exact sequence follows as usual.

1D. The $v$-stabilised Zink ring. Assume that $p=2$. For an admissible ring $R$, let $\gamma$ be the canonical divided powers on the ideal $2 R$. We denote the associated enlarged Zink ring by

$$
\mathbb{W}^{+}(R)=\mathbb{W}(R, \gamma)=\mathbb{W}(R)+\widetilde{W}(2 R) \subseteq W(R) .
$$

The kernel of the projection $\mathbb{W}^{+}(R) \rightarrow W\left(R_{\text {red }}\right)$ will be denoted $\widehat{W}^{+}\left(\mathcal{N}_{R}\right)$. In view of the following lemma, we call $\mathbb{W}^{+}(R)$ the $v$-stabilised Zink ring.

Lemma 1.10. Let $p=2$. We have

$$
\mathbb{W}^{+}(R)=\mathbb{W}(R)+\mathbb{W}(R) v(1) .
$$

The ring $\mathbb{W}^{+}(R)$ is equal to $\mathbb{W}(R)$ if and only if $2 R=0$. The $\mathbb{W}(R)$-module $\mathbb{W}^{+}(R) / \mathbb{W}(R)$ is an $R_{\mathrm{red}}$-module generated by $v(1)$.

Proof. By Lemma 1.9, we have $v(1) \in \mathbb{W}^{+}(R)$. Clearly $2 R=0$ implies that $\mathbb{W}^{+}(R)=\mathbb{W}(R)$. In general, we consider the filtration

$$
W\left(2 \mathcal{N}_{R}\right) \subseteq W(2 R) \subseteq W(R)
$$

and the graded modules for the induced filtrations on $\mathbb{W}(R)$ and $\mathbb{W}^{+}(R)$. First, the restriction of the divided powers $\gamma$ to the ideal $2 \mathcal{N}_{R}$ is nilpotent, which implies that

$$
\mathbb{W}^{+}(R) \cap W\left(2 \mathcal{N}_{R}\right)=\tilde{W}\left(2 \mathcal{N}_{R}\right)=\widehat{W}\left(2 \mathcal{N}_{R}\right)=\mathbb{W}(R) \cap W\left(2 \mathcal{N}_{R}\right) .
$$

Next we have $\mathbb{W}^{+}(R / 2 R)=\mathbb{W}(R / 2 R)$, or equivalently

$$
\mathbb{W}^{+}(R) / \mathbb{W}^{+}(R) \cap W(2 R)=\mathbb{W}(R) / \mathbb{W}(R) \cap W(2 R) .
$$

Let $\mathfrak{c}=2 R / 2 \mathcal{N}_{R}$. By the preceding remarks, we have an isomorphism

$$
\mathbb{W}^{+}(R) / \mathbb{W}(R) \cong \tilde{W}(\mathfrak{c}) / \widehat{W}(\mathfrak{c}) .
$$

This is an $R / \mathcal{N}_{R}$-module. Assume that $2 R \neq 0$, which implies that $\mathfrak{c} \neq 0$. For some ideal $\mathcal{N}_{R} \subseteq \mathfrak{b} \subseteq R$, multiplication by 2 induces an isomorphism $R / \mathfrak{b} \cong \mathfrak{c}$. Modulo 2 , the divided Witt polynomials are $\tilde{w}_{i}(x) \equiv \gamma_{2}\left(x_{i-1}\right)+x_{i}$, so the isomorphism $\log : W(\mathfrak{c}) \rightarrow \mathfrak{c}^{\mathbb{N}}$ takes the form

$$
\log \left(2 a_{0}, 2 a_{1}, \ldots\right)=2\left[a_{0}, a_{0}^{2}+a_{1}, a_{1}^{2}+a_{2}, a_{2}^{2}+a_{3}, \ldots\right],
$$


with $a_{i} \in R / \mathfrak{b}$. It follows that $\widetilde{W}(\mathfrak{c}) / \widehat{W}(\mathfrak{c})$ can be identified with the direct limit of the Frobenius homomorphism $R / \mathfrak{b} \rightarrow R / \mathfrak{b} \rightarrow \cdots$, which is isomorphic to $R / \sqrt{\mathfrak{b}}$. Under this identification, the element $\xi=2-v(1)$ of $\widetilde{W}(\mathfrak{c})$ maps to 1 in $R / \sqrt{\mathfrak{b}}$, because we have $\log (\xi)=[2,0, \ldots]$. Hence $\mathbb{W}^{+}(R) / \mathbb{W}(R)$ is generated by $v(1)$, with annihilator $\sqrt{\mathfrak{b}}$.

Assume again that $p=2$. Let $(B \rightarrow R, \delta)$ be a divided power extension of admissible rings with kernel $\mathfrak{b} \subseteq B$ such that $\delta$ is compatible with the canonical divided powers $\gamma$ on $2 B$. Let $\delta^{+}$be the divided powers on $\mathfrak{b}^{+}=\mathfrak{b}+2 B$ that extend $\delta$ and $\gamma$. In this case, we write

$$
\mathbb{W}^{+}(B, \delta)=\mathbb{W}\left(B, \delta^{+}\right)=\mathbb{W}(B)+\tilde{W}\left(\mathfrak{b}^{+}\right) .
$$

Clearly $\mathbb{W}(B, \delta) \subseteq \mathbb{W}^{+}(B, \delta) \supseteq \mathbb{W}^{+}(B)$. If the divided powers on $\mathfrak{b}^{+} / 2 B$ induced by $\delta$ are nilpotent, then $\mathbb{W}^{+}(B, \delta)=\mathbb{W}^{+}(B)$.

1E. Passing to the limit. The preceding considerations carry over to the topological case as follows. For an admissible topological ring $R$, let

$$
\mathbb{W}(R)=\lim _{N} \mathbb{W}(R / N)
$$

the limit taken over all open ideals $N$ of $R$ with $N \subseteq \mathcal{N}_{R}$. Then Lemmas 1.6 and 1.7 hold for admissible topological rings. The enlarged Zink ring can be defined for topological divided power extensions in the following sense.

Definition 1.11. Let $B$ and $R$ be admissible topological rings. A topological divided power extension is a surjective ring homomorphism $B \rightarrow R$ whose kernel $\mathfrak{b}$ is equipped with divided powers $\delta$ such that $\mathfrak{b}$ is closed in $B$, the topology of $R$ is the quotient topology of $B / \mathfrak{b}$, and the linear topology of $B$ is induced by open ideals $N$ for which $N \cap \mathfrak{b}$ is stable under $\delta$. Let $\delta / N$ be the divided powers on $N / N \cap \mathfrak{b}$ induced by $\delta$. We say that $\delta$ is topologically compatible with the canonical divided powers of $p$ if the topology of $B$ is induced by open ideals $N$ such that $\delta / N$ is defined and compatible with the canonical divided powers of $p$.

Remark 1.12. The existence of divided powers on $\mathfrak{b}$ implies that $\mathfrak{b} \subseteq \mathcal{N}_{B}$. If $B$ is a noetherian complete local ring, then every ideal $\mathfrak{b}$ of $B$ is closed; moreover, if $\mathfrak{b}$ is given, for each $n$ there is an open ideal $N \subseteq \mathfrak{m}_{B}^{n}$ such that $\mathfrak{b} \cap N$ is stable under arbitrary divided powers $\delta$ on $\mathfrak{b}$. Indeed, by Artin-Rees there is an $l$ with $\mathfrak{m}_{B}^{n} \mathfrak{b} \supseteq \mathfrak{m}_{B}^{l} \cap \mathfrak{b}$; then take $N=\mathfrak{m}_{B}^{n} \mathfrak{b}+\mathfrak{m}_{B}^{l}$, which implies that $\mathfrak{b} \cap N=\mathfrak{m}_{B}^{n} \mathfrak{b}$.

Given a topological divided power extension of admissible topological rings $(B \rightarrow R, \delta)$ with kernel $\mathfrak{b} \subseteq B$, we define

$$
\mathbb{W}(B, \delta)=\lim _{N} \mathbb{W}(B / N, \delta / N)
$$


where $N$ runs through the open ideals of $B$ contained in $\mathcal{N}_{B}$ such that $N \cap \mathfrak{b}$ is stable under $\delta$. Lemmas 1.8 and 1.9 hold in the topological case.

Assume that $p=2$. Then for an admissible topological ring, we put

$$
\mathbb{W}^{+}(R)=\underset{N}{\lim } \mathbb{W}^{+}(R / N),
$$

the limit taken over all open ideals $N$ of $R$ contained in $\mathcal{N}_{R}$. If $(B \rightarrow R, \delta)$ is a topological divided power extension of admissible topological rings which is topologically compatible with the canonical divided powers of 2 , we can define

$$
\mathbb{W}^{+}(B, \delta)=\underset{N}{\lim } \mathbb{W}^{+}(B / N, \delta / N)
$$

where $N$ runs through the open ideals of $B$ contained in $\mathcal{N}_{B}$ such that $\delta / N$ is defined and compatible with the canonical divided powers of 2 .

The following example of admissible topological rings is used in Section 3:

Lemma 1.13. Let $R$ be a ring which is $I$-adically complete for an ideal $I \subseteq R$ such that $K=R / I$ is a perfect ring of characteristic $p$. Assume that $I=J+p R$ for an ideal $J \subseteq R$ such that $R / J^{n}$ has no p-torsion for each $n$. For a projective $R$-module $t$ of finite type, we consider the complete symmetric algebra

$$
R \llbracket t \rrbracket=\prod_{n \geq 0} \operatorname{Sym}_{R}^{n}(t) .
$$

Let $(\mathfrak{a} \subseteq S, \delta)$ be the divided power envelope of the ideal $t R \llbracket t \rrbracket \subseteq R \llbracket t \rrbracket$, and let $\hat{S}$ be the I-adic completion of $S$. Then:

(i) $\hat{S} \rightarrow R$ is naturally a topological divided power extension of admissible topological rings which is topologically compatible with the canonical divided powers of $p$.

(ii) $\widehat{S}$ has no p-torsion.

Proof. Let $\bar{R}_{n}=R /\left(p^{n} R+J^{n}\right)$ and $\bar{S}_{n}=S \otimes_{R} R_{n}$. We have $S=R \oplus \mathfrak{a}$ and thus $\bar{S}_{n}=\bar{R}_{n} \oplus \overline{\mathfrak{a}}_{n}$ with $\overline{\mathfrak{a}}_{n}=\mathfrak{a} \otimes_{R} \bar{R}_{n}$; moreover, the ideal $\overline{\mathfrak{a}}_{n}$ carries divided powers $\delta_{n}$ induced by $\delta$; see [Berthelot 1974, Chapitre I, Proposition 1.7.1]. In particular, $\bar{S}_{n}$ is admissible. Since $\widehat{S} \rightarrow R$ is the projective limit over $n$ of $\bar{S}_{n} \rightarrow \bar{R}_{n}$, to prove (i) it suffices to show that $\delta_{n}$ is compatible with the canonical divided powers of $p$. Now, Spec $R \rightarrow \operatorname{Spec} R \llbracket t \rrbracket$ is a regular immersion by Lemma A.3, and thus $S$ is flat over $R$ by Proposition A.1. Since $R$ has no $p$-torsion the same holds for $S$, so the divided powers on $\mathfrak{a}$ extend canonically to the ideal $\mathfrak{b}=\mathfrak{a}+p S$. We have $S / \mathfrak{b}=R / p R$. The assumptions imply that $\operatorname{Tor}_{1}^{R}\left(R / J^{n}, R / p R\right)$ is zero. Hence there is an exact sequence

$$
0 \longrightarrow \mathfrak{b} / J^{n} \mathfrak{b} \longrightarrow S / J^{n} S \longrightarrow R /\left(p R+J^{n}\right) \longrightarrow 0,
$$


which in turn gives an exact sequence

$$
0 \longrightarrow\left(\mathfrak{b} / J^{n} \mathfrak{b}\right) / p^{n}\left(S / J^{n} S\right) \longrightarrow S /\left(J^{n} S+p^{n} S\right) \longrightarrow R /\left(p R+J^{n}\right) \longrightarrow 0 .
$$

In both sequences the kernels carry divided powers which extend the canonical divided powers of $p$, since the ideals $J^{n} \mathfrak{b}$ of $S$ and $p^{n}\left(S / J^{n} S\right)$ of $S / J^{n} S$ are stable under the given divided powers. Thus the divided powers $\delta_{n}$ on $\overline{\mathfrak{a}}_{n}$ are compatible with the canonical divided powers of $p$, which proves (i).

Let $S_{n}=S / J^{n} S$, and let $\hat{S}_{n}$ be its $p$-adic completion. Since $S$ is flat over $R$ and since $R / J^{n}$ has no $p$-torsion, $S_{n}$ and $\widehat{S}_{n}$ have no $p$-torsion. Using that $\widehat{S}=\lim _{n} \widehat{S}_{n}$, it follows that $\widehat{S}$ has no $p$-torsion, which proves (ii).

1F. Completeness. For an admissible ring $R$, the Zink ring $\mathbb{W}(R)$ is $p$-adically complete. Indeed, $W\left(R_{\text {red }}\right)$ is $p$-adically complete, and $\widehat{W}\left(\mathcal{N}_{R}\right)$ is annihilated by a power of $p$ because this holds for $W\left(\mathcal{N}_{R}\right)$ by Lemma 1.5. The following topological variant of this fact seems to be less obvious:

Proposition 1.14. Let $R$ be an I-adically complete ring such that the ideal $I$ is finitely generated and $K=R / I$ is a perfect ring of characteristic $p$. Then the ring $\mathbb{W}(R)$ is $p$-adically complete. If $p=2$, the ring $\mathbb{W}^{+}(R)$ is $p$-adically complete too.

This is similar to [Zink 2002, Proposition 3], which says that $W(R)$ is $p$-adically complete if this holds for $R$.

Proof. The ring $W(R)$ is $p$-adically separated, because this holds for each $W\left(R / I^{n}\right)$. Thus $\mathbb{W}(R)$ is $p$-adically separated too. Let $S=W(K) \llbracket t_{1}, \ldots, t_{r} \rrbracket$, and let $S \rightarrow R$ be a homomorphism which maps $t_{1}, \ldots, t_{r}$ to a set of generators of $I / I^{2}$. Then $S \rightarrow R$ is surjective, and so is $\mathbb{W}(S) \rightarrow \mathbb{W}(R)$. Since $\mathbb{W}(R)$ is $p$-adically separated, in order to show that $\mathbb{W}(R)$ is $p$-adically complete we may assume that $R=S$. Consider the ideals $J_{n}=p^{n} W(R)+W\left(I^{n}\right)$ of $W(R)$ and $\rrbracket_{n}=\mathbb{W}(R) \cap J_{n}$ of $\mathbb{W}(R)$. Then

$$
W(R) / J_{n}=W_{n}(K) \oplus W\left(I / I^{n}\right), \quad \mathbb{W}(R) / \mathbb{J}_{n}=W_{n}(K) \oplus \widehat{W}\left(I / I^{n}\right) .
$$

It follows that $W(R)$ and $\mathbb{W}(R)$ are complete and separated for the linear topologies generated by the ideals $J_{n}$ and $\rrbracket_{n}$, respectively; moreover, $\mathbb{W}(R)$ is closed in $W(R)$. The ring $W(R)$ is also complete and separated for the linear topology generated by the ideals $J_{n, m}^{\prime}=\operatorname{Ker}\left(W(R) \rightarrow W_{m}\left(R / I^{n}\right)\right)$. The $J$-topology is finer than the $J^{\prime}$-topology because $J_{2 n} \subseteq J_{n, n}^{\prime}$.

We claim that for each $r \geq 1$ the ideal $p^{r} W(R)$ of $W(R)$ is closed in the $J^{\prime}$ topology. This is a variant of [Zink 2002, Lemma 6] with essentially the same proof. First, for $s \geq 1$, an element $x=\left(x_{0}, \ldots, x_{m}\right)$ of $W_{m+1}(R)$ satisfies $x_{i} \in I^{s}$ for all $i$ if and only if $w_{i}(x) \in I^{i+s}$ for all $i$; see the proof of [Zink 2002, Lemma 4]. Then the proof of Lemma 5 in that work shows that an element $x \in W_{m}(R)$ is divisible 
by $p^{r}$ if and only if for each $s$ the image $\bar{x} \in W_{m}\left(R / I^{s}\right)$ is divisible by $p^{r}$. Using this, the claim follows from the proof of [Zink 2002, Lemma 6].

Thus $p^{r} W(R)$ is closed in the finer $J$-topology as well. Assume that we have

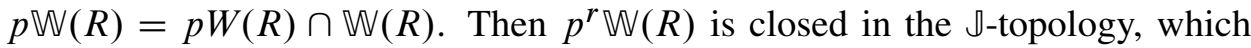
implies that $\mathbb{W}(R)$ is $p$-adically complete; see [Zink 2002, Lemma 7]. Thus for $p \geq 3$, the proof is completed by Lemma 1.15 below. For $p=2$ the same reasoning shows that $\mathbb{W}^{+}(R)$ is $p$-adically complete. Now $\mathbb{W}^{+}(R) / \mathbb{W}(R)$ is isomorphic to $K$ as abelian groups by the proof of Lemma 1.10. We get exact sequences

$$
0 \longrightarrow K \longrightarrow \mathbb{W}(R) / 2^{n} \mathbb{W}(R) \longrightarrow \mathbb{W}^{+}(R) / 2^{n} \mathbb{W}^{+}(R) \longrightarrow K \longrightarrow 0,
$$

where the transition maps from $n+1$ to $n$ are zero on the left-hand $K$ and the identity on the right-hand $K$. It follows that $\mathbb{W}(R)$ is $p$-adically complete as well.

Lemma 1.15. For a perfect ring $K$ of characteristic $p$, let $R=W(K) \llbracket t_{1}, \ldots, t_{r} \rrbracket$, with the $\left(p, t_{1}, \ldots, t_{r}\right)$-adic topology. If $p \geq 3$ then

$$
p W(R) \cap \mathbb{W}(R)=p \mathbb{W}(R) .
$$

If $p=2$ then

$$
2 W(R) \cap \mathbb{W}^{+}(R)=2 \mathbb{W}^{+}(R) .
$$

Proof. Assume $p=2$. Let $I$ be the kernel of $R \rightarrow K$ and let $\bar{I}=I / p R$. The filtration $0 \subseteq W(p R) \subseteq W(I) \subseteq W(R)$ induces a filtration of $\mathbb{W}(R)$ with successive quotients $\tilde{W}(p R):=\lim _{n} \tilde{W}\left(p R / I^{n} p R\right)$ and $\hat{W}(\bar{I}):=\lim _{n} \hat{W}\left(\bar{I} / \bar{I}^{n}\right)$ and $W(K)$. To prove the lemma it suffices to show that

$$
p W(\bar{I}) \cap \widehat{W}(\bar{I})=p \widehat{W}(\bar{I})
$$

and

$$
p W(p R) \cap \tilde{W}(p R)=p \tilde{W}(p R) .
$$

The first equality holds since multiplication by $p$ on $W(\bar{I})$ is given by $\left(a_{0}, a_{1}, \ldots\right) \mapsto$ $\left(0, a_{0}^{p}, a_{1}^{p}, \ldots\right)$, and for $a \in \bar{I}$ with $a^{p} \in \bar{I}^{p n}$ we have $a \in \bar{I}^{n}$. The second equality holds because the isomorphism $\log : W(p R) \cong(p R)^{\mathbb{N}}$ induces an isomorphism between $\widetilde{W}(p R)$ and the group of all sequences in $(p R)^{\mathbb{N}}$ that converge to zero $I$-adically. The proof for $p \geq 3$ is similar.

1G. Divided powers. In Section 3, we will use that the augmentation ideals of the Zink ring and its variants carry natural divided powers, with some exception when $p=2$; see also Section 4A.

Let us first recall the canonical divided powers on the Witt ring. If $R$ is a $\mathbb{Z}_{(p)^{-}}$ algebra, then $W(R)$ is a $\mathbb{Z}_{(p)}$-algebra as well, and the ideal $I_{R}$ carries divided powers $\gamma$ which are determined by $(p-1) ! \gamma_{p}(v(x))=p^{p-2} v\left(x^{p}\right)$. Assume that $(B \rightarrow R, \delta)$ is a divided power extension of $\mathbb{Z}_{(p)}$-algebras with kernel $\mathfrak{b} \subseteq B$. Let $I_{B / R}$ be the kernel of $W(B) \rightarrow R$. If $i: \mathfrak{b} \rightarrow W(\mathfrak{b})$ is defined by $\log (i(b))=$ 
$[b, 0,0, \ldots]$, we have $I_{B / R}=I_{B} \oplus i(\mathfrak{b})$, and the divided powers $\gamma$ on $I_{B}$ extend to divided powers $\gamma^{\prime}=\gamma \oplus \delta$ on $I_{B / R}$ such that $\gamma_{n}^{\prime}(i(b))=i\left(\delta_{n}(b)\right)$ for $b \in \mathfrak{b}$. If $p \in \mathfrak{b}$ and if $\delta$ extends the canonical divided powers of $p$, then $\gamma \oplus \delta$ extends the canonical divided powers of $p$, and $f$ preserves $\gamma \oplus \delta$. This is clear when $B$ has no $p$-torsion; the general case follows because $(B \rightarrow R)$ can be written as the quotient of a divided power extension $\left(B^{\prime} \rightarrow R^{\prime}\right)$, where $B^{\prime}$ is the divided power algebra of a free module over a polynomial ring $R^{\prime \prime}$ over $\mathbb{Z}_{(p)}$, and $R^{\prime}=R^{\prime \prime} / p R^{\prime \prime}$.

These facts extend to the Zink ring as follows:

Lemma 1.16. Let $\square \subseteq \mathbb{W}$ be one of the following:

(i) $\llbracket=\rrbracket_{R}$ and $\mathbb{W}=\mathbb{W}(R)$ for an admissible ring $R$ with $p \geq 3$.

(ii) $\mathbb{\square}=\square_{R}^{+}$and $\mathbb{W}=\mathbb{W}^{+}(R)$ for an admissible ring $R$ with $p=2$.

Then the divided powers $\gamma$ on $I_{R}$ induce divided powers on $\square$.

Proof. Since $\mathbb{W}$ is a $\mathbb{Z}_{(p)}$-algebra, it suffices to show that $\square$ is stable under the map $\gamma_{p}: I_{R} \rightarrow I_{R}$, which is true because $\mathbb{\square}=v(\mathbb{W})$ by Lemmas 1.6 and 1.9.

Lemma 1.17. Let $(B \rightarrow R, \delta)$ be a divided power extension of admissible rings with kernel $\mathfrak{b} \subseteq B$, and let $\rrbracket_{B / R}$ be the kernel of $\mathbb{W}(B, \delta) \rightarrow R$. Assume that $p \geq 3$; or that $p=2$ and $p \in \mathfrak{b}$ and $\delta$ extends the canonical divided powers of $p$. Then the divided powers $\gamma \oplus \delta$ on $I_{B / R}$ induce divided powers on $\square_{B / R}$. If $p \in \mathfrak{b}$ and if $\delta$ extends the canonical divided powers of $p$, then the divided powers on $\rrbracket_{B / R}$ induced by $\gamma \oplus \delta$ extend the canonical divided powers of $p$ and are preserved by $f$.

Proof. Let $\mathbb{\square}_{B}^{\prime}$ be the kernel of $\mathbb{W}(B / R) \rightarrow B$. Then $\rrbracket_{B / R}=\mathbb{\square}_{B}^{\prime} \oplus i(\mathfrak{b})$, and we have $\mathbb{\square}_{B}^{\prime}=v(\mathbb{W}(B / R))$ by Lemma 1.9. Thus $\mathbb{\square}_{B}^{\prime}$ is stable under $\gamma$, and $\square_{B / R}$ is stable under $\gamma \oplus \delta$. The second assertion follows from the corresponding fact for the Witt ring.

\section{Dieudonné displays}

In this section, Dieudonné displays and a number of variants related to divided power extensions are defined. We use the formalism of frames and windows introduced in [Lau 2010]. First of all, let us recall a well-known fact:

Lemma 2.1. Let $A$ be a commutative, not necessarily unitary ring. For $x \in W(A)$ we have $f(x) \equiv x^{p}$ modulo $p W(A)$. Similarly, for $x \in \widehat{W}(A)$ we have $f(x) \equiv x^{p}$ modulo $p \hat{W}(A)$.

Proof. For $x \in W(R)$ write $x=\left[x_{0}\right]+v(y)$ with $x_{0} \in R$ and $y \in W(R)$. Then $f(x) \equiv\left[x_{0}^{p}\right] \equiv x^{p}$ modulo $p W(R)$ because $f v=p$ and $v(y)^{p}=p^{p-1} v\left(y^{p}\right)$. The same calculation applies with $\widehat{W}$ in place of $W$. 
2A. Frames and windows. We recall the notion of frames and windows from [Lau 2010], with some additions. A preframe is a quintuple

$$
\mathcal{F}=\left(S, I, R, \sigma, \sigma_{1}\right)
$$

where $S$ and $R=S / I$ are rings, where $\sigma: S \rightarrow S$ is a ring endomorphism with $\sigma(a) \equiv a^{p}$ modulo $p S$, and where $\sigma_{1}: I \rightarrow S$ is a $\sigma$-linear map of $S$-modules whose image generates $S$ as an $S$-module. Then there is a unique element $\theta \in S$ with $\sigma(a)=\theta \sigma_{1}(a)$ for $a \in I$. The preframe $\mathcal{F}$ is called a frame if

$$
I+p S \subseteq \operatorname{Rad}(S) .
$$

If, in addition, all projective $R$-modules of finite type can be lifted to projective $S$ modules, then $\mathcal{F}$ is called a lifting frame.

A homomorphism of preframes or frames $\alpha: \mathcal{F} \rightarrow \mathcal{F}^{\prime}$ is a ring homomorphism $\alpha: S \rightarrow S^{\prime}$ with $\alpha(I) \subseteq I^{\prime}$ such that $\sigma^{\prime} \alpha=\alpha \sigma$ and $\sigma_{1}^{\prime} \alpha=u \cdot \alpha \sigma_{1}$ for a unit $u \in S^{\prime}$, which is then determined by $\alpha$. It also follows that $\alpha(\theta)=u \theta^{\prime}$. We say that $\alpha$ is a $u$-homomorphism of preframes or frames. If $u=1$ then $\alpha$ is called strict.

Now let $\mathcal{F}$ be a frame. An $\mathcal{F}$-window is a quadruple

$$
\mathscr{P}=\left(P, Q, F, F_{1}\right)
$$

where $P$ is a projective $S$-module of finite type with a submodule $Q$ such that there exists a decomposition of $S$-modules $P=L \oplus T$ with $Q=L \oplus I T$, called a normal decomposition, and where $F: P \rightarrow P$ and $F_{1}: Q \rightarrow P$ are $\sigma$-linear maps of $S$-modules with

$$
F_{1}(a x)=\sigma_{1}(a) F(x)
$$

for $a \in I$ and $x \in P$; we also assume that $F_{1}(Q)$ generates $P$ as an $S$-module. Then $F(x)=\theta F_{1}(x)$ for $x \in Q$. If $F$ is a lifting frame, every pair $(P, Q)$ such that $P$ is a projective $S$-module of finite type and $P / Q$ is a projective $R$-module admits a normal decomposition. In general, for given $(P, Q)$ together with a normal decomposition $P=L \oplus T$, giving $\sigma$-linear maps $\left(F, F_{1}\right)$ which make an $\mathcal{F}$-window $\mathscr{P}$ is equivalent to giving a $\sigma$-linear isomorphism

$$
\Psi: L \oplus T \rightarrow P
$$

defined by $F_{1}$ on $L$ and by $F$ on $T$. The triple $(L, T, \Psi)$ is called a normal representation of $\mathscr{P}$.

A frame homomorphism $\alpha: \mathcal{F} \rightarrow \mathcal{F}^{\prime}$ induces a base change functor $\alpha_{*}$ from $\mathcal{F}$-windows to $\mathcal{F}^{\prime}$-windows. In terms of normal representations, it is given by

$$
(L, T, \Psi) \mapsto\left(S^{\prime} \otimes_{S} L, S^{\prime} \otimes_{S} T, \Psi^{\prime}\right)
$$

with $\Psi^{\prime}\left(s^{\prime} \otimes l\right)=u \sigma^{\prime}\left(s^{\prime}\right) \otimes \Psi(l)$ and $\Psi^{\prime}\left(s^{\prime} \otimes t\right)=\sigma^{\prime}\left(s^{\prime}\right) \otimes \Psi(t)$. 
A frame homomorphism $\alpha: \mathcal{F} \rightarrow \mathcal{F}^{\prime}$ is called crystalline if the functor $\alpha_{*}$ is an equivalence of categories. For reference, we recall [Lau 2010, Theorem 3.2]:

Theorem 2.2. Let $\alpha: \mathcal{F} \rightarrow \mathcal{F}^{\prime}$ be a homomorphism of frames which induces an isomorphism $R \cong R^{\prime}$ and a surjection $S \rightarrow S^{\prime}$ with kernel $\mathfrak{a}$. We assume that there is a finite filtration of ideals $\mathfrak{a}=\mathfrak{a}_{0} \supseteq \cdots \supseteq \mathfrak{a}_{n}=0$ with $\sigma_{1}\left(\mathfrak{a}_{i}\right) \subseteq \mathfrak{a}_{i}$ and $\sigma\left(\mathfrak{a}_{i}\right) \subseteq \mathfrak{a}_{i+1}$, that $\sigma_{1}$ is elementwise nilpotent on each $\mathfrak{a}_{i} / \mathfrak{a}_{i+1}$, and that all projective $S^{\prime}$-modules of finite type lift to projective $S$-modules of finite type. Then $\alpha$ is crystalline.

Let us recall the operator $V^{\sharp}$ of a window. For an $S$-module $M$ we write $M^{(\sigma)}=S \otimes_{\sigma, S} M$. A filtered $F-V$-module over $\mathcal{F}$ is a quadruple

$$
\left(P, Q, F^{\sharp}, V^{\sharp}\right)
$$

where $P$ is a projective $S$-module of finite type, $Q$ is a submodule of $P$ such that $P / Q$ is projective over $R$, and $F^{\sharp}: P^{(\sigma)} \rightarrow P$ and $V^{\sharp}: P \rightarrow P^{(\sigma)}$ are $S$-linear maps with $F^{\sharp} V^{\sharp}=\theta$ and $V^{\sharp} F^{\sharp}=\theta$.

Lemma 2.3. There is a natural functor from $\mathcal{F}$-windows to filtered $F$ - $V$-modules over $\mathcal{F}$, which is fully faithful if $\theta$ is not a zero divisor in $S$.

Proof. The functor is $\left(P, Q, F, F_{1}\right) \mapsto\left(P, Q, F^{\sharp}, V^{\sharp}\right)$, where $F^{\sharp}$ is the linearisation of $F$, and $V^{\sharp}$ is the unique $S$-linear map such that $V^{\sharp}\left(F_{1}(x)\right)=1 \otimes x$ for $x \in Q$. Clearly this determines $V^{\sharp}$ if it exists. In terms of a normal representation $(L, T, \Psi)$ of $\mathscr{P}$, thus $P=L \oplus T$, one can define $V^{\sharp}=(1 \oplus \theta)\left(\Psi^{\sharp}\right)^{(-1)}$. The required relation $F^{\sharp} V^{\sharp}=\theta$ on $P$ is equivalent to $F^{\sharp} V^{\sharp} F_{1}=\theta F_{1}$ on $Q$, which is clear since $\theta F_{1}=F$. The required relation $V^{\sharp} F^{\sharp}=\theta$ on $P^{(\sigma)}$ holds if and only if it holds after multiplication with $\sigma_{1}(a)$ for all $a \in I$. For $x \in P$ we calculate $\sigma_{1}(a) V^{\sharp} F^{\sharp}(1 \otimes x)=V^{\sharp} F_{1}(a x)=\sigma(a) \otimes x=\theta \sigma_{1}(a)(1 \otimes x)$.

Assume that $\theta$ is not a zero divisor in $S$. It suffices to show that the forgetful functors from windows to triples $(P, Q, F)$ and from filtered $F-V$-modules to triples $\left(P, Q, F^{\sharp}\right)$ are fully faithful. In the first case this holds because $\theta F_{1}=F$. In the second case, for an endomorphism $\alpha$ of $P$ with $\alpha F^{\sharp}=F^{\sharp} \alpha^{(\sigma)}$ we calculate $V^{\sharp} \alpha \theta=$ $V^{\sharp} \alpha F^{\sharp} V^{\sharp}=V^{\sharp} F^{\sharp} \alpha^{(\sigma)} V^{\sharp}=\theta \alpha^{(\sigma)} V^{\sharp}$, which implies that $V^{\sharp} \alpha=\alpha^{(\sigma)} V^{\sharp}$.

Finally, we recall the duality formalism. Let $\mathcal{F}$ denote the $\mathcal{F}$-window $\left(S, I, \sigma, \sigma_{1}\right)$. A bilinear form between $\mathcal{F}$-windows

$$
\beta: \mathscr{P} \times \mathscr{P}^{\prime} \rightarrow \mathcal{F}
$$

is an $S$-bilinear map $\beta: P \times P^{\prime} \rightarrow S$ such that $\beta\left(Q \times Q^{\prime}\right) \subseteq I$ and $\beta\left(F_{1} x, F_{1}^{\prime} x^{\prime}\right)=$ $\sigma_{1}\left(\beta\left(x, x^{\prime}\right)\right)$ for $x \in Q$ and $x^{\prime} \in Q^{\prime}$. For each $\mathscr{P}$, the functor $\mathscr{P}^{\prime} \mapsto \operatorname{Bil}\left(\mathscr{P} \times \mathscr{P}^{\prime}, \mathcal{F}\right)$ is represented by an $\mathcal{F}$-window $\mathscr{P}^{t}$, called the dual of $\mathscr{P}$. The tautological bilinear form $\mathscr{P} \times \mathscr{P}^{t} \rightarrow S$ is a perfect bilinear map $P \times P^{t} \rightarrow S$. There is a bijection between normal representations $P=L \oplus T$ and $P^{t}=L^{t} \oplus T^{t}$ determined by 
$\left\langle L, L^{t}\right\rangle=0=\left\langle T, T^{t}\right\rangle$. The associated operators $\Psi: P \rightarrow P$ and $\Psi^{t}: P^{t} \rightarrow P^{t}$ are related by $\left\langle\Psi x, \Psi^{t} x^{\prime}\right\rangle=\sigma\left\langle x, x^{\prime}\right\rangle$.

There is also an obvious duality of filtered $F-V$-modules over $\mathcal{F}$ : the dual of $\mathcal{M}=\left(P, Q, F^{\sharp}, V^{\sharp}\right)$ is $\mathcal{M}^{t}=\left(P^{*}, Q^{\prime}, V^{\sharp *}, F^{\sharp *}\right)$, where $P^{*}=\operatorname{Hom}_{S}(P, S)$ and $Q^{\prime}$ is the submodule of all $y$ in $P^{*}$ with $y(Q) \subseteq I$. It is easy to see that the functor in Lemma 2.3 preserves duality.

2B. Frames associated to the Witt ring. For an arbitrary ring $R$ let $f_{1}: I_{R} \rightarrow$ $W(R)$ be the inverse of the Verschiebung $v$. Then

$$
\mathscr{W}_{R}=\left(W(R), I_{R}, R, f, f_{1}\right)
$$

is a preframe with $\theta=p$. If $R$ is $p$-adically complete, $\mathscr{W}_{R}$ is a lifting frame because $W(R)$ is $I_{R}$-adically complete by [Zink 2002, Proposition 3], and windows over $\mathscr{W}_{R}$ are displays over $R$.

For a divided power extension of rings $(B \rightarrow R, \delta)$ with kernel $\mathfrak{b} \in B$, one can define a preframe

$$
\mathscr{W}_{B / R}=\left(W(B), I_{B / R}, R, f, \tilde{f}_{1}\right)
$$

with $I_{B / R}=I_{B}+W(\mathfrak{b})$ such that $\tilde{f}_{1}: I_{B / R} \rightarrow W(B)$ is the unique extension of $f_{1}$ whose restriction to $W(\mathfrak{b})$ is given by $\left[a_{0}, a_{1}, a_{2}, \ldots\right] \mapsto\left[a_{1}, a_{2}, \ldots\right]$ in logarithmic coordinates; see Section $1 \mathrm{C}$. The projection $\mathscr{W}_{B} \rightarrow \mathscr{W}_{R}$ factors into strict preframe homomorphisms $\mathscr{W}_{B} \rightarrow \mathscr{W}_{B / R} \rightarrow \mathscr{W}_{R}$.

As a special case, assume that $R$ is a perfect ring of characteristic $p$. Then $f$ is an automorphism of $W(R)$, and $I_{R}=p W(R)$. Let us define a Dieudonné module over $R$ to be a triple $(P, F, V)$ where $P$ is a projective $W(R)$-module of finite type equipped with an $f$-linear endomorphism $F$ and an $f^{-1}$-linear endomorphism $V$ such that $F V=p$, or, equivalently, $V F=p$.

Lemma 2.4. Displays over a perfect ring $R$ are equivalent to Dieudonné modules over $R$.

Proof. To a display $\left(P, Q, F, F_{1}\right)$ we associate the Dieudonné module $(P, F, V)$, where the linearisation of $V: P \rightarrow P$ is the operator $V^{\sharp}$ defined in Lemma 2.3. Then $V F_{1}: Q \rightarrow P$ is the inclusion. Here $F_{1}$ is surjective since $f$ is bijective. Thus $Q=V(P)$, and the functor is fully faithful; see Lemma 2.3. It remains to show that for every Dieudonné module $(P, F, V)$ the $R$-module $M=P / V(P)$ is projective. For $\mathfrak{p} \in \operatorname{Spec} R$ let $\ell_{M}(\mathfrak{p})$ be the dimension of the fibre of $M$ at $\mathfrak{p}$. Let $N=P / F(P)$. Then $\ell_{M}+\ell_{N}=\ell_{P / p P}$ as functions on Spec $R$. Since $M$ and $N$ are of finite type and since $P / p P$ is projective, the functions $\ell_{M}$ and $\ell_{N}$ are upper semicontinuous, and $\ell_{P / p P}$ is locally constant. It follows that $\ell_{M}$ is locally constant, which implies that $M$ is projective because $R$ is reduced. 
2C. Dieudonné frames. For an admissible ring $R$ in the sense of Definition 1.1, let $\rrbracket_{R}$ be the kernel of $w_{0}: \mathbb{W}(R) \rightarrow R$, and let $\mathbb{\Xi}_{1}: \square_{R} \rightarrow \mathbb{W}(R)$ be the inverse of $\vee$, which is well-defined by Lemma 1.7. If $p$ is odd, then $\vee=v$ and $\mathbb{t}_{1}=f_{1}$.

Lemma 2.5. The quintuple

$$
\mathscr{D}_{R}=\left(\mathbb{W}_{R}, \rrbracket_{R}, R, f, \mathbb{E}_{1}\right)
$$

is a lifting frame.

We call $\mathscr{D}_{R}$ the Dieudonné frame associated to $R$.

Proof. In order that $\mathscr{D}_{R}$ is a preframe we need that $f(a) \equiv a^{p}$ modulo $p \mathbb{W}(R)$ for $a \in \mathbb{W}(R)$, which follows from Lemma 2.1 applied to $W\left(R_{\text {red }}\right)$ and to $\widehat{W}\left(\mathcal{N}_{R}\right)$. Since $\widehat{W}\left(\mathcal{N}_{R}\right)$ is a nilideal by Lemma 1.5 and since the quotient $\mathbb{W}(R) / \widehat{W}\left(\mathcal{N}_{R}\right)=$ $W\left(R_{\text {red }}\right)$ is $p$-adically complete with $p W\left(R_{\text {red }}\right)=I_{R_{\text {red }}}$, the kernel of $\mathbb{W}(R) \rightarrow R_{\text {red }}$

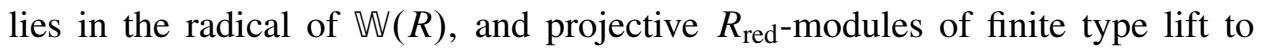
projective $\mathbb{W}(R)$-modules of finite type. It follows that $\mathscr{D}_{R}$ is a lifting frame.

The inclusion $\mathbb{W}(R) \rightarrow W(R)$ is a $u_{0}$-homomorphism of frames $\mathscr{D}_{R} \rightarrow \mathscr{W}_{R}$. Thus for $\mathscr{D}_{R}$ we have $\theta=p$ if $p$ is odd and $\theta=2 u_{0}=2-[4]$ if $p=2$.

Definition 2.6. A Dieudonné display over $R$ is a window over $\mathscr{D}_{R}$.

Thus a Dieudonné display is a quadruple $\mathscr{P}=\left(P, Q, F, F_{1}\right)$ where $P$ is a projective $\mathbb{W}(R)$-module of finite type with a filtration $\rrbracket_{R} P \subseteq Q \subseteq P$ such that $P / Q$ is a projective $R$-module, $F: P \rightarrow P$ and $F_{1}: Q \rightarrow P$ are $f$-linear maps with $F_{1}(a x)=\mathbb{G}_{1}(a) F(x)$ for $a \in \mathbb{\square}_{R}$ and $x \in P$, and $F_{1}(Q)$ generates $P$. We write

$$
\operatorname{Lie}(\mathscr{P})=P / Q \text {. }
$$

The height of $\mathscr{P}$ is the rank of the $\mathbb{W}(R)$-module $P$, and the dimension of $\mathscr{P}$ is the rank of the $R$-module $\operatorname{Lie}(\mathscr{P})$, both viewed as locally constant functions on Spec $R$. As in the case of general frames, we also denote by $\mathscr{D}_{R}$ the Dieudonné display $\left(\mathbb{W}(R), \mathbb{\square}_{R}, f, \mathbb{f}_{1}\right)$ over $R$.

2D. Relative Dieudonné frames. Let $(B \rightarrow R, \delta)$ be a divided power extension of admissible rings with kernel $\mathfrak{b} \subseteq B$. Let $\mathbb{W}(B / R)=\mathbb{W}(B, \delta)$ as in Section 1C and let $\rrbracket_{B / R}$ be the kernel of the projection $\mathbb{W}(B / R) \rightarrow R$; thus

$$
\rrbracket_{B / R}=\rrbracket_{B}+\tilde{W}(\mathfrak{b}) .
$$

Lemma 2.7. There is a unique extension of $\mathbb{E}_{1}: \rrbracket_{B} \rightarrow \mathbb{W}(B)$ to an $f$-linear map

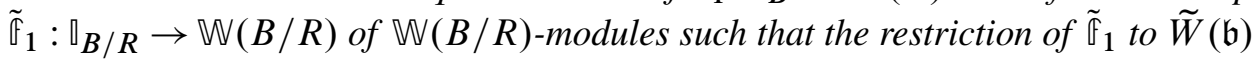
is given by

$$
\tilde{\mathbb{\mathbb { V }}}_{1}\left(\left[a_{0}, a_{1}, a_{2}, \ldots\right]\right)=\left[w_{0}\left(u_{0}^{-1}\right) a_{1}, w_{1}\left(u_{0}^{-1}\right) a_{2}, \ldots\right]
$$


in logarithmic coordinates. The quintuple

$$
\mathscr{D}_{B / R}=\mathscr{D}_{B / R, \delta}=\left(\mathbb{W}(B / R), \rrbracket_{B / R}, R, f, \tilde{\mathbb{E}}_{1}\right)
$$

is a lifting frame.

Proof. Clearly $\tilde{\mathbb{T}}_{1}$ is determined by (2-1). Let $\mathbb{\mathbb { B }}_{B}^{\prime}$ be the kernel of $\mathbb{W}(B / R) \rightarrow B$. By Lemma 1.8, the inverse of $\vee$ is an $f$-linear map $\mathbb{\mathbb { V }}_{1}^{\prime}: \mathbb{\square}_{B}^{\prime} \rightarrow \mathbb{W}(B / R)$ which extends $\mathbb{Q}_{1}$. In logarithmic coordinates, the restriction of $\vee$ to $W(\mathfrak{b})$ is given by $\left[a_{0}, a_{1}, \ldots\right] \mapsto\left[0, w_{0}\left(u_{0}\right) a_{0}, w_{1}\left(u_{0}\right) a_{1}, \ldots\right]$. Thus $\mathbb{\mathbb { E }}_{1}^{\prime}$ extends to the desired $\tilde{\mathbb{F}}_{1}$. As in the proof of Lemma 2.5, the kernel of $\mathbb{W}(B / R) \rightarrow R_{\text {red }}$ lies in the radical of $\mathbb{W}(B / R)$, and projective $R_{\text {red }}$-modules of finite type lift to $\mathbb{W}(B / R)$.

We call $\mathscr{D}_{B / R}$ the relative Dieudonné frame associated to the divided power extension $(B / R, \delta)$, and $\mathscr{D}_{B / R}$-windows are called Dieudonné displays for $B / R$. There are natural strict frame homomorphisms

$$
\mathscr{D}_{B} \longrightarrow \mathscr{D}_{B / R} \longrightarrow \mathscr{D}_{R}
$$

If the divided powers $\delta$ are nilpotent, then $\mathbb{W}(B)=\mathbb{W}(B / R)$.

Proposition 2.8. The frame homomorphism $\mathscr{D}_{B / R} \rightarrow \mathscr{D}_{R}$ is crystalline.

Proof. This follows from Theorem 2.2. Indeed, let $\mathfrak{a}$ denote the kernel of the surjective homomorphism $\mathbb{W}(B / R) \rightarrow \mathbb{W}(R)$; thus $\mathfrak{a}=\widetilde{W}(\mathfrak{b}) \cong \mathfrak{b}^{(\mathbb{N})}$. The endomorphism $\tilde{\mathbb{F}}_{1}$ of $\mathfrak{a}$ is elementwise nilpotent by (2-1). The required filtration of $\mathfrak{a}$ can be taken to be $\mathfrak{a}_{i}=p^{i} \mathfrak{a}$; this is a finite filtration by Lemma 1.5. We have $\tilde{\mathbb{G}}_{1}\left(\mathfrak{a}_{i}\right)=\mathfrak{a}_{i}$ by $(2-1)$, and $f\left(\mathfrak{a}_{i}\right)=\mathfrak{a}_{i+1}$ because the endomorphism $f$ of $\mathfrak{a}$ is given by $\left[a_{0}, a_{1}, \ldots\right] \mapsto\left[p a_{1}, p a_{2}, \ldots\right]$ in logarithmic coordinates.

2E. v-stabilised Dieudonné frames. Assume that $p=2$. The preceding constructions can be repeated with $\mathbb{W}^{+}$and $v$ in place of $\mathbb{W}$ and $v$. More precisely, for an admissible ring $R$, let $\square_{R}^{+}$be the kernel of $\mathbb{W}^{+}(R) \rightarrow R$ and let $f_{1}: \square_{R}^{+} \rightarrow \mathbb{W}^{+}(R)$ be the inverse of $v$, which is well-defined by Lemma 1.9. The $v$-stabilised Dieudonné frame associated to $R$ is defined as

$$
\mathscr{D}_{R}^{+}=\left(\mathbb{W}^{+}(R), \square_{R}^{+}, R, f, f_{1}\right) .
$$

This is a lifting frame by the proof of Lemma 2.5. The inclusion $\mathbb{W}(R) \rightarrow \mathbb{W}^{+}(R)$ is a $u_{0}$-homomorphism of frames $\mathscr{D}_{R} \rightarrow \mathscr{D}_{R}^{+}$, which is invertible if and only if $2 R=0$. Windows over $\mathscr{D}_{R}^{+}$are called $v$-stabilised Dieudonné displays over $R$.

Assume again that $p=2$, and let $(B \rightarrow R, \delta)$ be a divided power extension of admissible rings with kernel $\mathfrak{b} \subseteq B$ which is compatible with the canonical divided powers of 2. Let $\square_{B / R}^{+}$be the kernel of the natural map $\mathbb{W}_{B / R}^{+} \rightarrow R$; thus

$$
\rrbracket_{B / R}^{+}=\rrbracket_{B}^{+}+\tilde{W}(\mathfrak{b}) .
$$


There is a unique extension of $f_{1}: \square_{B}^{+} \rightarrow \mathbb{W}^{+}(B)$ to an $f$-linear map of $\mathbb{W}^{+}(B / R)$ modules $\tilde{f}_{1}: \square_{B / R}^{+} \rightarrow \mathbb{W}^{+}(B / R)$ such that its restriction to $\tilde{W}(\mathfrak{b})$ is given by $\left[a_{0}, a_{1}, a_{2}, \ldots\right] \mapsto\left[a_{1}, a_{2}, \ldots\right]$ in logarithmic coordinates, and the quintuple

$$
\mathscr{D}_{B / R}^{+}=\left(\mathbb{W}^{+}(B / R), \square_{B / R}^{+}, R, f, \tilde{f}_{1}\right)
$$

is a lifting frame. This follows from the proof of Lemma 2.7. We have a $u_{0^{-}}$ homomorphism of frames $\mathscr{D}_{B / R} \rightarrow \mathscr{D}_{B / R}^{+}$, which is invertible if and only if $2 R=0$, and strict frame homomorphisms

$$
\mathscr{D}_{B}^{+} \longrightarrow \mathscr{D}_{B / R}^{+} \longrightarrow \mathscr{D}_{R}^{+} .
$$

If the divided powers induced by $\delta$ on $(\mathfrak{b}+2 B) / 2 B$ are nilpotent, then $\mathbb{W}^{+}(B)$ is equal to $\mathbb{W}^{+}(B / R)$.

Corollary 2.9. The frame homomorphism $\mathscr{D}_{B / R}^{+} \rightarrow \mathscr{D}_{R}^{+}$is crystalline.

Proof. This follows from the proof of Proposition 2.8.

2F. The crystals associated to Dieudonné displays. Let $R$ be an admissible ring. We denote the category of divided power extensions ( $\operatorname{Spec} A \rightarrow \operatorname{Spec} B, \delta$ ), where $A$ is an $R$-algebra which is an admissible ring, and where $p$ is nilpotent in $B$, by Cris adm $_{\text {ad }}(R)$. Then the kernel of $B \rightarrow A$ is bounded nilpotent, so $B$ is an admissible ring as well.

Let $\mathscr{P}$ be a Dieudonné display over $R$. For $\left(\operatorname{Spec} A \rightarrow \operatorname{Spec} B, \delta\right.$ ) in $\operatorname{Cris}_{\text {adm }}(R)$, we denote the base change of $\mathscr{P}$ to $A$ by $\mathscr{P}_{A}$ and the unique Dieudonné display for $B / A$ which lifts $\mathscr{P}_{A}$ by

$$
\mathscr{P}_{B / A}=\left(P_{B / A}, Q_{B / A}, F, F_{1}\right) ;
$$

see Proposition 2.8. A homomorphism of divided power extensions of admissible rings $\alpha:(B \rightarrow A, \delta) \rightarrow\left(B^{\prime} \rightarrow A^{\prime}, \delta^{\prime}\right)$ induces a frame homomorphism $\mathscr{D}_{\alpha}: \mathscr{D}_{B / A} \rightarrow$ $\mathscr{D}_{B^{\prime} / A^{\prime}}$, and we have a natural isomorphism

$$
\left(\mathscr{D}_{\alpha}\right)_{*}\left(\mathscr{P}_{B / A}\right) \cong \mathscr{P}_{B^{\prime} / A^{\prime}}
$$

In more sophisticated terms, this can be expressed as follows: The frames $\mathscr{D}_{B / A}$ form a presheaf of frames $\mathscr{D}_{* *}$ on $\operatorname{Cris}_{\text {adm }}(R)$, and Proposition 2.8 implies that the category of Dieudonné displays over $R$ is equivalent to the category of crystals in

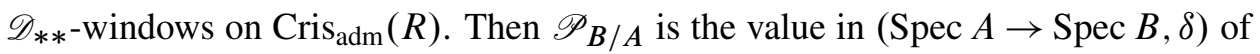
the crystal associated to $\mathscr{P}$.

For a Dieudonné display $\mathscr{P}=\left(P, Q, F, F_{1}\right)$ over $R$, we define the Witt crystal $\mathbb{K}(\mathscr{P})$ on $\mathrm{Cris}_{\mathrm{adm}}(R)$ by

$$
\mathbb{R}(\mathscr{P})_{B / A}=P_{B / A}
$$


This is a projective $\mathbb{W}(B / A)$-module of finite type. The Dieudonné crystal $\mathbb{D}(\mathscr{P})$ on $\operatorname{Cris}_{\mathrm{adm}}(R)$ is defined by

$$
\mathbb{D}(\mathscr{P})_{B / A}=P_{B / A} \otimes_{\mathbb{W}(B / A)} B .
$$

This is a projective $B$-module of finite type. The Hodge filtration of $\mathscr{P}$ is the submodule

$$
Q / \rrbracket_{R} P \subseteq P / \rrbracket_{R} P=\mathbb{D}(\mathscr{P})_{R / R}
$$

Corollary 2.10. Let $(B \rightarrow R, \delta)$ be a nilpotent divided power extension of admissible rings. The category of Dieudonné displays over $B$ is equivalent to the category of Dieudonné displays $\mathscr{P}$ over $R$ together with a lift of the Hodge filtration of $\mathscr{P}$ to a direct summand of $\mathbb{D}(\mathscr{P})_{B / R}$.

Proof. If the divided powers are nilpotent, then $\mathbb{W}(B / R)=\mathbb{W}(B)$, and lifts of windows under the frame homomorphism $\mathscr{D}_{B} \rightarrow \mathscr{D}_{B / R}$ are in bijection with lifts of the Hodge filtration.

The preceding definitions have a $v$-stabilised variant. Let $\operatorname{Cris}_{\text {adm }}\left(R / \mathbb{Z}_{p}\right)$ be the full subcategory of $\operatorname{Cris}_{\text {adm }}(R)$ where the divided powers are compatible with the canonical divided powers of $p$. Assume now that $p=2$, and let $\mathscr{P}^{+}$be a $v$-stabilised Dieudonné display over $R$, i.e., a window over $\mathscr{D}_{R}^{+}$. For $(\operatorname{Spec} A \rightarrow \operatorname{Spec} B, \delta$ ) in Cris adm $_{\text {ad }}\left(R / \mathbb{Z}_{2}\right)$ we denote by $\mathscr{P}_{A}^{+}$the base change of $\mathscr{P}^{+}$to $\mathscr{D}_{A}^{+}$and by

$$
\mathscr{P}_{B / A}^{+}=\left(P_{B / A}^{+}, Q_{B / A}^{+}, F, F_{1}\right)
$$

the unique lift of $\mathscr{P}_{A}^{+}$to a $\mathscr{D}_{B / A^{-}}^{+}$window, which exists by Corollary 2.9. The $v$ stabilised Witt crystal $\mathbb{K}^{+}\left(\mathscr{P}^{+}\right)$and the $v$-stabilised Dieudonné crystal $\mathbb{D}^{+}\left(\mathscr{P}^{+}\right)$ on $\operatorname{Cris}_{\mathrm{adm}}\left(R / \mathbb{Z}_{2}\right)$ are defined by $\mathbb{K}^{+}\left(\mathscr{P}^{+}\right)_{B / A}=P_{B / A}^{+}$and

$$
\mathbb{D}^{+}\left(\mathscr{P}^{+}\right)_{B / A}=P_{B / A}^{+} \otimes_{\mathbb{W}+(B / A)} B .
$$

Corollary 2.11. Assume that $p=2$. Let $(B \rightarrow R, \delta)$ be a divided power extension of admissible rings which is compatible with the canonical divided powers of 2 such that the divided powers induced by $\delta$ on the kernel of $B / 2 B \rightarrow R / 2 R$ are nilpotent. Then the category of $v$-stabilised Dieudonne displays over $B$ is equivalent to the category of $v$-stabilised Dieudonné displays $\mathscr{P}^{+}$over $R$ together with a lift of the Hodge filtration of $\mathscr{P}^{+}$to a direct summand of $\mathbb{D}^{+}\left(\mathscr{P}^{+}\right)_{B / R}$.

Proof. This is analogous to Corollary 2.10 , using that $\mathbb{W}^{+}(B / R)=\mathbb{W}^{+}(B)$ under the given assumptions on $\delta$; see the end of Section 1D.

Lemma 2.12. Let $\mathscr{P}$ be a Dieudonné display over an admissible ring $R$ with $p=2$, and let $\mathscr{P}^{+}$be its base change to $\mathscr{D}_{R}^{+}$. Then $\mathbb{D}\left(\mathscr{P}^{+}\right)$is naturally isomorphic to the restriction of $\mathbb{D}(\mathscr{P})$ to $\mathrm{Cris}_{\mathrm{adm}}\left(R / \mathbb{Z}_{2}\right)$. 


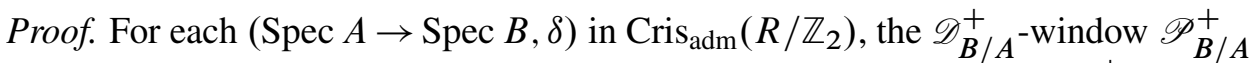
is the base change of $\mathscr{P}_{B / A}$ by the frame homomorphism $\mathscr{D}_{B / A} \rightarrow \mathscr{D}_{B / A}^{+}$by its uniqueness. The lemma follows easily.

Remark 2.13. Lemma 2.12 does not imply that the infinitesimal deformations of $\mathscr{P}$ and of $\mathscr{P}^{+}$coincide: Let $B$ be an admissible ring with $4 B=0$ and $2 B \neq 0$ and let $R=B / 2 B$. The ideal $2 B$ carries the canonical divided powers $\gamma$ and the trivial divided powers $\delta$. Corollary 2.10 applies to $(B \rightarrow R, \delta)$ but not to $(B \rightarrow R, \gamma)$, while Corollary 2.11 and Lemma 2.12 apply to $(B \rightarrow R, \gamma)$ but not to $(B \rightarrow R, \delta)$.

2G. Passing to the limit. The preceding considerations extend easily to the case of admissible topological rings with a countable base of topology. Let us begin with a standard lemma. For a ring $A$, let $\mathrm{V}(A)$ be the category of projective $A$-modules of finite type.

Lemma 2.14. Let $A=\lim _{n \in \mathbb{N}} A_{n}$ be an inverse limit of rings such that the transition maps $\pi_{n}: A_{n} \rightarrow{\overleftarrow{A_{n}}}_{n \in \mathbb{N}}$ are surjective with $\operatorname{Ker}\left(\pi_{n}\right) \subseteq \operatorname{Rad}\left(A_{n}\right)$. Then the natural functor $\rho: \mathrm{V}(A) \rightarrow \lim _{n} \mathrm{~V}\left(A_{n}\right)$ is an equivalence.

Proof. Since for $P \in \mathrm{V}(A)$ we have $P=\lim _{n}\left(P \otimes_{A} A_{n}\right)$, the functor $\rho$ is fully faithful. For a system of $P_{n} \in \mathrm{V}\left(A_{n}\right)$ with isomorphisms $P_{n} \otimes_{A} A_{n-1} \cong P_{n-1}$, we have to show that the $A$-module $P=\lim _{n} P_{n}$ lies in $\mathrm{V}(A)$. Choose a surjective homomorphism $q_{1}: A_{1}^{r} \rightarrow P_{1}$ and lift this to a compatible system of homomorphisms $q_{n}$ : $A_{n}^{r} \rightarrow P_{n}$. All the $q_{n}$ are surjective by Nakayama's Lemma. Let $S_{n}$ be the set of linear sections of $q_{n}$. Since $S_{n}$ carries a simply transitive action of $\operatorname{Hom}\left(P_{n}, \operatorname{Ker}\left(q_{n}\right)\right)$, the reduction maps $S_{n} \rightarrow S_{n-1}$ are surjective. Thus the limit map $q: A^{r} \rightarrow P$ has a section, and we have $P \in \mathrm{V}(A)$. This proves that $\rho$ is an equivalence.

For a ring $A$, let $\mathrm{BT}(A)$ be the category of $p$-divisible groups over $A$.

Lemma 2.15. For an inverse limit $A=\lim _{n} A_{n}$ as in Lemma 2.14, the natural functor $v: \mathrm{BT}(A) \rightarrow \lim _{n} \mathrm{BT}\left(A_{n}\right)$ is an equivalence.

Proof. See [Messing 1972, Chapter II, Lemma 4.16]. The functor $\rho$ of Lemma 2.14 preserves tensor products, and a complex $P \rightarrow P^{\prime} \rightarrow P^{\prime \prime} \rightarrow 0$ in $\mathrm{V}(A)$ is exact if and only if its reduction to $A_{1}$ is exact. As in [Messing 1972, Chapter II, Lemma 4.16] it follows that $v$ is an equivalence.

For an admissible topological ring $R$, let $\mathscr{D}_{R}=\lim _{N} \mathscr{D}_{R / N}$, where $N$ runs through the open ideals of $R$ contained in $\mathcal{N}_{R}$. As before, $\mathscr{D}_{R}$-windows are called Dieudonné displays over $R$.

Lemma 2.16. If $R$ is an admissible topological ring with a countable base of topology, then Dieudonné displays (or p-divisible groups) over $R$ are equivalent to compatible systems of Dieudonné displays (or p-divisible groups) over $R / N$ for each open ideal $N$ contained in $\mathcal{N}_{R}$. 
Proof. One can write $R=\lim _{n \in \mathbb{N}} R_{n}$ for a surjective system of admissible rings $R_{n}$ with $R_{\text {red }}=\left(R_{n}\right)_{\text {red }}$ for each $n$. Then the case of $p$-divisible groups follows from Lemma 2.15, and the case of Dieudonné displays follows from Lemma 2.14 applied to $R$ and to $\mathbb{W}(R)=\lim _{n} \mathbb{W}\left(R_{n}\right)$; here the successive kernels are nilideals due to

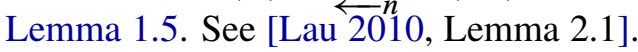

\section{From $p$-divisible groups to Dieudonné displays}

In this section we define a functor from $p$-divisible groups over odd admissible rings to Dieudonné displays. In the nonodd case there is a $v$-stabilised version of this functor, which will serve as a first step towards the true functor in the next section. We begin with some preparation.

3A. Finiteness over admissible rings. We show that the categories of $p$-divisible groups or Dieudonné displays over an admissible ring $R$ are the direct limit of the corresponding categories over the finitely generated $W\left(R_{\text {red }}\right)$-subalgebras of $R$, with fully faithful transition maps.

Proposition 3.1. Every Dieudonné display over an admissible ring $R$ is defined over a finitely generated $W\left(R_{\mathrm{red}}\right)$-subalgebra of $R$. For an injective homomorphism of admissible rings $R \rightarrow S$ such that $R_{\mathrm{red}} \rightarrow S_{\mathrm{red}}$ is bijective, the base change of Dieudonné displays from $R$ to $S$ is fully faithful.

Proof. For a ring $A$, let $\mathrm{V}(A)$ be the category of projective $A$-modules of finite type. Since the ring $\mathbb{W}(R)$ is the filtered union of $\mathbb{W}\left(R^{\prime}\right)$, where $R^{\prime}$ runs through the finitely generated $W\left(R_{\text {red }}\right)$-subalgebras of $R$, the category $\mathrm{V}(\mathbb{W}(R))$ is equivalent to the direct limit over $R^{\prime}$ of $\mathrm{V}\left(\mathbb{W}\left(R^{\prime}\right)\right)$. Since a Dieudonné display over $R$ can be given by $L, T \in \mathrm{V}(\mathbb{W}(R))$ together with an $f$-linear automorphism $\Psi$ of $L \oplus T$, the first assertion of the proposition follows. Similarly, every homomorphism of Dieudonné displays over $R$ is defined over some finitely generated $R^{\prime}$. Thus for the second assertion we may assume that $\mathcal{N}_{S}^{r}=0$. Let $\bar{S}=S / \mathcal{N}_{S}^{r-1}$ and $\bar{R}=R / R \cap \mathcal{N}_{S}^{r-1}$. Let $R^{\prime \prime} \subseteq S$ be the inverse image of $\bar{R} \subseteq \bar{S}$. By induction on $r$, the base change of Dieudonné displays from $\bar{R}$ to $\bar{S}$ is fully faithful. It follows that the base change from $R^{\prime \prime}$ to $S$ is fully faithful as well. By Corollary 2.10, using trivial divided powers, Dieudonné displays over $R$ or over $R^{\prime \prime}$ are equivalent to Dieudonné displays over $\bar{R}$ together with a lift of the Hodge filtration to $R$ or to $R^{\prime \prime}$, respectively. Since $R \rightarrow R^{\prime \prime}$ is injective, it follows that the base change of Dieudonné displays from $R$ to $R^{\prime \prime}$ is fully faithful.

For the case of $p$-divisible groups we first recall some standard facts.

Lemma 3.2. Let $B \rightarrow A$ be a surjective ring homomorphism with kernel I such that $p I=0$ and $x^{p}=0$ for all $x \in I$. For an affine flat group scheme $H$ over $B$, the kernel of $H(B) \rightarrow H(A)$ is annihilated by $p$. 
Proof. Let $B_{0}=B / p B$ and $H_{0}=H \otimes_{B} B_{0}$. The abelian group $B_{0} \oplus I$ becomes a ring with multiplication $(a \oplus i)\left(a^{\prime} \oplus i^{\prime}\right)=a a^{\prime} \oplus\left(a i^{\prime}+a^{\prime} i+i i^{\prime}\right)$, and one can identify $B \times_{A} B$ with $B \times{ }_{B_{0}}\left(B_{0} \oplus I\right)$. Since the evaluation of affine schemes commutes with fibred products of rings, we obtain an isomorphism of abelian groups

$$
\operatorname{Ker}(H(B) \rightarrow H(A)) \cong \operatorname{Ker}\left(H_{0}\left(B_{0} \oplus I\right) \rightarrow H_{0}\left(B_{0}\right)\right) .
$$

The right-hand side lies in the kernel of the Frobenius $F_{H_{0}}$ of $H_{0}$, which lies in

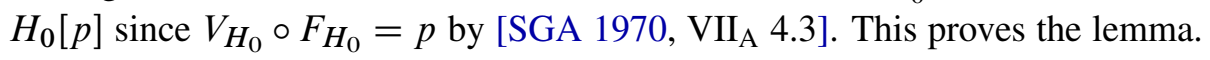

Lemma 3.3. Let $B \rightarrow A$ be a surjective ring homomorphism with kernel I such that $p$ is nilpotent in $B$ and $I$ is a nilideal. For a p-divisible group $G$ over $B$, the homomorphism $G(B) \rightarrow G(A)$ is surjective.

Proof. For a given $x \in G_{n}(A)$, since $G_{n}$ is finitely presented there is a finitely generated ideal $I^{\prime} \subseteq I$ such that $x$ lifts to an element $x^{\prime} \in G_{n}\left(B / I^{\prime}\right)$. Now we can use that $G$ is formally smooth by [Messing 1972, Chapter II, Theorem 3.3.13].

Lemma 3.4. Let $B \rightarrow A$ be a surjective ring homomorphism whose kernel is bounded nilpotent and such that $p$ is nilpotent in $B$. Then there is a number $r$ such that for two p-divisible groups $G$ and $H$ over $B$, the reduction homomorphism $\operatorname{Hom}(G, H) \rightarrow \operatorname{Hom}\left(G_{A}, H_{A}\right)$ is injective with kernel annihilated by $p^{r}$.

Proof. This is an easy consequence of Lemmas 3.2 and 3.3; see the proof of [Katz 1981, Lemma 1.1.3].

Proposition 3.5. Every p-divisible group over an admissible ring $R$ is defined over a finitely generated $W\left(R_{\mathrm{red}}\right)$-subalgebra of $R$. For an injective homomorphism of admissible rings $R \rightarrow S$ such that $R_{\mathrm{red}} \rightarrow S_{\mathrm{red}}$ is bijective, the base change of p-divisible groups from $R$ to $S$ is fully faithful.

Proof. For a $p$-divisible group $G$ over $R$, let $G_{0}=G \otimes_{R} R_{\text {red. }}$. Using Lemma 3.4, we chose $r$ such that for two $p$-divisible groups $G$ and $H$ over $R$, the cokernel of $\operatorname{Hom}(G, H) \rightarrow \operatorname{Hom}\left(G_{0}, H_{0}\right)$ is annihilated by $p^{r}$. Now let $G$ be given, let $G^{\prime \prime}$ be a lift of $G_{0}$ to $W\left(R_{\text {red }}\right)$ and let $G^{\prime}=G^{\prime \prime} \otimes_{W\left(R_{\text {red }}\right)} R$. There are homomorphisms $\varphi$ : $G^{\prime} \rightarrow G$ and $\psi: G \rightarrow G^{\prime}$ which each lift the multiplication $p^{r}: G_{0} \rightarrow G_{0}$. Thus $\varphi \psi$ and $\psi \varphi$ are multiplication by $p^{2 r}$. We obtain an isomorphism $G \cong G^{\prime} / K_{G}$, where $K_{G} \subseteq G^{\prime}$ is a finite locally free group scheme annihilated by $p^{2 r}$; see Lemma 3.6 below. In particular $K_{G}$ is finitely presented, and the first assertion of the proposition follows. To prove the second assertion, we consider two $p$-divisible groups $G$ and $H$ over $R$ and a homomorphism $\varphi_{0}: G_{0} \rightarrow H_{0}$ over $R_{\text {red }}=S_{\text {red }}$. There is a unique lift of $p^{r} \varphi_{0}$ to a homomorphism $\psi: G \rightarrow H$, and there is a lift of $\varphi_{0}$ to $R$ if $\psi$ vanishes on $G\left[p^{r}\right]$. Since $R \rightarrow S$ is injective, this holds if and only if the scalar extension $\psi_{S}$ vanishes on $G_{S}\left[p^{r}\right]$, which is equivalent to the existence of a lift of $\varphi_{0}$ to $S$. 
Lemma 3.6. Let $\varphi: G \rightarrow H$ and $\psi: H \rightarrow G$ be homomorphisms of p-divisible groups over a scheme $S$ with $\varphi \psi=p^{n}$ and $\psi \varphi=p^{n}$. Then $\operatorname{Ker}(\varphi)$ and $\operatorname{Ker}(\psi)$ are finite locally free group schemes.

Proof. Clearly $\operatorname{Ker}(\varphi)$ and $\operatorname{Ker}(\psi)$ are finite group schemes of finite presentation. Thus we may assume that $S=\operatorname{Spec} R$ for a local ring $R$ with residue field $k$. Let $\operatorname{Ker}(\psi)=\operatorname{Spec} A$ and $G_{n}=\operatorname{Spec} B$. Choose elements $a_{1}, \ldots, a_{p^{r}} \in A$ which map to a $k$-basis of $A_{k}$, so they generate $A$ as an $R$-module. We have a surjective homomorphism of fppf sheaves $\varphi: G_{n} \rightarrow \operatorname{Ker}(\psi)$. It follows that $B_{k}$ is a locally free $A_{k}$-module of some rank $p^{s}$, thus a free $A_{k}$-module since $A_{k}$ is finite. Choose $b_{1}, \ldots, b_{p^{s}} \in B$ which map to an $A_{k}$-basis of $B_{k}$. The elements $a_{i} b_{j} \in B$ map to a $k$-basis of $B_{k}$. Since $B$ is a free $R$-module they form an $R$-basis of $B$. It follows that $A$ is free over $R$ with basis $a_{i}$.

3B. Deformation rings. Let $\Lambda \rightarrow K$ be a surjective ring homomorphism with finitely generated kernel $I \subseteq \Lambda$ such that $\Lambda$ is $I$-adically complete. The ring $K$ is not assumed to be a field. Let $\mathrm{Nil}_{\Lambda / K}$ be the category of $\Lambda$-algebras $A$ together with a homomorphism of $\Lambda$-algebras $A \rightarrow K$ with nilpotent kernel. We consider covariant functors

$$
F: \mathrm{Nil}_{\Lambda / K} \rightarrow \text { (sets) }
$$

with the following properties (cf. [Schlessinger 1968]):

(3-1) The set $F(K)$ has precisely one element.

(3-2) For a surjective homomorphism $A_{1} \rightarrow A$ in $\mathrm{Nil}_{\Lambda / K}$, the induced map $F\left(A_{1}\right) \rightarrow F(A)$ is surjective.

(3-3) For each pair of homomorphisms $A_{1} \rightarrow A \leftarrow A_{2}$ in $\mathrm{Nil}_{\Lambda / K}$ such that one of them is surjective, the natural map $F\left(A_{1} \times_{A} A_{2}\right) \rightarrow F\left(A_{1}\right) \times_{F(A)} F\left(A_{2}\right)$ is bijective. Then for each $K$-module $N$ the set $F(K \oplus N)$ is naturally a $K$-module. In particular, $t_{F}=F(K[\varepsilon])$ is a $K$-module, which is called the tangent space of $F$.

(3-4) For each $K$-module $N$ the natural homomorphism of $K$-modules $t_{F} \otimes_{K} N \rightarrow$ $F(K \oplus N)$ is bijective.

(3-5) The $K$-module $t_{F}$ is finitely presented.

The first three conditions imply that the functor $N \mapsto F(K \oplus N)$ preserves exact sequences of $K$-modules. Thus (3-4) is automatic if $N$ is finitely presented. Moreover (3-1)-(3-4) imply that the $K$-module $t_{F}$ is flat, so (3-5) implies that $t_{F}$ is projective.

Proposition 3.7. Assume that $F$ satisfies (3-1)-(3-5). Then $F$ is prorepresented by a complete $\Lambda$-algebra $B$. Let $\tilde{t}$ be a projective $\Lambda$-module of finite type which lifts $t_{F}$. Then $B$ is isomorphic to the complete symmetric algebra $\Lambda \llbracket \tilde{t}^{*} \rrbracket$, where the * means the dual. This is a power series ring over $\Lambda$ if $t_{F}$ is a free $K$-module. 
Proof. The $K$-module $t_{F}$ is projective as explained above. Thus $\tilde{t}$ exists. Let $B=\Lambda \llbracket \tilde{t}^{*} \rrbracket$ and let $\bar{B}=K \oplus t_{F}^{*}$. We have an obvious projection $B \rightarrow \bar{B}$. Let $\bar{\xi} \in F(\bar{B})=t_{F} \otimes t_{F}^{*}=\operatorname{End}\left(t_{F}\right)$ correspond to the identity of $t_{F}$ and let $\xi \in F(B)$ be a lift of $\bar{\xi}$. We claim that the induced homomorphism of functors $\xi: B \rightarrow F$ is bijective. Note that the functor $B$ satisfies (3-1)-(3-5). By induction it suffices to show that if $A \rightarrow \bar{A}$ is a surjection in $\mathrm{Nil}_{\Lambda / K}$ whose kernel $N$ is a $K$-module of square zero and if $B(\bar{A}) \rightarrow F(\bar{A})$ is bijective, then $B(A) \rightarrow F(A)$ is bijective as well. We have a natural isomorphism $A \times_{\bar{A}} A \cong A \times_{K}(K \oplus N)$. It follows that the fibres of $B(A) \rightarrow B(\bar{A})$ and the fibres of $F(A) \rightarrow F(\bar{A})$ are principal homogeneous sets under the $K$-modules $B(K \oplus N)$ and $F(K \oplus N)$, respectively. The homomorphism $t_{B} \rightarrow t_{F}$ induced by $\xi$ is bijective by construction, so $B(K \oplus N) \rightarrow F(K \oplus N)$ is bijective, and the proposition follows.

Corollary 3.8. A homomorphism of functors which satisfy (3-1)-(3-5) is an isomorphism if and only if it induces an isomorphism on the tangent spaces.

Remark 3.9. Let $\Lambda^{\prime} \rightarrow K^{\prime}$ be another pair as above and let $g: \Lambda^{\prime} \rightarrow \Lambda$ be a ring homomorphism which induces a homomorphism $\bar{g}: K^{\prime} \rightarrow K$. For given functors $F$ on $\mathrm{Nil}_{\Lambda / K}$ and $F^{\prime}$ on $\mathrm{Nil}_{\Lambda^{\prime} / K^{\prime}}$, a homomorphism $h: F \rightarrow F^{\prime}$ over $g$ is a compatible system of maps

$$
h(A): F(A) \rightarrow F^{\prime}\left(A \times_{K} K^{\prime}\right)
$$

for $A$ in $\mathrm{Nil}_{\Lambda / K}$; here $A \times_{K} K^{\prime}$ is naturally an object of $\mathrm{Nil}_{\Lambda^{\prime} / K^{\prime}}$. If $F$ and $F^{\prime}$ satisfy (3-1)-(3-5) and if $B$ and $B^{\prime}$ are the complete algebras which prorepresent $F$ and $F^{\prime}$, respectively, then $h$ corresponds to a homomorphism $B^{\prime} \rightarrow B$ compatible with $g$ and $\bar{g}$. If $h(A)$ is bijective for all $A$, the induced homomorphism $B^{\prime} \hat{\otimes}_{\Lambda^{\prime}} \Lambda \rightarrow B$ is an isomorphism.

Definition 3.10. Assume that $p$ is nilpotent in $K=\Lambda / I$ as above. For a $p$-divisible group $G$ over $K$ let

$$
\operatorname{Def}_{G}: \mathrm{Nil}_{\Lambda / K} \rightarrow \text { (sets) }
$$

be the deformation functor of $G$. This means that $\operatorname{Def}_{G}(A)$ is the set of isomorphism classes of $p$-divisible groups $G^{\prime}$ over $A$ together with an isomorphism $G^{\prime} \otimes_{A} K \cong G$. Let $t_{G}=\operatorname{Lie}\left(G^{\vee}\right) \otimes_{K} \operatorname{Lie}(G)$.

Proposition 3.11. The functor $\operatorname{Def}_{G}$ is prorepresented by a complete $\Lambda$-algebra $B$. Explicitly, if $\tilde{t}$ is a projective $\Lambda$-module which lifts $t_{G}$, then $B$ is isomorphic to the complete symmetric algebra $\Lambda \llbracket \tilde{t}^{*} \rrbracket$.

We note that Lemma 2.15 gives a universal $p$-divisible group over $B$.

Proof. The functor $\operatorname{Def}_{G}$ satisfies (3-1)-(3-5) with tangent space $t_{G}$ because for a surjective homomorphism $A^{\prime} \rightarrow A$ in $\operatorname{Nil}_{\Lambda / K}$ whose kernel $N$ is a $K$-module 
of square zero and for $H \in \operatorname{Def}_{G}(A)$, the set of lifts of $H$ to $A^{\prime}$ is a principally homogeneous set under the $K$-module $t_{G} \otimes_{K} N$ by [Messing 1972].

Remark 3.12. Let $g: \Lambda^{\prime} \rightarrow \Lambda$ over $\bar{g}: K^{\prime} \rightarrow K$ be as in Remark 3.9, such that $p$ is nilpotent in $K^{\prime}$. Let $G$ over $K$ be the base change of a $p$-divisible group $G^{\prime}$ over $K^{\prime}$. For $A$ in $\mathrm{Nil}_{\Lambda / K}$ we have a natural map

$$
\operatorname{Def}_{G^{\prime}}\left(A \times_{K} K^{\prime}\right) \rightarrow \operatorname{Def}_{G}(A) .
$$

This map is bijective, and its inverse is a homomorphism $\operatorname{Def}_{G} \rightarrow \operatorname{Def}_{G^{\prime}}$ over $g$ in the sense of Remark 3.9. If $B$ and $B^{\prime}$ prorepresent $\operatorname{Def}_{G}$ and $\operatorname{Def}_{G^{\prime}}$, respectively, we get an isomorphism $B^{\prime} \hat{\otimes}_{\Lambda^{\prime}} \Lambda \cong B$.

Definition 3.13. Assume that $K=\Lambda / I$ is an admissible ring. For a Dieudonné display $\mathscr{P}=\left(P, Q, F, F_{1}\right)$ over $K$, we denote by

$$
\operatorname{Def}_{\mathscr{P}}: \operatorname{Nil}_{\Lambda / K} \rightarrow \text { (sets) }
$$

the deformation functor of $\mathscr{P}$. Let $t_{\mathscr{P}}=\operatorname{Hom}\left(Q / \square_{K} P, P / Q\right)$.

We are mainly interested in the case where $K$ is perfect and $\Lambda=W(K)$. Then Dieudonné displays over $K$ are displays because $\mathbb{W}(K)=W(K)$.

Proposition 3.14. The functor $\operatorname{Def}_{\mathscr{P}}$ is prorepresented by a complete $\Lambda$-algebra $B$. Explicitly, if $\tilde{t}$ is a projective $\Lambda$-module which lifts $t_{\mathscr{P}}$, then $B$ is isomorphic to the complete symmetric algebra $\Lambda \llbracket \tilde{t}^{*} \rrbracket$.

We note that Lemma 2.16 gives a universal Dieudonné display over $B$.

Proof. The functor Def $\mathscr{P}$ satisfies (3-1)-(3-5) with tangent space $t_{\mathscr{P}}$ because for a surjective homomorphism $A^{\prime} \rightarrow A$ in $\operatorname{Nil}_{\Lambda / K}$ whose kernel $N$ is a $K$-module of square zero and for $\mathscr{P}^{\prime} \in \operatorname{Def}_{\mathscr{P}}(A)$, the set of lifts of $\mathscr{P}^{\prime}$ to $A^{\prime}$ is a principally homogeneous set under the $K$-module $t_{\mathscr{P}} \otimes_{K} N$ by Corollary 2.10.

Remark 3.15. Let $g: \Lambda^{\prime} \rightarrow \Lambda$ over $\bar{g}: K^{\prime} \rightarrow K$ be as in Remark 3.9, such that $K$ and $K^{\prime}$ are admissible rings. Assume that $\mathscr{P}$ is the base change of a Dieudonné display $\mathscr{P}^{\prime}$ over $K^{\prime}$. If $B$ and $B^{\prime}$ represent $\operatorname{Def}_{\mathscr{P}}$ and $\operatorname{Def}_{\mathscr{P}^{\prime}}$, respectively, then $B^{\prime} \hat{\otimes}_{\Lambda^{\prime}} \Lambda \cong B$. This is analogous to Remark 3.12.

3C. Crystals and frames. Let $\mathcal{F}=\left(S, I, R, \sigma, \sigma_{1}\right)$ be a frame as in Section 2A, such that $S$ and $R$ are $p$-adically complete, $S$ has no $p$-torsion, $I$ carries divided powers, and $\sigma=p \sigma_{1}$ on $I$. Thus $(S, \sigma)$ is a frame for each $R / p^{n} R$ in the sense of [Zink 2001b]. By a well-known construction, the crystalline Dieudonné functor allows us to associate to a $p$-divisible group over $R$ an $\mathcal{F}$-window; this is explained in the proof of [Zink 2001b, Theorem 1.6] for the Dieudonné crystal of a nilpotent display, and in [Kisin 2006; 2009] for $p$-divisible groups.

The construction goes as follows. First, one can define a filtered $F-V$-module; here it is not necessary to assume that $S$ has no $p$-torsion. 
Construction 3.16. Let $\mathcal{F}=\left(S, I, R, \sigma, \sigma_{1}\right)$ be a frame such that $S$ and $R$ are $p$-adically complete, $I$ is equipped with divided powers $\delta$ which are compatible with the canonical divided powers of $p$, and $\sigma=p \sigma_{1}$ on $I$. Let $\delta^{\prime}$ be the divided powers on $I^{\prime}=I+p S$ which extend $\delta$ and the canonical divided powers of $p$. We assume that $\sigma$ preserves $\delta^{\prime}$, which is automatic if $S$ has no $p$-torsion. Then one can define a functor

$\Phi^{o}:(p$-divisible groups over $R) \rightarrow($ filtered $F-V$-modules over $\mathcal{F})$,

$$
G \mapsto\left(P, Q, F^{\#}, V^{\sharp}\right)
$$

as follows. Let $R_{0}=R / p R$ and let $\sigma_{0}$ be its Frobenius endomorphism. For a given $p$-divisible group $G$ over $R$ put $P=\mathbb{D}(G)_{S / R}=\mathbb{D}\left(G_{0}\right)_{S / R_{0}}$, where $\mathbb{D}(G)$ is the covariant ${ }^{2}$ Dieudonné crystal of $G$, and let $Q$ be the kernel of the natural map $P \rightarrow \operatorname{Lie}(G)$. Since $\sigma$ preserves $\delta^{\prime}$, there is a natural isomorphism

$$
P^{(\sigma)} \cong \mathbb{D}\left(\sigma_{0}^{*} G_{0}\right)_{S / R_{0}} .
$$

Thus we can define $V^{\sharp}: P \rightarrow P^{(\sigma)}$ to be induced by the Frobenius $F: G_{0} \rightarrow \sigma_{0}^{*} G_{0}$ and $F^{\sharp}: P^{(\sigma)} \rightarrow P$ to be induced by the Verschiebung $V: \sigma_{0}^{*} G_{0} \rightarrow G_{0}$.

In the second step one associates $F_{1}$.

Proposition 3.17. Let $\mathcal{F}$ be a frame as in the beginning of Section 3C. For a $p$ divisible group $G$ over $R$ let $\Phi^{o}(G)=\left(P, Q, F^{\sharp}, V^{\sharp}\right)$ be the filtered $F$-V-module over $\mathcal{F}$ given by Construction 3.16. There is a unique $F_{1}: Q \rightarrow P$ such that $\left(P, Q, F, F_{1}\right)$ is an $\mathcal{F}$-window, and it gives back $V^{\sharp}$ by the functor of Lemma 2.3. Proof. We have functors $\left(P, Q, F, F_{1}\right) \mapsto\left(P, Q, F^{\sharp}, V^{\sharp}\right) \mapsto\left(P, Q, F^{\sharp}\right)$, which are fully faithful; see Lemma 2.3. Thus we have to show that $F(Q)$ lies in $p P$ so that $F_{1}=p^{-1} F$ is well-defined, that $F_{1}(Q)$ generates $P$, and that the pair $(P, Q)$ admits a normal decomposition. Since $R$ and $S$ are $p$-adically complete and since the kernel of $S / p S \rightarrow R / p R$ is a nilideal due to its divided powers, all projective $R$-modules of finite type lift to $S$. Thus a normal decomposition exists. The existence of $F_{1}$ and the surjectivity of its linearisation are proved in [Kisin 2006, Lemma A.2] if $S$ is local with perfect residue field, but the proof can be easily adapted to the general case. To prove surjectivity, for each maximal ideal of $S$, which necessarily comes from a maximal ideal $\mathfrak{m}$ of $R$, we choose an embedding of $R / \mathfrak{m}$ into a perfect field $k$. There is a ring homomorphism $\alpha: S \rightarrow W(k)$ which lifts $R \rightarrow k$ such that $f \alpha=\alpha \sigma$; it can be constructed as $S \rightarrow W(S) \rightarrow W(k)$. Then $\alpha$ is a homomorphism of frames $\mathcal{F} \rightarrow \mathscr{W}_{k}$, and the assertion is reduced to the case of $\mathscr{W}_{k}$, which is classical.

2 This differs from the notation of [Berthelot et al. 1982], where $\mathbb{D}(G)$ is contravariant. One can switch between the covariant and contravariant crystals by passing to the dual of $G$ or of $\mathbb{D}(G)$, which amount to the same thing by the crystalline duality theorem [Berthelot et al. 1982, 5.3]. 
Remark 3.18. The surjectivity of $F_{1}$ in the proof of Proposition 3.17 can also be deduced from the crystalline duality theorem. Let $P=L \oplus T$ be a normal decomposition and let $\Psi: P \rightarrow P$ be given by $F_{1}$ on $L$ and by $F$ on $T$. We have to show that the linearisation $\Psi^{\sharp}: P^{(\sigma)} \rightarrow P$ is an isomorphism. Let $\left(P^{\prime}, Q^{\prime}, F^{\prime}, F_{1}^{\prime}\right)$ be the quadruple associated to the Cartier dual $G^{\vee}$. The duality theorem gives a perfect pairing $P \times P^{\prime} \rightarrow S$ such that $\left\langle F(x), F^{\prime}\left(x^{\prime}\right)\right\rangle=p \sigma\left\langle x, x^{\prime}\right\rangle$. It follows that $\left\langle F(x), F_{1}^{\prime}\left(x^{\prime}\right)\right\rangle=\sigma\left\langle x, x^{\prime}\right\rangle$ and $\left\langle F_{1}(x), F^{\prime}\left(x^{\prime}\right)\right\rangle=\sigma\left\langle x, x^{\prime}\right\rangle$ whenever this makes sense. The unique decomposition $P^{\prime}=L^{\prime} \oplus T^{\prime}$ with $\left\langle L, L^{\prime}\right\rangle=0=\left\langle T, T^{\prime}\right\rangle$ is a normal decomposition of $P^{\prime}$, and the dual of the associated $\Psi^{\prime \sharp}$ is an inverse of $\Psi^{\sharp}$.

3D. The Dieudonné display associated to a p-divisible group. For an admissible ring $R$ with $p \geq 3$, we consider the Dieudonné frame $\mathscr{D}_{R}$ defined in Lemma 2.5. The ring $\mathbb{W}(R)$ is $p$-adically complete by the remark preceding Proposition 1.14. By Lemma 1.17 the ideal $\square_{R}$ carries natural divided powers compatible with the canonical divided powers of $p$, and the induced divided powers on the kernel of $\mathbb{W}(R) \rightarrow R / p R$ are preserved by the Frobenius. Thus Construction 3.16 gives a functor

$\Phi_{R}^{o}:(p$-divisible groups over $R) \rightarrow\left(\right.$ filtered $F-V$-modules over $\left.\mathscr{D}_{R}\right)$

which is compatible with base change in $R$.

Theorem 3.19. For each admissible ring $R$ with $p \geq 3$, there is a unique functor

$\Phi_{R}:($-divisible groups over $R) \rightarrow($ Dieudonné displays over $R)$

which is compatible with base change in $R$ such that the filtered $F$ - $V$-module over $\mathscr{D}_{R}$ associated to $\Phi_{R}(G)$ is equal to $\Phi_{R}^{o}(G)$. In particular there is a natural isomorphism $\operatorname{Lie}(G) \cong \operatorname{Lie}\left(\Phi_{R}(G)\right)$.

Proof. Clearly $\Phi_{R}^{o}(G)=\left(P, Q, F^{\#}, V^{\sharp}\right)$ is functorial in $R$ and $G$. We have to show that there is a unique operator $F_{1}: Q \rightarrow P$ which is functorial in $R$ and $G$ such that $\Phi_{R}(G)=\left(P, Q, F, F_{1}\right)$ is a Dieudonné display over $R$.

Let $K=R_{\text {red }}$ and $\Lambda=W(K)$. Let $\bar{G}=G \otimes_{R} K$ and let $B$ be the complete $\Lambda$-algebra which prorepresents the functor $\operatorname{Def}_{\bar{G}}$ on $\mathrm{Nil}_{\Lambda / K}$; see Proposition 3.11. Let $\mathscr{G}$ be the universal deformation of $G$ over $B$. If $I$ denotes the kernel of $B \rightarrow K$, we can define

$$
\Phi_{B}^{o}(\mathscr{G})=\lim _{n} \Phi_{B / I^{n}}^{o}\left(\mathscr{G} \otimes_{B} B / I^{n}\right)
$$

On the other hand, the $\operatorname{ring} \mathbb{W}(B)$ is $p$-adically complete by Proposition 1.14. Therefore we can also define $\Phi_{B}^{o}(\mathscr{G})$ be a direct application of Construction 3.16, and this agrees with the limit definition. The ring $\mathbb{W}(B)$ has no $p$-torsion because 
$B$ has no $p$-torsion. Thus by Proposition 3.17 there is a unique operator $F_{1}$ which makes $\Phi_{B}^{o}(\mathscr{G})$ into a Dieudonné display $\Phi_{B}(\mathscr{G})$ over $B$.

By Proposition 3.5 there is a unique homomorphism $B \rightarrow R$ of augmented algebras such that $G=\mathscr{G} \otimes_{B} R$ as deformations of $\bar{G}$. Necessarily we define $\Phi_{R}(G)$ as the base change of $\Phi_{B}(\mathscr{G})$ under $B \rightarrow R$. It remains to show that $\Phi_{R}(G)$ is functorial in $R$ and $G$.

Assume that $G$ is the base change of a $p$-divisible group $G^{\prime}$ over $R^{\prime}$ under a homomorphism of admissible rings $R^{\prime} \rightarrow R$. Let $K^{\prime}, \Lambda^{\prime}, \bar{G}^{\prime}, B^{\prime}, \mathscr{G}^{\prime}$ have the obvious meaning. We have a natural homomorphism of $W\left(K^{\prime}\right)$-algebras $B^{\prime} \rightarrow B$ together with an isomorphism $\mathscr{G}^{\prime} \otimes_{B^{\prime}} B \cong \mathscr{G}$; see Remark 3.12. By the uniqueness of $F_{1}$ over $B$, we see that $\Phi_{B}(\mathscr{G})$ coincides with the base change of $\Phi_{B^{\prime}}\left(\mathscr{G}^{\prime}\right)$. It follows that $\Phi_{R}(G)$ is the base change of $\Phi_{R^{\prime}}\left(G^{\prime}\right)$.

Assume that $u: G \rightarrow G_{1}$ is a homomorphism of $p$-divisible groups over $R$. Let $\bar{G}_{1}, B_{1}, \mathscr{G}_{1}$ have the obvious meaning. We have to show that $\Phi_{R}^{o}(u)$ commutes with $F_{1}$. We may assume that $u$ is an isomorphism because otherwise one can pass to the automorphism $\left(\begin{array}{ll}1 & 0 \\ u & 1\end{array}\right)$ of $G \oplus G_{1}$. This reasoning uses that the natural isomorphism $\Phi_{R}^{o}\left(G \oplus G_{1}\right)=\Phi_{R}^{o}(G) \oplus \Phi_{R}^{o}\left(G_{1}\right)$ preserves the operators $F_{1}$ defined on the three modules, which follows from the uniqueness of $F_{1}$ over the ring which prorepresents $\operatorname{Def}_{\bar{G}} \times \operatorname{Def}_{\bar{G}_{1}}$. An isomorphism $u: G \rightarrow G_{1}$ induces an isomorphism $\bar{u}: \bar{G} \cong \bar{G}_{1}$, which gives an isomorphism $B \cong B_{1}$ together with an isomorphism $\tilde{u}: \mathscr{G} \otimes_{B} B_{1} \cong \mathscr{G}_{1}$ that lifts $\bar{u}$. By the uniqueness of $F_{1}$ over $B_{1}$ it follows that $\Phi_{B_{1}}^{o}(\tilde{u})$ preserves $F_{1}$. Since $u$ is the base change of $\tilde{u}$ by the homomorphism $B_{1} \rightarrow R$ defined by $G_{1}$, it follows that $\Phi_{R}^{o}(u)$ preserves $F_{1}$ as well.

In order to analyse the action of the functors $\Phi_{R}$ on infinitesimal deformations, we need the following extension of Theorem 3.19. Let $\left(R^{\prime} \rightarrow R, \delta\right)$ be a divided power extension of admissible rings with $p \geq 3$ which is compatible with the canonical divided powers of $p$. Again, the ring $\mathbb{W}\left(R^{\prime} / R\right)$ is $p$-adically complete, and $\mathbb{R}_{R^{\prime} / R}$ carries natural divided powers compatible with the canonical divided powers of $p$ such that $f$ preserves their extension to the kernel of $\mathbb{W}\left(R^{\prime} / R\right) \rightarrow R / p R$. Thus Construction 3.16 gives a functor

$\Phi_{R^{\prime} / R}^{o}:(p$-divisible groups over $R) \rightarrow\left(\right.$ filtered $F$ - $V$-modules over $\left.\mathscr{D}_{R^{\prime} / R}\right)$

which is compatible with base change in the triple $\left(R^{\prime} \rightarrow R, \delta\right)$.

Theorem 3.20. Assume that $p \geq 3$. For each divided power extension of admissible rings $\left(R^{\prime} \rightarrow R, \delta\right)$ compatible with the canonical divided powers of $p$, there is a unique functor

$\Phi_{R^{\prime} / R}:($ p-divisible groups over $R) \rightarrow\left(\right.$ Dieudonné displays for $\left.R^{\prime} / R\right)$ 
which is compatible with base change in the triple $\left(R^{\prime} \rightarrow R, \delta\right)$ such that the filtered $F$-V-module over $\mathscr{D}_{R^{\prime} / R}$ associated to $\Phi_{R^{\prime} / R}(G)$ is equal to $\Phi_{R^{\prime} / R}^{o}(G)$.

Proof. For a given $p$-divisible group $G$ over $R$ we choose a lift to a $p$-divisible group $G^{\prime}$ over $R^{\prime}$, which exists by [Illusie 1985, Théorème 4.4]. The Dieudonné display $\Phi_{R^{\prime}}\left(G^{\prime}\right)$ is well-defined by Theorem 3.19, and necessarily $\Phi_{R^{\prime} / R}(G)$ is defined as the base change of $\Phi_{R^{\prime}}\left(G^{\prime}\right)$ by the frame homomorphism $\mathscr{D}_{R^{\prime}} \rightarrow \mathscr{D}_{R^{\prime} / R}$. We have to show that the operator $F_{1}$ on $\Phi_{R^{\prime} / R}^{o}(G)$ defined in this way does not depend on the choice of $G^{\prime}$. If this is proved it follows easily that $\Phi_{R^{\prime} / R}(G)$ is functorial in $G$ and in $\left(R^{\prime} \rightarrow R, \delta\right)$; here, instead of arbitrary homomorphisms of $p$-divisible groups, it suffices to treat isomorphisms.

Let $K, \Lambda, \bar{G}, B, \mathscr{G}$ be as in the proof of Theorem 3.19. We have an isomorphism $B \cong \Lambda \llbracket t \rrbracket$ for a finitely generated projective $\Lambda$-module $t$. Let $C=B \hat{\otimes}_{\Lambda} B$. The automorphism $\tau=\left(\begin{array}{ll}1 & 1 \\ 0 & 1\end{array}\right)$ of $t \oplus t$ defines an isomorphism

$$
C=\Lambda \llbracket t \oplus t \rrbracket \stackrel{\tau}{\longrightarrow} \Lambda \llbracket t \oplus t \rrbracket=B \llbracket t_{B} \rrbracket
$$

under which the multiplication homomorphism $\mu: C \rightarrow B$ corresponds to the augmentation $B \llbracket t_{B} \rrbracket \rightarrow B$ defined by $t_{B} \mapsto 0$. Let $I$ be the kernel of $B \rightarrow K$, let $S$ be the divided power envelope of the ideal $t_{B} B \llbracket t_{B} \rrbracket \subseteq B \llbracket t_{B} \rrbracket$, and let $C^{\prime}$ be the $I$-adic completion of $S$. By Lemma 1.13, $\mu$ extends to a divided power extension of admissible topological rings $\mu^{\prime}: C^{\prime} \rightarrow B$ which is topologically compatible with the canonical divided powers of $p .^{3}$

Assume that $G_{1}$ and $G_{2}$ are two lifts of $G$ to $p$-divisible groups over $R^{\prime}$. Let $\mathscr{G}_{1}$ and $\mathscr{G}_{2}$ be the $p$-divisible groups over $C$ which are the base change of $\mathscr{G}$ by the two natural homomorphisms $B \rightarrow C$. By Proposition 3.5 there are well-defined homomorphisms $\bar{\alpha}: B \rightarrow R$ and $\alpha: C \rightarrow R^{\prime}$ such that $G=\mathscr{G} \otimes_{B, \bar{\alpha}} R$ and $G_{i}=\mathscr{G}_{i} \otimes_{C, \alpha} R^{\prime}$ as deformations of $\bar{G}$. We have the commutative diagram of rings

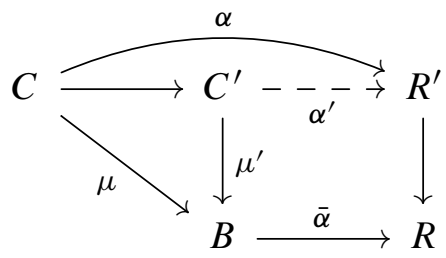

where $\alpha^{\prime}$ is constructed as follows. There is a unique homomorphism $\alpha^{\prime \prime}: S \rightarrow R^{\prime}$ which extends $\alpha$ and which commutes with the divided powers on the kernel of $S \rightarrow B$ and of $R^{\prime} \rightarrow R$. Each of the two homomorphisms $B \rightarrow C \rightarrow R^{\prime}$ factors over $B / I^{n}$ for some $n$. Thus $\alpha^{\prime \prime}$ induces a homomorphism $S / I^{n} S \rightarrow R^{\prime}$, which

\footnotetext{
${ }^{3}$ The construction of $C^{\prime}$ seems to depend on choosing one of the two natural maps $B \rightarrow C$, but actually it is independent of the choice as the $I$-adic topologies defined on $S$ by these two maps coincide.
} 
gives the required $\alpha^{\prime}$. We obtain the following commutative diagram of frames, where $\iota$ is given by $C \rightarrow C^{\prime}$, and $\iota^{\prime}$ is given by the identity of $R^{\prime}$ :

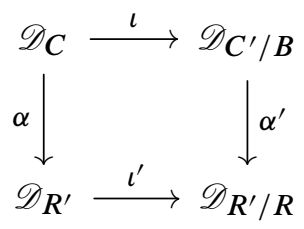

We have to show that the isomorphism of filtered $F$ - $V$-modules over $\mathscr{D}_{R^{\prime} / R}$

$$
\iota_{*}^{\prime}\left(\Phi_{R^{\prime}}^{o}\left(G_{1}\right)\right) \cong \Phi_{R^{\prime} / R}^{o}(G) \cong \iota_{*}^{\prime}\left(\Phi_{R^{\prime}}^{o}\left(G_{2}\right)\right)
$$

commutes with the operator $F_{1}$ defined on the outer terms by the functor $\Phi_{R^{\prime}}$. The construction of $\Phi^{o}$ can be extended to topological divided power extensions of admissible topological rings by passing to the projective limit. Then (3-6) arises by $\alpha_{*}^{\prime}$ from the natural isomorphism of filtered $F-V$-modules over $\mathscr{D}_{C^{\prime} / B}$

$$
\iota_{*}\left(\Phi_{C}^{o}\left(\mathscr{G}_{1}\right)\right) \cong \Phi_{C^{\prime} / B}^{o}(\mathscr{G}) \cong \iota_{*}\left(\Phi_{C}^{o}\left(\mathscr{G}_{2}\right)\right) .
$$

Since $\alpha_{*}$ preserves $F_{1}$ it suffices to show that (3-7) commutes with the operators $F_{1}$ defined on the outer terms by the functor $\Phi_{C}$. This follows from the relation $p F_{1}=F$ because $\mathbb{W}\left(C^{\prime} / B\right)$ has no $p$-torsion by Lemma 1.13 .

Corollary 3.21. Assume that $p \geq 3$. For a $p$-divisible group $G$ over an admissible ring $R$ with associated Dieudonné display $\mathscr{P}=\Phi_{R}(G)$, there is a natural isomorphism of crystals on $\operatorname{Cris}_{\mathrm{adm}}\left(R / \mathbb{Z}_{p}\right)$

$$
\mathbb{D}(G) \cong \mathbb{D}(\mathscr{P})
$$

which is compatible with the natural isomorphism $\operatorname{Lie}(G) \cong \operatorname{Lie}(\mathscr{P})$.

The category Cris adm and the crystal $\mathbb{D}(\mathscr{P})$ were defined in Section $2 \mathrm{~F}$.

Proof. Let $\left(R^{\prime} \rightarrow R, \gamma\right)$ be a divided power extension of admissible rings with $p \geq 3$ compatible with the canonical divided powers of $p$. The Dieudonne display $\Phi_{R^{\prime} / R}(G)$ given by Theorem 3.20 is the unique lift of $\mathscr{P}$ under the crystalline frame homomorphism $\mathscr{D}_{R^{\prime} / R} \rightarrow \mathscr{D}_{R}$. By the construction of the underlying filtered $F$ - $V$-module $\Phi_{R^{\prime} / R}^{o}(G)$ and by the definition of the crystal $\mathbb{R}(\mathscr{P})$ in Section $2 \mathrm{~F}$ we obtain a natural isomorphism of $\mathbb{W}\left(R^{\prime} / R\right)$-modules

$$
\mathbb{D}(G)_{\mathbb{W}\left(R^{\prime}\right) / R} \cong \mathbb{K}(\mathscr{P})_{R^{\prime} / R}
$$

The tensor product with the projection $\mathbb{W}\left(R^{\prime} / R\right) \rightarrow R^{\prime}$, which is a homomorphism of divided power extensions of $R$, gives a natural isomorphism of $R^{\prime}$ modules $\mathbb{D}(G)_{R^{\prime} / R} \cong \mathbb{D}(\mathscr{P})_{R^{\prime} / R}$ which is compatible with the natural isomorphism $\operatorname{Lie}(G) \cong \operatorname{Lie}(\mathscr{P})$. 
Now Theorem B for odd primes can be deduced quite formally:

Corollary 3.22. Assume that $p \geq 3$. For a $p$-divisible group $G$ over an admissible ring $R$ with associated Dieudonné display $\mathscr{P}=\Phi_{R}(G)$, there is a natural isomorphism of crystals on $\mathrm{Cris}_{\mathrm{adm}}(R)$

$$
\mathbb{D}(G) \cong \mathbb{D}(\mathscr{P})
$$

which is compatible with the natural isomorphism $\operatorname{Lie}(G) \cong \operatorname{Lie}(\mathscr{P})$.

Here the covariant Dieudonné crystal $\mathbb{D}(G)$ can be defined for divided power extensions that are not necessarily compatible with the canonical divided powers of $p$ by [Mazur and Messing 1974, Chapter II §9]; see also [Berthelot et al. 1982, §1.4]. Proof. Let $\mathbb{D}^{\prime}(G)=\mathbb{D}\left(\Phi_{R}(G)\right)$. Consider a divided power extension $R^{\prime} \rightarrow R$ of admissible rings which need not be compatible with the canonical divided powers of $p$. We claim that for two lifts $G_{1}$ and $G_{2}$ of $G$ to $R^{\prime}$ the following diagram of natural isomorphisms commutes:

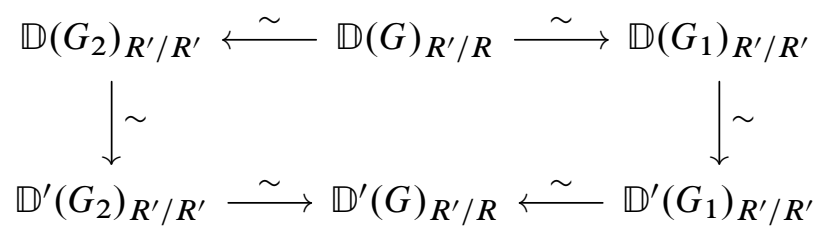

This gives a well-defined isomorphism $\alpha(G): \mathbb{D}(G)_{R^{\prime} / R} \cong \mathbb{D}^{\prime}(G)_{R^{\prime} / R}$. It is easy to see that $\alpha(G)$ is compatible with the natural isomorphism $\operatorname{Lie}(G) \cong \operatorname{Lie}(\mathscr{P})$, that $\alpha(G)$ is functorial in the divided power extension $R^{\prime} \rightarrow R$ and that $\alpha(G \oplus H)=$ $\alpha(G) \oplus \alpha(H)$. In order to show that $\alpha$ is functorial in $G$ it suffices to consider isomorphisms. So let $u: G \rightarrow H$ be an isomorphism of $p$-divisible groups over $R$. We can choose lifts $G_{1}$ of $G$ and $H_{1}$ of $H$ to $R^{\prime}$ such that $u$ extends to $\tilde{u}: G_{1} \rightarrow H_{1}$. Then the following diagram shows that $\alpha$ commutes with $u$ :

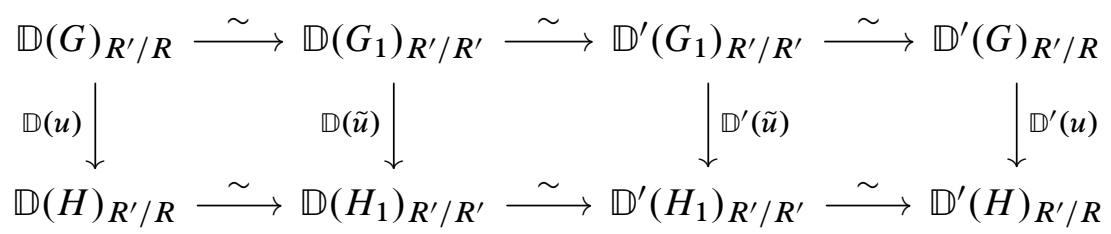

It remains to show that (3-8) commutes. Let $K, \Lambda, \bar{G}, B$ be as in the proof of Theorem 3.19. Let $C=B \widehat{\otimes}_{\Lambda} B$ and $C^{\prime}$ be as in the proof of Theorem 3.20 so that the multiplication homomorphism $\mu: C \rightarrow B$ extends to a topological divided power extension $\mu^{\prime}: C^{\prime} \rightarrow B$ of admissible topological rings without $p$-torsion which is topologically compatible with the canonical divided powers of $p$. We have homomorphisms $B \rightarrow R$ defined by $G$ and $C \rightarrow R^{\prime}$ defined by $\left(G_{1}, G_{2}\right)$, 
which extend to a homomorphism of divided power extensions from $\left(C^{\prime} \rightarrow B\right)$ to $\left(R^{\prime} \rightarrow R\right)$. Thus (3-8) is the base change of a similar diagram for $\left(C^{\prime} \rightarrow B\right)$, which commutes by Corollary 3.21.

3E. $\boldsymbol{A}$ v-stabilised variant. Let $R$ be an admissible ring with $p=2$. The $v$ stabilised Zink ring $\mathbb{W}^{+}(R)$ considered in Section 1D and in Section 2E is 2-adically complete, and its ideal $\square_{R}^{+}$carries natural divided powers which are compatible with the canonical divided powers of 2 . The proof of Theorem 3.19 with $\mathbb{W}^{+}$in place of W shows the following:

Proposition 3.23. For each admissible ring $R$ with $p=2$ there is a unique functor

$$
\Phi_{R}^{+}:(2 \text {-divisible groups over } R) \rightarrow\left(\mathscr{D}_{R}^{+} \text {-windows }\right)
$$

which is compatible with base change in $R$ such that the filtered $F$-V-module over $\mathscr{D}_{R}^{+}$associated to $\Phi_{R}^{+}(G)$ is given by Construction 3.16 .

Corollary 3.24. For each admissible ring $R$ with $p=2$ and $2 R=0$ there is a unique functor

$$
\left.\Phi_{R}: \text { (2-divisible groups over } R\right) \rightarrow(\text { Dieudonné displays over } R)
$$

which is compatible with base change in $R$ such that the filtered $F-V$-module over $\mathscr{D}_{R}$ associated to $\Phi_{R}(G)$ is given by Construction 3.16.

Proof. Proposition 3.23 gives the functors $\Phi_{R}$ since $\mathscr{D}_{R}^{+}=\mathscr{D}_{R}$ when $2 R=0$. The uniqueness follows as in the proof of Theorem 3.19, using $B / 2 B$ instead of $B$.

Let $\left(R^{\prime} \rightarrow R, \delta\right)$ be a divided power extension of admissible rings with $p=2$ which is compatible with the canonical divided powers of 2 . The ring $\mathbb{W}^{+}\left(R^{\prime} / R\right)$ is 2-adically complete, and its ideal $\square_{R^{\prime} / R}^{+}$carries natural divided powers compatible with the canonical divided powers of 2 . The proof of Theorem 3.20 with $\mathbb{W}^{+}$in place of $\mathbb{W}$ gives the following:

Proposition 3.25. For each divided power extension of admissible rings $\left(R^{\prime} \rightarrow R, \delta\right)$ with $p=2$ such that $\delta$ is compatible with the canonical divided powers of 2 there is a unique functor

$$
\Phi_{R^{\prime} / R}^{+}:(2-\text { divisible groups over } R) \rightarrow\left(\mathscr{D}_{\left.R^{\prime} / R^{-w i n d o w s}\right)}^{+}\right.
$$

which is functorial in the triple $\left(R^{\prime} \rightarrow R, \delta\right)$ such that the filtered $F-V$-module over $\mathscr{D}_{R^{\prime} / R}^{+}$associated to $\Phi_{R^{\prime} / R}^{+}(G)$ is given by Construction 3.16 .

The proof of Corollary 3.21 then shows the following: 
Corollary 3.26. Assume that $p=2$. For a 2-divisible group $G$ over an admissible ring $R$ with associated $v$-stabilised Dieudonné display $\mathscr{P}^{+}=\Phi_{R}^{+}(G)$ there is a natural isomorphism of crystals on $\mathrm{Cris}_{\mathrm{adm}}\left(R / \mathbb{Z}_{2}\right)$

$$
\mathbb{D}(G) \cong \mathbb{D}^{+}\left(\mathscr{P}^{+}\right)
$$

which is compatible with the natural isomorphism $\operatorname{Lie}(G) \cong \operatorname{Lie}\left(\mathscr{P}^{+}\right)$.

There is no analogue of Corollary 3.22 for $\Phi_{R}^{+}$because $\mathbb{D}^{+}\left(\mathscr{P}^{+}\right)$is only a crystal on $\operatorname{Cris}_{\mathrm{adm}}\left(R / \mathbb{Z}_{2}\right)$ and not on $\operatorname{Cris}_{\mathrm{adm}}(R)$, but see Corollary 4.10.

\section{From 2-divisible groups to Dieudonné displays}

In this section we construct a functor $\Phi_{R}$ from $p$-divisible groups over an admissible ring $R$ with $p=2$ to Dieudonné displays. When $2 R=0$, this has been done in the previous section, and the extension to all $R$ is unique, as will be shown in the end of this section. The construction relies on the following definition of divided powers on the ideal $\square_{R} \subseteq \mathbb{W}(R)$ when $4 R=0$.

4A. Divided powers on Zink rings. We note that for a $\mathbb{Z}_{(2)}$-algebra $B$ and an ideal $\mathfrak{b} \subseteq B$, divided powers on $\mathfrak{b}$ are equivalent to a map $\gamma: \mathfrak{b} \rightarrow \mathfrak{b}$ such that

$$
\begin{gathered}
\gamma(x y)=x^{2} \gamma(y) \text { for } x \in B \text { and } y \in \mathfrak{b}, \\
\gamma(x+y)=\gamma(x)+x y+\gamma(y) \text { for } x, y \in \mathfrak{b} .
\end{gathered}
$$

Here (4-1) and (4-2) also give $2 \gamma(x)=x^{2}$ for $x \in \mathfrak{b}$, since we can calculate

$$
4 \gamma(x)=\gamma(2 x)=\gamma(x+x)=2 \gamma(x)+x^{2} .
$$

For an admissible ring $R$ with $p=2$, the canonical divided powers on the ideal $I_{R} \subseteq W(R)$ defined by $\gamma(v(a))=v\left(a^{2}\right)$ induce divided powers on $\rrbracket_{R} \subseteq \mathbb{W}(R)$ only if $2 R=0$; see Section $1 \mathrm{G}$. Using $\vee$ instead of $v$ we get a little further.

Proposition 4.1. For an admissible ring $R$ with $p=2$ we consider the map

$$
\gamma: \rrbracket_{R} \rightarrow \rrbracket_{R}, \quad \gamma(\vee(a))=\vee\left(a^{2}\right) .
$$

If $4 R=0$, then $\gamma$ defines divided powers on $\square_{R}$ which are compatible with the canonical divided powers of 2 , and the corresponding extension of $\gamma$ to $\rrbracket_{R}+2 \mathbb{W}(R)$ is stable under the Frobenius $f$ of $\mathbb{W}(R)$.

If $8 R=0$, let $U \subseteq \mathbb{W}(R)$ be the set of all Witt vectors of the form $v([x])=$ $(0, x, 0, \ldots)$ with $x \in 4 R$. This is an ideal. Let $\widetilde{S}=\mathbb{W}(R) / U$. Then $\gamma$ induces divided powers on the ideal $\rrbracket_{R} / U$ of $\widetilde{S}$, which can naturally be extended to divided powers on $\square_{R} / U+2 \widetilde{S}$ that commute with the endomorphism $\sigma$ on $\widetilde{S}$ induced by $f$, and the extended divided powers stabilise the ideal $2 \widetilde{S}$. 
Proof. We will only consider the case $8 R=0$ and show that the extended divided powers satisfy $\gamma(2)=2-[4]$. Then the case $4 R=0$ follows.

Since $4 R$ is an ideal of square zero, we have $\widehat{W}(4 R)=(4 R)^{(\mathbb{N})}$ as $W(R)$ modules, where $W(R)$ acts on the $i$-th component of the right-hand side by the $i$-th Witt polynomial $w_{i}$, and $f$ annihilates $\widehat{W}(4 R)$. Thus $U$ is an ideal of $\mathbb{W}(R)$, and $f$ induces $\sigma: \widetilde{S} \rightarrow \widetilde{S}$. Let us show that $\gamma$ factors over a map $\rrbracket_{R} / U \rightarrow \rrbracket_{R}$. Indeed, for $a \in \mathbb{W}(R)$ and $x \in 4 R$ we have

$$
\gamma(\vee(a)+v([x]))=\gamma(\vee(a)+\vee([x]))=\vee\left((a+[x])^{2}\right)=\vee\left(a^{2}\right)=\gamma(\vee(a)) ;
$$

here $v([x])=\vee([x])$ because $u_{0}$ maps to 1 in $W\left(\mathbb{F}_{2}\right)$ and thus $u_{0}[x]=[x]$. Let us verify axiom (4-1) for the map $\gamma: \rrbracket_{R} \rightarrow \rrbracket_{R}$. For $a, b \in \mathbb{W}(R)$ we have

$$
\gamma(a \vee(b))=\gamma(\vee(f(a) b))=\vee\left(f\left(a^{2}\right) b^{2}\right)=a^{2} \vee\left(b^{2}\right)=a^{2} \gamma(\vee(b)) .
$$

Consider now axiom (4-2). For $a, b \in \mathbb{W}(R)$ we calculate

$$
\gamma(\vee(a)+\vee(b))=\vee\left((a+b)^{2}\right)=\gamma(\vee(a))+\vee(2 a b)+\gamma(\vee(b))
$$

so that $\vee(2 a b)$ has to be related with $\vee(a) \vee(b)$, which is

$$
\vee(a) \vee(b)=v\left(u_{0} a\right) v\left(u_{0} b\right)=v\left(2 u_{0}^{2} a b\right)=\vee\left(2 u_{0} a b\right) .
$$

Since $2 u_{0}=2-[4]$, we get

$$
\vee(2 a b)-\vee(a) \vee(b)=\vee([4] a b)=v\left(\left[4 a_{0} b_{0}\right]\right) \in U .
$$

Thus (4-2) holds for $\gamma: \rrbracket_{R} / U \rightarrow \rrbracket_{R} / U$, and $\gamma$ defines divided powers on this ideal. We want to extend these to divided powers on the ideal

$$
\tilde{I}=\rrbracket_{R} / U+2 \tilde{S}=\rrbracket_{R} / U+\widehat{W}(2 R) / U .
$$

Let

$$
\mathfrak{b}=\left\{\left(2 a_{0}, 4 a_{1}, 0, \ldots\right) \mid a_{i} \in R\right\} \subseteq \widehat{W}(2 R) .
$$

This is an ideal of $\mathbb{W}(R)$ with $\rrbracket_{R} \cap \mathfrak{b}=U$, and we have

$$
\tilde{I}=\rrbracket_{R} / U \oplus \mathfrak{b} / U \text {. }
$$

Thus the extension of $\gamma$ to $\widetilde{I}$ corresponds to giving arbitrary divided powers on $\mathfrak{b} / U \cong 2 R$. We take $\gamma([2 a])=\left[-2 a^{2}\right]$ for $a \in R$. Using $\vee(1)=2-[2]$ we obtain

$$
\gamma(2)=\gamma([2]+\vee(1))=[-2]+\vee(1)=[-2]+2-[2]=2-[4]
$$

in $\widetilde{S}$, as announced. Let us show that $\gamma \sigma=\sigma \gamma$ on $\widetilde{I}$ : for $a \in \mathbb{W}(R)$, we have

$$
\begin{aligned}
\gamma \sigma(\vee(a)) & =\gamma((2-[4]) a)=\gamma(2-[4]) a^{2}=\gamma(2) a^{2} \\
& =(2-[4]) a^{2}=\sigma\left(\vee\left(a^{2}\right)\right)=\sigma \gamma(\vee(a)) .
\end{aligned}
$$


Finally we have [4] $=2[2]$ in $\mathfrak{b} / U$, which implies that $\gamma(2) \in 2 \widetilde{S}$. This finishes the proof of Proposition 4.1.

Remark 4.2. The proof shows that the extension of $\gamma$ is uniquely determined by the condition that it commutes with $\sigma$. By choosing $\gamma([2 a])=\left[2 a^{2}\right]$, we get an extension with $\gamma(2)=2$ but which does not commute with $\sigma$.

Let $R$ be an admissible ring with $4 R=0$. Proposition 4.1 implies that its Dieudonné frame $\mathscr{D}_{R}$ satisfies the hypotheses of Construction 3.16 so that we obtain a functor

$\Phi_{R}^{o}:($ 2-divisible groups over $R) \rightarrow\left(\right.$ filtered $F-V$-modules over $\left.\mathscr{D}_{R}\right)$.

However, we cannot argue as in Theorem 3.19 in order to get a $\mathscr{D}_{R}$-window, because the divided powers on $\square_{R}$ do not exist for universal deformation rings, and thus Proposition 3.17 cannot be applied directly. The following modification will be sufficient for our purpose.

4B. A frame lift. Assume we are given a strict frame homomorphism

$$
\mathcal{F}^{\prime}=\left(S^{\prime}, I^{\prime}, R^{\prime}, \sigma^{\prime}, \sigma_{1}^{\prime}\right) \stackrel{\pi}{\longrightarrow} \mathcal{F}=\left(S, I, R, \sigma, \sigma_{1}\right)
$$

such that both $\pi: S^{\prime} \rightarrow S$ and $I^{\prime} \rightarrow I$ are surjective, and an ideal $U \subseteq \operatorname{Ker}(\pi)$ which is stable under $\sigma^{\prime}$. Let

$$
\widetilde{S}=S^{\prime} / U, \quad J=\operatorname{Ker}(\pi) / U, \quad \tilde{I}=\operatorname{Ker}(\widetilde{S} \rightarrow R) ;
$$

thus $S=\widetilde{S} / J$ and $R=\widetilde{S} / \tilde{I}$. Let $\tilde{\sigma}: \widetilde{S} \rightarrow \widetilde{S}$ be the homomorphism induced by $\sigma^{\prime}$, let $\theta^{\prime} \in S^{\prime}$ be the element defined by the relation $\sigma^{\prime}=\theta^{\prime} \sigma_{1}^{\prime}$ on $I^{\prime}$, and let $\tilde{\theta} \in \widetilde{S}$ and $\theta \in S$ be its images. We assume that $\mathcal{F}$ satisfies the conditions of Construction 3.16; i.e., $S$ and $R$ are $p$-adically complete, $I$ carries divided powers compatible with the canonical divided powers of $p$ and with $\sigma$, and $\theta=p$. Then Construction 3.16 gives a functor

$\Phi^{o}:(p$-divisible groups over $R) \rightarrow($ filtered $F-V$-modules over $\mathcal{F})$.

We also assume that the following conditions are satisfied.

(4-3) We have $\tilde{\sigma}(J)=0$ and $J=\{x \in \widetilde{S} \mid p x=0\}$.

(4-4) We have $\tilde{\theta}=p \tilde{u}$ for a unit $\tilde{u} \in \tilde{S}$.

(4-5) The ideal $\tilde{I}+p \widetilde{S}$ is equipped with divided powers which lift the given divided powers on $I+p S$, which commute with $\tilde{\sigma}$, and which stabilise the ideal $p \widetilde{S}$.

(4-6) There is an ideal $\mathfrak{a} \subseteq S$ with $\sigma(\mathfrak{a}) \subseteq \mathfrak{a} \subseteq \operatorname{Rad} S$ such that the $\operatorname{ring} S / \mathfrak{a}$ has no $p$-torsion. 
If $S$ has no $p$-torsion one can take $\mathcal{F}^{\prime}=\mathcal{F}$ and all axioms are clear. The following extends Proposition 3.17. Note that the prime $p$ is arbitrary here.

Proposition 4.3. In this situation there is a well-defined functor

$$
\Phi:(p \text {-divisible groups over } R) \rightarrow(\mathcal{F} \text {-windows })
$$

such that for $\Phi^{o}(G)=\left(P, Q, F^{\sharp}, V^{\sharp}\right)$ the filtered $F$ - $V$-module associated to $\Phi(G)$ is equal to $\left(P, Q, F^{\sharp}, u V^{\sharp}\right)$, where $u \in S$ is the image of $\tilde{u} \in \widetilde{S}$.

Proof. Conditions (4-3) and (4-4) imply that multiplication by $\tilde{\theta}$ on $\tilde{S}$ induces an injective map $\tilde{\theta}: S \rightarrow \widetilde{S}$ with image $\tilde{\theta} \widetilde{S}=p \widetilde{S}$. Moreover $\tilde{\sigma}$ induces a homomorphism $\tilde{\sigma}: S \rightarrow \tilde{S}$ that lifts $\sigma$. The relation $\theta^{\prime} \sigma_{1}^{\prime}=\sigma^{\prime}$ on $I^{\prime}$ gives $\tilde{\theta} \circ \sigma_{1}=\tilde{\sigma}$ as maps $I \rightarrow \tilde{S}$.

Let $G$ be a $p$-divisible group over $R$ and let $\Phi^{o}(G)$ be as above, i.e.,

$$
P=\mathbb{D}(G)_{S / R}=\mathbb{D}\left(G_{R_{0}}\right)_{S / R_{0}}
$$

with $R_{0}=R / p R$, the submodule $Q \subseteq P$ is the kernel of $P \rightarrow \operatorname{Lie} G$, and $F^{\sharp}$ and $V^{\sharp}$ are induced by the Verschiebung and Frobenius of $G_{R_{0}}$. The proof of [Kisin 2006, Lemma A.2] shows that $F(Q) \subseteq p P$. Let us recall the argument: for $S_{0}=S / p S$, the kernel of $S_{0} \rightarrow R_{0}$ is a nilideal because it carries divided powers. By [Illusie 1985, Théorème 4.4] there is a lift $G_{S_{0}}$ of $G_{R_{0}}$ to $S_{0}$, and we have $P=\mathbb{D}\left(G_{S_{0}}\right)_{S / S_{0}}$. Let $Q_{1}=\operatorname{Ker}\left(P \rightarrow\right.$ Lie $\left.G_{S_{0}}\right)$. Then $Q \subseteq Q_{1}+I P$, and the image of $F$ applied to both summands lies in $p P$.

Since $p J=0$ and $S$ is $p$-adically complete, so is $\widetilde{S}$. By (4-5) we can define

$$
\widetilde{P}=\mathbb{D}(G)_{\tilde{S} / R}=\mathbb{D}\left(G_{R_{0}}\right)_{\tilde{S} / R_{0}} .
$$

Here we use the (dual of the) Dieudonné crystal of [Mazur and Messing 1974, Chapter II §9], which is defined for divided power extensions that are not necessarily compatible with the canonical divided powers of $p$; see also [Berthelot et al. 1982, §1.4]. Let $\widetilde{Q} \subseteq \widetilde{P}$ be the kernel of $\widetilde{P} \rightarrow$ Lie $G$; this is the inverse image of $Q$ under the projection $\widetilde{P} \rightarrow \widetilde{P} / J \widetilde{P}=P$. Again, the Verschiebung and Frobenius of $G_{R_{0}}$ induce $\widetilde{S}$-linear maps $\widetilde{F}^{\sharp}: \widetilde{P}^{(\widetilde{\sigma})} \rightarrow \widetilde{P}$ and $\widetilde{V}^{\sharp}: \widetilde{P} \rightarrow \widetilde{P}^{(\widetilde{\sigma})}$. Since the divided powers stabilise the ideal $p \widetilde{S}$, the argument of [Kisin 2006, Lemma A.2] again shows that $\widetilde{F}(\widetilde{Q}) \subseteq p \widetilde{P}=\tilde{\theta} \widetilde{P}$, where $\widetilde{F}: \widetilde{P} \rightarrow \widetilde{P}$ is the $\tilde{\sigma}$-linear map corresponding to $\widetilde{F}^{\sharp}$. Since $\tilde{\sigma}$ annihilates $J$, the map $\widetilde{F}$ induces a map $\widetilde{F}: P \rightarrow \widetilde{P}$ which lifts $F$. Let $F_{1}: Q \rightarrow P$ be the composition

$$
F_{1}: Q \stackrel{\widetilde{F}}{\longrightarrow} \tilde{\theta} \widetilde{P} \stackrel{\tilde{\theta}}{\sim} P
$$

i.e., $F_{1}=\tilde{\theta}^{-1} \circ \tilde{F}$. We define $\Phi(G)=\left(P, Q, F, F_{1}\right)$. In order that this is an $\mathcal{F}$-window we have to verify that 
(i) for $x \in P$ and $a \in I$ we have $F_{1}(a x)=\sigma_{1}(a) F(x)$;

(ii) the image $F_{1}(Q)$ generates $P$.

Moreover, $u V^{\sharp}$ is the operator associated to $\Phi(G)$ as we have claimed if and only if (iii) for $x \in Q$ we have $u V^{\sharp}\left(F_{1}(x)\right)=1 \otimes x$ in $P^{(\sigma)}$.

The equation in (i) is equivalent to $\tilde{F}(a x)=\tilde{\theta}\left(\sigma_{1}(a) F(x)\right)$. Since $\tilde{F}(a x)=$ $\tilde{\sigma}(a) \tilde{F}(x)$ and $\tilde{\sigma}=\tilde{\theta} \circ \sigma_{1}$, this is clear. To prove (ii), it suffices to show that for each maximal ideal $\mathfrak{m}$ of $R$ and perfect field extension $R / \mathfrak{m} \subseteq k$ the vector space $\widetilde{P} \otimes \tilde{S} k$ is generated by $F_{1}(Q)$. Using (4-6) we get a sequence of $\sigma$-equivariant maps $S \rightarrow \widetilde{S} / \mathfrak{a} \rightarrow W(\widetilde{S} / \mathfrak{a}) \rightarrow W(k)$; the second arrow exists uniquely since $\widetilde{S} / \mathfrak{a}$ has no $p$-torsion and carries a Frobenius lift induced by $\tilde{\sigma}$; see [Bourbaki 1983, IX, §1.2, Proposition 2] and the explanation following [Zink 2001b, Theorem 4]. By functoriality we are reduced to the case where $\mathcal{F}^{\prime}=\mathcal{F}=\mathscr{W}_{k}$, which is classical. Assertion (iii) is equivalent to $\tilde{u} \tilde{V}^{\sharp}(\tilde{F}(x))=\tilde{\theta}(1 \otimes x)$ for $x \in Q$, which holds since $\widetilde{V}^{\sharp}(\tilde{F}(x))=p(1 \otimes x)$ in $\widetilde{P}^{(\tilde{\sigma})}$ for all $x \in \widetilde{P}$.

Now we construct an example for Proposition 4.3 with $p=2$. Let $A_{\text {red }}$ be a perfect ring of characteristic 2 and let $A=W\left(A_{\text {red }}\right) \llbracket t \rrbracket$, where $t$ is a finitely generated projective $W\left(A_{\text {red }}\right)$-module. Let $\mathfrak{m}=(2, t)$ be the kernel of $A \rightarrow A_{\text {red }}$. We write $A_{n}=A / 2^{n} A$ and $A_{n+}=A / 2^{n} \mathfrak{m}$. Only the rings

$$
A_{2+} \rightarrow A_{1+} \rightarrow A_{1}
$$

will play a role. We consider the frames $\mathcal{F}^{\prime}=\mathscr{D}_{A_{2+}} \rightarrow \mathcal{F}=\mathscr{D}_{A_{1+}}$, i.e.,

$$
\begin{array}{rlrl}
S & =\mathbb{W}\left(A_{1+}\right), \quad I=\rrbracket_{A_{1+}}, & R & =A_{1+}, \\
S^{\prime} & =\mathbb{W}\left(A_{2+}\right), \quad I^{\prime}=\rrbracket_{A_{2+}}, & R^{\prime}=A_{2+} .
\end{array}
$$

Then $\theta^{\prime}=2-$ [4] in $S^{\prime}$ and thus $\theta=2$ in $S$. Let $U \subseteq S^{\prime}$ be the ideal of all Witt vectors $v([x])$ with $x \in 4 A_{2+}$, and let $\widetilde{S}=S^{\prime} / U$. As above, we write

$$
J=\operatorname{Ker}(\widetilde{S} \rightarrow S)=\widehat{W}(2 \mathfrak{m} / 4 \mathfrak{m}) / U
$$

and

$$
\widetilde{I}=\operatorname{Ker}(\widetilde{S} \rightarrow R)=\left(I^{\prime}+\widehat{W}(2 \mathfrak{m} / 4 \mathfrak{m})\right) / U .
$$

Proposition 4.4. These data satisfy the axioms (4-3)-(4-6).

Proof. The divided powers required in (4-5) are given by Proposition 4.1. Since $2 \mathfrak{m} / 4 \mathfrak{m} \subseteq A_{2+}$ is an ideal of square zero, we have

$$
J^{\prime}:=\widehat{W}(2 \mathfrak{m} / 4 \mathfrak{m})=(2 \mathfrak{m} / 4 \mathfrak{m})^{(\mathbb{N})}
$$

as $W\left(A_{2+}\right)$-modules, where $W\left(A_{2+}\right)$ acts on the $i$-th component of the right-hand side via the $i$-th Witt polynomial. We have $\sigma^{\prime}\left(J^{\prime}\right)=2 J^{\prime}=0$ and $J=J^{\prime} / U$. Thus $\tilde{\sigma}: \widetilde{S} \rightarrow \widetilde{S}$ is defined and vanishes on $J$, and $2 J=0$. 
Lemma 4.5. Multiplication by 2 induces an isomorphism of groups

$$
\widehat{W}\left(2 A_{1+}\right) \stackrel{\sim}{\longrightarrow}\left(4 A_{2+}\right) / U .
$$

Proof. The divided Witt polynomials for the canonical divided powers of 2 give an isomorphism $\log : W(2 A) \cong 2 A^{\mathbb{N}}$. The composition

$$
W(2 A) \stackrel{\log }{\longrightarrow} 2 A^{\mathbb{N}} \longrightarrow(2 A / 4 A)^{\mathbb{N}}
$$

is given by $\left(2 a_{0}, 2 a_{1}, \ldots\right) \mapsto 2\left[a_{0}, a_{0}^{2}+a_{1}, a_{1}^{2}+a_{2}, \ldots\right]$, while the composition

$$
W(4 A) \stackrel{\log }{\longrightarrow} 4 A^{\mathbb{N}} \longrightarrow(4 A / 8 A)^{\mathbb{N}}
$$

is simply $\left(4 a_{0}, 4 a_{1}, \ldots\right) \mapsto 4\left[a_{0}, a_{1}, \ldots\right]$. It follows that the homomorphism of the lemma is isomorphic to the homomorphism

$$
A_{\text {red }}^{(\mathbb{N})} \rightarrow A_{\text {red }}^{(\mathbb{N})}, \quad\left(a_{0}, a_{1}, \ldots\right) \mapsto\left(a_{0}, a_{1}^{2}+a_{2}, a_{2}^{2}+a_{3}, \ldots\right) .
$$

Since $A_{\text {red }}$ is perfect this map is bijective.

Let us continue with the proof of Proposition 4.4. To verify (4-3), let $x \in \widetilde{S}$ with $2 x=0$. Since $\mathbb{W}\left(A_{1}\right)$ has no 2-torsion we have $x \in \widehat{W}\left(2 A_{2+}\right) / U$. Lemma 4.5 implies that $x \in J$, and (4-3) is proved. Let $u=1-$ [2] in $\mathbb{W}\left(A_{2+}\right)$, which is a unit. By the proof of Lemma 4.5 we have $2 u=2-(4,4,0, \ldots) \equiv 2-[4]=\theta^{\prime}$ modulo $U$, which proves (4-4). In (4-6) we can take $\mathfrak{a}=\widehat{W}\left(2 A_{1+}\right)$.

4C. The Dieudonné display associated to a 2-divisible group. Let $p=2$ and let $u=1-[2]$ in $\mathbb{W}\left(\mathbb{Z}_{2}\right)$. We begin to construct the functor $\Phi_{R}$ in an initial case. Recall that $\Phi_{R}^{o}$ was defined in the end of Section 4 A when $4 R=0$.

Proposition 4.6. For each admissible ring $R$ with $p=2$ and $2 \mathcal{N}_{R}=0$ there is a functor

$$
\Phi_{R}:(\text { 2-divisible groups over } R) \rightarrow\left(\mathscr{D}_{R} \text {-windows }\right)
$$

compatible with base change in $R$ such that for $\Phi_{R}^{o}(G)=\left(P, Q, F^{\sharp}, V^{\sharp}\right)$ the filtered $F$-V-module associated to $\Phi_{R}(G)$ is equal to $\left(P, Q, F^{\sharp}, u V^{\sharp}\right)$.

Proof. This is similar to the proof of Theorem 3.19. Propositions 4.3 and 4.4 give the desired system of functors $\Phi_{R}$ for topological admissible rings $R$ of the type $R=A_{1+}$ as above. For a $p$-divisible group $G$ over an admissible ring $R$ as in the proposition, let $\Lambda=W\left(R_{\text {red }}\right)$ and $\bar{G}=G \otimes_{R} R_{\text {red }}$. Let $A$ be the $\Lambda$-algebra that prorepresents the functor $\operatorname{Def}_{\bar{G}}$ on $\mathrm{Nil}_{\Lambda / K}$ (this $A$ was denoted by $B$ in Section 3), let $\mathscr{G}$ over $A$ be the universal deformation, and let $\mathscr{G}_{1+}=\mathscr{G} \otimes_{A} A_{1+}$. The unique homomorphism of $\Lambda$-algebras $A \rightarrow R$ with $G=\mathscr{G} \otimes_{A} R$ as deformations of $\bar{G}$ factors over a homomorphism $A_{1+} \rightarrow R$, and we define $\Phi_{R}(G)$ as the base change of $\Phi_{A_{1+}}\left(\mathscr{G}_{1+}\right)$ under this map. We have to show that the operator $F_{1}$ attached to 
$\Phi_{R}^{o}(G)$ in this way is functorial in $G$ and in $R$. This is analogous to the proof of Theorem 3.19, using that $F_{1}$ is functorial with respect to homomorphisms of rings of the type $A_{1+}$.

For an admissible ring $R$, let $i: \mathscr{D}_{R} \rightarrow \mathscr{D}_{R}^{+}$be the natural homomorphism.

Proposition 4.7. Let $R$ be an admissible ring with $p=2$ and $2 \mathcal{N}_{R}=0$. For each 2-divisible group $G$ over $R$ there is a natural isomorphism of $\mathscr{D}_{R}^{+}$-windows

$$
\Phi_{R}^{+}(G) \cong i_{*} \Phi_{R}(G)
$$

The functor $\Phi_{R}^{+}$was defined in Proposition 3.23.

Proof. Let us write $\Psi_{R}(G)=i_{*} \Phi_{R}(G)$ so that we have two functors

$$
\left.\Phi_{R}^{+}, \Psi_{R}: \text { (2-divisible groups over } R\right) \rightarrow\left(\mathscr{D}_{R}^{+}\right. \text {-windows). }
$$

When $2 R=0$, thus $\mathscr{D}_{R}=\mathscr{D}_{R}^{+}$, these functors coincide by the uniqueness assertion of Corollary 3.24. The rest is quite formal. Let $R_{1}=R / 2 R$. For a $p$-divisible group $G$ over $R$, let $G_{1}=G \otimes_{R} R_{1}$ and $\bar{G}=G \otimes_{R} R_{\text {red }}$. The canonical divided powers of 2 make $R \rightarrow R_{1}$ into a divided power extension. By Corollaries 2.11 and 3.26, the $\mathscr{D}_{R}^{+}$-windows $\Phi_{R}^{+}(G)$ and $\Psi_{R}(G)$ correspond to two lifts of the Hodge filtration of $G$ to $\mathbb{D}\left(G_{1}\right)_{R / R_{1}}$. Their difference is measured by a homomorphism of $R_{1}$-modules

$$
h_{G}^{\prime}: V\left(G_{1}\right) \rightarrow \operatorname{Lie}\left(G_{1}\right) \otimes_{R} 2 R,
$$

where $V(G)$ is the kernel of $\mathbb{D}(G)_{R} \rightarrow \operatorname{Lie}(G)$. We have to show that $h_{G}^{\prime}$ is zero for all $G$. Since $h_{G}^{\prime}$ is functorial in $R$ we may assume that $R=A_{1+} / \mathfrak{m}^{n}$ for some $n \geq 2$, where $A$ is the universal deformation ring of $\bar{G}$. Then $2 R$ is a free

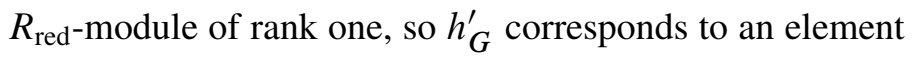

$$
h_{G} \in \operatorname{Hom}(V(\bar{G}), \operatorname{Lie}(\bar{G})) .
$$

Now an injective homomorphism $R_{\text {red }} \rightarrow R_{\text {red }}^{\prime}$ gives an injective homomorphism of the associated rings $R \rightarrow R^{\prime}$, while a product decomposition $R_{\text {red }}=\prod R_{i \text {,red }}$ gives $R=\prod R_{i}$. Since $R_{\text {red }}$ embeds into the product of its localisations at minimal prime ideals, we may assume that $k:=R_{\text {red }}$ is a field. There is a deformation $\bar{G}^{\prime}$ of $\bar{G}$ over $R_{\text {red }}^{\prime}:=k \llbracket x \rrbracket^{\text {per }}$ with ordinary generic fibre. Let $A^{\prime}$ be its universal deformation ring and let $G^{\prime}$ over $R^{\prime}=A_{1+}^{\prime} / \mathfrak{m}^{n}$ be given by the universal deformation. By functoriality it suffices to show that $h_{G^{\prime}}=0$. Again we can pass to the field of fractions $k((x))^{\mathrm{per}}$. Thus we are left to show that $h_{G}=0$ if $G$ is ordinary over $R=A_{1+} / \mathfrak{m}^{n}$, where $k=R_{\text {red }}$ is a perfect field. There is a deformation $G^{\prime \prime}$ of $G_{k}$ over $R^{\prime \prime}:=W_{2}(k)$ which decomposes into the direct sum of its étale and multiplicative part. Let $R \rightarrow R^{\prime \prime}$ be the unique homomorphism such that $G^{\prime \prime}=G \otimes_{R} R^{\prime \prime}$ as deformations of $G_{k}$. Since this does not change $h_{G}$ we may 
replace $G$ by $G^{\prime \prime}$. Since $\Psi_{R}$ and $\Phi_{R}^{+}$both preserve direct sums we may assume that $G$ is étale or of multiplicative type. Then $h_{G}$ vanishes since $\operatorname{Hom}(V(\bar{G}), \operatorname{Lie}(\bar{G}))$ is zero.

Lemma 4.8. Let $R$ be an admissible ring with $p=2$ and let $R_{1+}=R / 2 \mathcal{N}_{R}$. The commutative diagram of frames

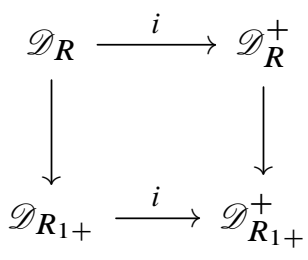

is Cartesian on each component of the frames, and the associated diagram of window categories is 2-Cartesian.

Proof. The vertical arrows are surjective, and the horizontal arrows are injective with equal cokernel by Lemma 1.10 and its proof. Thus the diagram of frames is Cartesian on each component. For a ring $A$, let $\mathrm{V}(A)$ be the category of projective $A$-modules of finite type. The functor

$$
\mathrm{V}(\mathbb{W}(R)) \rightarrow \mathrm{V}\left(\mathbb{W}^{+}(R)\right) \times_{\mathrm{V}\left(W^{+}\left(R_{1+}\right)\right)} \mathrm{V}\left(\mathbb{W}\left(R_{1+}\right)\right)
$$

is fully faithful since the diagram is Cartesian, and it is essentially surjective since $\mathrm{V}(\mathbb{W}(R)) \rightarrow \mathrm{V}\left(\mathbb{W}\left(R_{1+}\right)\right)$ and $\mathrm{V}\left(\mathbb{W}^{+}(R)\right) \rightarrow \mathrm{V}\left(\mathbb{W}^{+}\left(R_{1+}\right)\right)$ are bijective on isomorphism classes and surjective on automorphism groups. It follows easily that the diagram of window categories is 2-Cartesian.

Theorem 4.9. For each admissible ring $R$ with $p=2$ there is a functor

$$
\Phi_{R}:(\text { 2-divisible groups over } R) \rightarrow\left(\mathscr{D}_{R} \text {-windows }\right)
$$

compatible with base change in $R$ such that $\Phi_{R}$ is given by Proposition 4.6 when $2 \mathcal{N}_{R}=0$, and such that there is a natural isomorphism of $\mathscr{D}_{R}^{+}$-windows

$$
\Phi_{R}^{+}(G) \cong i_{*} \Phi_{R}(G)
$$

Proof. This is clear from Propositions 3.23 and 4.7 and Lemma 4.8.

Corollary 4.10. Let $p=2$. For each 2-divisible group $G$ over an admissible ring $R$ with associated Dieudonné display $\mathscr{P}=\Phi_{R}(G)$, there is a natural isomorphism of crystals on Cris $_{\text {adm }}(R)$

$$
\mathbb{D}(G) \cong \mathbb{D}(\mathscr{P})
$$

which is compatible with the natural isomorphism $\operatorname{Lie}(G) \cong \operatorname{Lie}(\mathscr{P})$. 
Proof. We have a natural isomorphism of crystals on $\operatorname{Cris}_{\mathrm{adm}}\left(R / \mathbb{Z}_{2}\right)$

$$
\mathbb{D}(G) \cong \mathbb{D}\left(\Phi_{R}^{+}(G)\right) \cong \mathbb{D}\left(\Phi_{R}(G)\right)
$$

by Corollary 3.26, Theorem 4.9, and Lemma 2.12. The isomorphism of crystals on Cris $_{\text {adm }}(R)$ follows as in the proof of Corollary 3.22.

\section{D. Uniqueness of the functor $\Phi_{R}$.}

Proposition 4.11. Assume that for each admissible ring $R$ with $p=2$ we have a functor

$$
\Phi_{R}^{\prime}:(2 \text {-divisible groups over } R) \rightarrow\left(\mathscr{D}_{R} \text {-windows }\right)
$$

compatible with base change in $R$ such that $\Phi_{R}^{\prime}=\Phi_{R}$ when $2 R=0$. Then there is a natural isomorphism $\Phi_{R}^{\prime} \cong \Phi_{R}$ which is functorial in $R$ and equal to the identity when $2 R=0$.

Proof. We first show that $\Phi_{R}^{\prime} \cong \Phi_{R}$ when $4 R=0$. Let $R_{1}=R / 2 R$. For a $p$-divisible group $G$ over $R$, let $G_{1}=G \otimes_{R} R_{1}$ and let

$$
\mathscr{P}_{1}=\Phi_{R_{1}}\left(G_{1}\right)=\left(P, Q, F, F_{1}\right)
$$

be its Dieudonné display. If we take the trivial divided powers on the ideal $2 R$, Corollary 2.10 implies that the difference between $\Phi_{R}(G)$ and $\Phi_{R}^{\prime}(G)$ as lifts of $\mathscr{P}_{1}$ is measured by a homomorphism

$$
h_{G}^{\prime}: Q / \rrbracket_{R_{1}} P \rightarrow P / Q \otimes_{R_{1}} 2 R .
$$

Let $V(G)=\operatorname{Lie}\left(G^{\vee}\right)^{\vee}$. By Corollary 3.24 and by the construction of $\Phi_{R}^{o}(G)$ we can view $h_{G}^{\prime}$ as a homomorphism

$$
h_{G}: V\left(G_{1}\right) \rightarrow \operatorname{Lie}\left(G_{1}\right) \otimes_{R_{1}} 2 R .
$$

We want to show that $h_{G}=0$. We may assume that $R=A /\left(\mathfrak{m}^{n}+4 A\right)$, where $A$ is the universal deformation ring of $G \otimes_{R} R_{\text {red }}$ and $\mathfrak{m}$ is the kernel of $A \rightarrow R_{\text {red. }}$. As in the proof of Proposition 4.7, one reduces to the case where $k=R_{\text {red }}$ is a field and $G$ is ordinary. Assume that $G$ is an extension $0 \longrightarrow \mu_{p} \infty \longrightarrow G \longrightarrow \mathbb{Q}_{p} / \mathbb{Z}_{p} \longrightarrow 0$. Then $V(G)=V\left(\mathbb{Q}_{p} / \mathbb{Z}_{p}\right)=R$ and $\operatorname{Lie}(G)=\operatorname{Lie}\left(\mu_{p} \infty\right)=R$, so that $h_{G} \in 2 R$. Thus $G \mapsto h_{G}$ defines a map $g: \underline{\operatorname{Ext}}^{1}\left(\mathbb{Q}_{p} / \mathbb{Z}_{p}, \mu_{p} \infty\right) \rightarrow \mathbb{G}_{a}$ of functors on the category of local Artin rings with residue field $k$ and annihilated by 4 . It is easy to see that $g$ is additive. Here $\underline{\operatorname{Ext}}^{1}()=\mu_{p} \infty$, and it follows that $g=0$. This implies easily that $h_{G}^{\prime}=0$ when $G$ is ordinary. Thus $\Phi_{R} \cong \Phi_{R}^{\prime}$ when $4 R=0$. If for some $n \geq 1$ we know that $\Phi_{R} \cong \Phi_{R}^{\prime}$ when $2^{n} R=0$, the same reasoning shows that $\Phi_{R} \cong \Phi_{R}^{\prime}$ when $2^{n+1} R=0$, and the proposition follows. 


\section{Equivalence of categories}

Let $R$ be an admissible ring. Dieudonné displays over $R_{\text {red }}$ are displays, and they are equivalent to Dieudonné modules over $R_{\text {red }}$ by Lemma 2.4. Under this equivalence, the functor $\Phi_{R_{\text {red }}}$ corresponds to $\Phi_{R_{\text {red }}}^{o}$.

Proposition 5.1. For an admissible ring $R$ the following diagram of categories is 2-Cartesian:

( $p$-divisible groups over $R) \stackrel{\Phi_{R}}{\longrightarrow}$ (Dieudonné displays over $\left.R\right)$

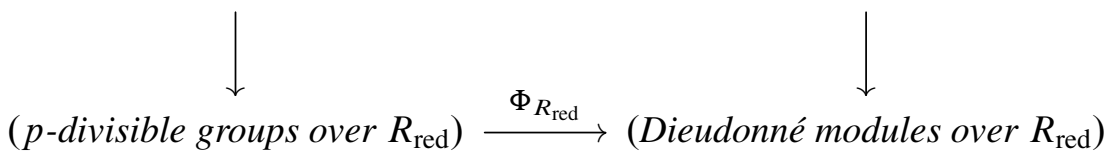

Proof. The categories of $p$-divisible groups and Dieudonné displays over $R$ are the direct limit of the corresponding categories over all finitely generated $W\left(R_{\text {red }}\right)$ algebras contained in $R$; see Section 3A. Thus we may assume that the ideal $\mathcal{N}_{R}$ is nilpotent. If $\mathfrak{a} \subseteq R$ is an ideal equipped with nilpotent divided powers and if the proposition holds for $R / \mathfrak{a}$, then it holds for $R$. This follows from the comparison of crystals in Corollaries 3.21 and 4.10, since lifts from $R / \mathfrak{a}$ to $R$ of $p$-divisible groups and of Dieudonné displays are both classified by lifts of the Hodge filtration by [Messing 1972] and by Corollary 2.10. When $\mathfrak{a}^{2}=0$, we can take the trivial divided powers on $\mathfrak{a}$. The result follows by induction on the order of nilpotence of $\mathcal{N}_{R}$.

Remark 5.2. Since $p$-divisible groups and Dieudonné displays over a perfect ring $K$ have universal deformation rings which are twisted power series rings over $\Lambda=$ $W(K)$, in order to prove Proposition 5.1 the case $R=K[\varepsilon]$ is sufficient. In particular, for $p=2$ this means that as soon as the functors $\Phi_{R}$ defined in Corollary 3.24 when $2 R=0$ are known to exist for all $R$, Proposition 5.1 is automatic. This reasoning does not apply to the functors $\Phi_{R}^{+}$(which also extend the functors $\Phi_{R}$ for $2 R=0$ to all $R$ but which are not an equivalence in general) because the deformation functors of $v$-stabilised Dieudonné displays are not prorepresentable.

We have the following result of Gabber, which is classical when $R_{\text {red }}$ is a field. It is also proved in [Lau 2013, Corollary 6.5].

Theorem 5.3. The functor $\Phi_{R_{\mathrm{red}}}$ is an equivalence of categories.

Corollary 5.4. For every admissible ring $R$ the functor $\Phi_{R}$ is an equivalence of exact categories.

Proof. By Theorem 5.3 and Proposition 5.1, the functor $\Phi_{R}$ is an equivalence of categories. A short sequence $0 \longrightarrow A \longrightarrow B \longrightarrow C \longrightarrow 0$ of $p$-divisible groups or of Dieudonné displays over $R$ is exact if and only if all its scalar extensions to 
perfect fields are exact. Thus $\Phi_{R}$ and its inverse preserve exact sequences, since this holds over perfect fields.

This proves Theorem A. Using Lemmas 2.15 and 2.16, we also get:

Corollary 5.5. For every admissible topological ring $R$ with a countable base of topology, p-divisible groups over $R$ are equivalent to Dieudonné displays over $R$.

Finally we note the following consequence of the crystalline duality theorem. The duality of windows is recalled in the end of Section 2A.

Corollary 5.6. Let $G$ be a p-divisible group over an admissible ring $R$ and let $G^{\vee}$ be its Cartier dual. There is a natural isomorphism

$$
\Phi_{R}\left(G^{\vee}\right) \cong \Phi_{R}(G)^{t} .
$$

Proof. Assume first that $p$ is odd. The crystalline duality theorem [Berthelot et al. $1982,5.3]$ gives an isomorphism of filtered $F$ - $V$-modules $\Phi_{R}^{o}\left(G^{\vee}\right)^{t} \cong \Phi_{R}^{o}(G)$. Since the functor from windows to filtered $F-V$-modules preserves duality, the uniqueness part of Theorem 3.19 implies that this isomorphism preserves $F_{1}$, i.e., it is an isomorphism of Dieudonné displays $\Phi_{R}\left(G^{\vee}\right)^{t} \cong \Phi_{R}(G)$. For $p=2$, using the uniqueness part of Corollary 3.24 we similarly get an isomorphism of Dieudonné displays $\Phi_{R}\left(G^{\vee}\right)^{t} \cong \Phi_{R}(G)$ when $2 R=0$. Then Proposition 4.11 gives such an isomorphism for all $R$.

\section{Breuil-Kisin modules}

We recall the main construction of [Lau 2010] without restriction on $p$. Let $R$ be a complete regular local ring with maximal ideal $\mathfrak{m}_{R}$ and with perfect residue field $k$ of characteristic $p$. Choose a representation $R=\mathfrak{S} / E \mathfrak{S}$ with

$$
\mathfrak{S}=W(k) \llbracket x_{1}, \ldots, x_{r} \rrbracket
$$

such that $E$ is a power series with constant term $p$. Let $J \subset \mathfrak{S}$ be the ideal generated by $x_{1}, \ldots, x_{r}$. Choose a ring endomorphism $\sigma: \mathfrak{S} \rightarrow \mathfrak{S}$ which lifts the Frobenius of $\mathfrak{S} / p \mathfrak{S}$ such that $\sigma(J) \subseteq J$. Let $\sigma_{1}: E \mathfrak{S} \rightarrow \mathfrak{S}$ be defined by $\sigma_{1}(E x)=\sigma(x)$ for $x \in \mathfrak{S}$. These data define a frame

$$
\mathscr{B}=\left(\mathfrak{S}, E \mathfrak{S}, R, \sigma, \sigma_{1}\right) .
$$

For each positive integer $a$, let $R_{a}=R / \mathfrak{m}_{R}^{a}$ and $\mathfrak{S}_{a}=\mathfrak{S} / J^{a}$. We have frames

$$
\mathscr{B}_{a}=\left(\mathfrak{S}_{a}, E \mathfrak{S}_{a}, R_{a}, \sigma, \sigma_{1}\right),
$$

where $\sigma$ and $\sigma_{1}$ are induced by the corresponding operators of $\mathscr{B}$.

The frames $\mathscr{B}$ and $\mathscr{B} a$ are related with the Witt and Dieudonné frames of $R$ and of $R_{a}$ as follows. Let $\delta: \mathfrak{S} \rightarrow W(\mathfrak{S})$ be the unique lift of the identity of $\mathfrak{S}$ 
such that $f \delta=\delta \sigma$, or equivalently $w_{n} \delta=\sigma^{n}$ for $n \geq 0$; see [Bourbaki 1983, IX, $\S 1.2$, Proposition 2] and the explanation following [Zink 2001b, Theorem 4]. The composition of $\delta$ with the projection $W(\mathfrak{S}) \rightarrow W(R)$ is a ring homomorphism

$$
\varkappa: \mathfrak{S} \rightarrow W(R)
$$

which lifts the projection $\mathfrak{S} \rightarrow R$ such that $f \varkappa=\varkappa \sigma$. The same construction gives compatible homomorphisms

$$
\varkappa_{a}: \mathfrak{S}_{a} \rightarrow W\left(R_{a}\right)
$$

for $a \geq 1$, which induce $\varkappa$ in the projective limit. Since the element $\varkappa(E)$ maps to zero in $R$ it lies in the image of $v: W(R) \rightarrow W(R)$. Let

$$
u=v^{-1}(\varkappa(E))=f_{1}(\varkappa(E)) .
$$

We will denote the image of $u$ in $W\left(R_{a}\right)$ also by $u$.

Lemma 6.1. The element $u \in W(R)$ is a unit. The homomorphisms $\varkappa$ and $\varkappa_{a}$ are $u$-homomorphisms of frames $\varkappa: \mathscr{B} \rightarrow \mathscr{W}_{R}$ and $\varkappa_{a}: \mathscr{B}_{a} \rightarrow \mathscr{W}_{R_{a}}$.

Proof. See [Lau 2010, Proposition 6.1]. Since $W(R) \rightarrow W(k)$ is a local homomorphism, in order to show that $u$ is a unit we can work with $\varkappa_{1}$, i.e., consider the case where $R=k$ and $\mathfrak{S}=W(k)$. Then $E=p$ and $u=1$. In order that $\varkappa$ and $\varkappa_{a}$ are $u$-homomorphisms of frames we need that $f_{1} \varkappa=u \cdot \varkappa \sigma_{1}$. For $x \in \mathfrak{S}$ we calculate $f_{1}(\varkappa(E x))=f_{1}(\varkappa(E) \varkappa(x))=f_{1}(\varkappa(E)) \cdot f(\varkappa(x))=u \cdot \varkappa(\sigma(x))=u \cdot \varkappa\left(\sigma_{1}(E x)\right)$, as required.

Let $\bar{\sigma}$ be the semilinear endomorphism of the free $W(k)$-module $J / J^{2}$ induced by $\sigma$. Since $\sigma$ induces the Frobenius modulo $p, \bar{\sigma}$ is divisible by $p$.

Proposition 6.2. The following conditions are equivalent:

(i) The image of $x: \mathfrak{S} \rightarrow W(R)$ lies in $\mathbb{W}(R)$.

(ii) The image of $\delta: \mathfrak{S} \rightarrow W(\mathfrak{S})$ lies in $\mathbb{W}(\mathfrak{S})$.

(iii) The endomorphism $p^{-1} \bar{\sigma}$ of $J / J^{2}$ is nilpotent modulo $p$.

Remark 6.3. In the special case $\sigma\left(x_{i}\right)=x_{i}^{p}$ the conditions of Proposition 6.2 hold. This is easy to see directly: we have $\delta\left(x_{i}\right)=\left[x_{i}\right]$, which gives (i) and (ii), moreover (iii) holds since $\bar{\sigma}$ is zero.

Proof of Proposition 6.2. For odd $p$ the equivalence between (i) and (iii) is [Lau 2010, Proposition 9.1]; its proof shows that (i) $\Longrightarrow$ (iii) $\Longrightarrow$ (ii) $\Longrightarrow$ (i). The proof also applies for $p=2$ if [Lau 2010, Lemma 9.2] is replaced by Lemma 6.4. 
Lemma 6.4. For $x \in \mathfrak{S}$ let $\tau(x)=\left(\sigma(x)-x^{p}\right) / p$. Let $\mathfrak{m}$ be the maximal ideal of $\mathfrak{S}$. For $n \geq 0$, the map $\tau$ preserves $\mathfrak{m}^{n} J$ and induces a $\sigma$-linear endomorphism $\operatorname{gr}_{n}(\tau)$ of the $k$-module $\operatorname{gr}_{n}(J)=\mathfrak{m}^{n} J / \mathfrak{m}^{n+1} J$. The endomorphism $\operatorname{gr}_{0}(\tau)$ is equal to $p^{-1} \bar{\sigma}$ modulo $p$. For $n \geq 1$, there is a surjective $k$-linear map

$$
\pi_{n}: \operatorname{gr}_{n}(J) \rightarrow \operatorname{gr}_{0}(J)
$$

such that $\operatorname{gr}_{0}(\tau) \pi_{n}=\pi_{n} \operatorname{gr}_{n}(\tau)$ and such that $\operatorname{gr}_{n}(\tau)$ vanishes on $\operatorname{Ker}\left(\pi_{n}\right)$. In particular, $p^{-1} \bar{\sigma}$ is nilpotent modulo $p$ if and only if $\operatorname{gr}_{0}(\tau)$ is nilpotent, which implies that $\operatorname{gr}_{n}(\tau)$ is nilpotent for each $n$.

Proof. We have $\sigma(J) \subseteq J^{p}+p J \subseteq \mathfrak{m} J$ and thus $\sigma\left(\mathfrak{m}^{n} J\right) \subseteq \mathfrak{m}^{n+1} J$. It follows that $p \tau\left(\mathfrak{m}^{n} J\right) \subseteq p \mathfrak{S} \cap \mathfrak{m}^{n+1} J=p \mathfrak{m}^{n} J$ and $\tau\left(\mathfrak{m}^{n} J\right) \subseteq \mathfrak{m}^{n} J$. For $x, y \in \mathfrak{m}^{n} J$ the element $\tau(x+y)-\tau(x)-\tau(y)$ is a multiple of $x y$ and thus lies in $\mathfrak{m}^{2 n+1} J$. Hence $\tau$ induces an additive endomorphism $\operatorname{gr}_{n}(\tau)$ of $\operatorname{gr}_{n}(J)$. It is $\sigma$-linear because for $a \in \mathfrak{S}$ and $x \in \mathfrak{m}^{n} J$ the element $\tau(a x)-\sigma(a) \tau(x)=\tau(a) x^{p}$ lies in $\mathfrak{m}^{p n} J^{p} \subseteq \mathfrak{m}^{n+1} J$. Let us write $\sigma\left(x_{i}\right)=x_{i}^{p}+p y_{i}$ with $y_{i} \in J$. We have $\tau\left(x_{i}\right)=y_{i}$ and $p^{-1} \bar{\sigma}\left(x_{i}\right) \equiv y_{i}$ modulo $J^{2}$. Thus $\operatorname{gr}_{0}(\tau)$ coincides with $p^{-1} \bar{\sigma}$ modulo $p$.

For each $n \geq 0$, a basis of $\operatorname{gr}_{n}(J)$ is given by all elements $p^{b} \underline{x} \underline{\underline{c}}$ with $\underline{c} \in \mathbb{N}^{r}$ and $1 \leq|\underline{c}| \leq n+1$ and $b+|\underline{c}|=n+1$. Let $n \geq 1$, and define $\pi_{n}$ to be the $k$-linear map with $\pi_{n}\left(p^{n} x_{i}\right)=x_{i}$ and $\pi_{n}\left(p^{b} \underline{\underline{c}} \underline{\underline{c}}\right)=0$ if $|\underline{c}|>1$. Then $\operatorname{gr}_{n}(\tau)$ vanishes on $\operatorname{Ker}\left(\pi_{n}\right)$ because $\sigma(J) \subseteq \mathfrak{m} J$, thus $\sigma\left(J^{2}\right) \subseteq \mathfrak{m}^{2} J^{2}$, and because for $x \in \mathfrak{m}^{n} J$ we have $x^{p} \in$ $\mathfrak{m}^{n+2} J$. The relation $\operatorname{gr}_{0}(\tau) \pi_{n}=\pi_{n} \operatorname{gr}_{n}(\tau)$ holds since $\tau\left(p^{n} x_{i}\right) \equiv p^{n-1} x_{i}^{p}+p^{n} y_{i}$ modulo $\mathfrak{m}^{n+1}(J)$. The last assertion of the lemma is immediate.

Lemma 6.5. If the equivalent conditions of Proposition 6.2 hold, then $\varkappa_{\text {and }} \varkappa_{a}$ are u-homomorphisms of frames

$$
\varkappa: \mathscr{B} \rightarrow \mathscr{D}_{R}, \quad \varkappa_{a}: \mathscr{B}_{a} \rightarrow \mathscr{D}_{R_{a}},
$$

where the unit $u \in \mathbb{W}(R)$ is given by

$$
u=v^{-1}(\varkappa(E))=\mathbb{F}_{1}(\varkappa(E)) .
$$

In $W(R)$ we have $u=u$ if $p$ is odd and $u=\left(v^{-1}(2-[2])\right)^{-1} u$ if $p=2$.

Proof. The proof of Lemma 6.1 with $f_{1}$ replaced by $\mathbb{I}_{1}$ shows that $u$ is a unit of $\mathbb{W}(R)$ and that $x$ and $\varkappa_{a}$ are $u$-homomorphisms of frames as indicated. The relation between $u$ and $u$ follows from the fact that $\mathscr{D}_{R} \rightarrow \mathscr{W}_{R}$ is a $u_{0}$-homomorphism, where $u_{0}=1$ if $p$ is odd and $v\left(u_{0}\right)=2-$ [2] if $p=2$.

Theorem 6.6. If the equivalent conditions of Proposition 6.2 hold, the frame homomorphisms $\varkappa: \mathscr{B} \rightarrow \mathscr{D}_{R}$ and $\varkappa_{a}: \mathscr{B}_{a} \rightarrow \mathscr{D}_{R_{a}}$ are crystalline.

Proof. The proof for odd $p$ in [Lau 2010, Theorem 9.3] works almost literally for $p=2$ as well. Let us recall the essential parts of the argument. Fix an integer 
$a \geq 1$. One can define a factorisation of the projection $\mathscr{B}_{a+1} \rightarrow \mathscr{B}_{a}$ into strict frame homomorphisms

$$
\mathscr{B}_{a+1} \stackrel{\iota}{\longrightarrow} \tilde{\mathscr{B}}_{a+1} \stackrel{\pi}{\longrightarrow} \mathscr{B}_{a}
$$

such that $\mathscr{B}_{a+1}=\left(\mathfrak{S}_{a+1}, \tilde{I}, R_{a}, \sigma, \tilde{\sigma}_{1}\right)$. This determines $\tilde{I}$ and $\tilde{\sigma}_{1}$ uniquely as follows. Let $\bar{J}^{a}=J^{a} / J^{a+1}$. We have $\widetilde{I}=E \mathfrak{S}_{a+1}+\bar{J}^{a}$ and $E \mathfrak{S}_{a+1} \cap \bar{J}^{a}=p \bar{J}^{a}$. The endomorphism $\bar{\sigma}$ of $\bar{J}^{a}$ induced by $\sigma$ is divisible by $p^{a}$, and the operator $\tilde{\sigma}_{1}: \tilde{I} \rightarrow \mathfrak{S}_{a+1}$ is the unique extension of $\sigma_{1}$ such that $\tilde{\sigma}_{1}(x)=p^{-1} \bar{\sigma}(x)$ for $x \in \bar{J}^{a}$. On the other hand, we consider the factorisation

$$
\mathscr{D}_{R_{a+1}} \stackrel{\iota^{\prime}}{\longrightarrow} \mathscr{D}_{R_{a+1} / R_{a}} \stackrel{\pi^{\prime}}{\longrightarrow} \mathscr{D}_{R_{a}}
$$

with respect to the trivial divided powers on the kernel $\mathfrak{m}_{R}^{a} / \mathfrak{m}_{R}^{a+1}$. Then $\varkappa_{a+1}$ is a u-homomorphism of frames $\tilde{\mathscr{B}}_{a+1} \rightarrow \mathscr{D}_{R_{a+1} / R_{a}}$. Indeed, the only condition to be verified is that for $x \in \bar{J}^{a}$ we have

$$
\tilde{\mathbb{T}}_{1}\left(\varkappa_{a+1}(x)\right)=u \cdot \varkappa_{a+1}\left(\tilde{\sigma}_{1}(x)\right)
$$

in the $k$-vector space $\widehat{W}\left(\mathfrak{m}_{R}^{a} / \mathfrak{m}_{R}^{a+1}\right)$. On this space $u$ acts as the identity. Let $y=\left(y_{0}, y_{1}, \ldots\right)$ in $W\left(\bar{J}^{a}\right)$ be defined by $y_{n}=\tilde{\sigma}_{1}^{n}(x)$. Then $\delta(x)=y$ because the Witt polynomials give $w_{n}(y)=p^{n} \tilde{\sigma}_{1}^{n}(x)=\sigma^{n}(x)=w_{n}(\delta(x))$ as required. Thus $\varkappa_{a+1}(x)$ is the reduction of $y$. Since $\tilde{\mathbb{F}}_{1}$ acts on $\widehat{W}\left(\mathfrak{m}_{R}^{a} / \mathfrak{m}_{R}^{a+1}\right)$ by a shift to the left, the relation (6-3) follows. We obtain compatible $u$-homomorphisms of frames $\varkappa_{*}:(6-1) \rightarrow(6-2)$. The homomorphisms $\pi$ and $\pi^{\prime}$ are crystalline; see the proof of [Lau 2010, Theorem 9.3]. Lifts of windows under $\iota$ and under $\iota^{\prime}$ are both classified by lifts of the Hodge filtration from $R_{a}$ to $R_{a+1}$ in a compatible way. Thus if $\varkappa_{a}$ is crystalline then so is $\varkappa_{a+1}$, and Theorem 6.6 follows by induction, using that $\varkappa_{1}$ is an isomorphism.

Following the terminology of [Vasiu and Zink 2010], a Breuil window relative to $\mathfrak{S} \rightarrow R$ is a pair $(Q, \phi)$ where $Q$ is a free $\mathfrak{S}$-module of finite rank and where $\phi: Q \rightarrow Q^{(\sigma)}$ is an S-linear map with cokernel annihilated by $E$. For $\operatorname{such}(Q, \phi)$ there is a unique linear map $\psi: Q^{(\sigma)} \rightarrow Q$ with $\psi \phi=E$; the pairs $(Q, \psi)$ are usually called Breuil-Kisin modules or Kisin modules. The category of $\mathscr{B}$-windows is equivalent to the category of Breuil windows relative to $\mathfrak{S} \rightarrow R$ by the assignment $\left(P, Q, F, F_{1}\right) \mapsto(Q, \phi)$, where $\phi$ is the composition of the inclusion $Q \rightarrow P$ with the inverse of $F_{1}^{\#}: Q^{(\sigma)} \cong P$; see [Lau 2010, Lemma 8.2].

Corollary 6.7. If the equivalent conditions of Proposition 6.2 hold, there is an equivalence of exact categories between p-divisible groups over $R$ and Breuil windows relative to $\mathfrak{S} \rightarrow R$.

Proof. This is analogous to [Lau 2010, Corollary 8.3], using Corollary 5.4. 
Following [Vasiu and Zink 2010] again, a Breuil module relative to $\mathfrak{S} \rightarrow R$ is a triple $(M, \phi, \psi)$ where $M$ is a finitely generated $\mathfrak{S}$-module annihilated by a power of $p$ and of projective dimension at most one and where $\phi: M \rightarrow M^{(\sigma)}$ and $\psi: M^{(\sigma)} \rightarrow M$ are $\mathfrak{S}$-linear maps with $\phi \psi=E$ and $\psi \phi=E$. If $R$ has characteristic zero, such triples are equivalent to pairs $(M, \phi)$ or $(M, \psi)$; see [Lau 2010, Lemma 8.6]. Again, the pairs $(M, \psi)$ are usually called Breuil-Kisin modules or Kisin modules.

Corollary 6.8. If the equivalent conditions of Proposition 6.2 hold, there is an equivalence of exact categories between commutative finite locally free group schemes of p-power order over $R$ and Breuil modules relative to $\mathfrak{S} \rightarrow R$.

Proof. This is analogous to [Lau 2010, Theorem 8.5].

Example 6.9. Let $R=W(k)$ and $\mathfrak{S}=W(k) \llbracket t \rrbracket$ with $\sigma(t)=t^{p}$. Define $\mathfrak{S} \rightarrow R$ by $t \mapsto p$; thus $E=p-t$. We have $\varkappa(E)=p-[p]$ and thus $u=v^{-1}(p-[p])$. Assume that $p=2$. Then $u=u_{0}$, and $\mathscr{B} \rightarrow \mathscr{D}_{R}$ is a strict frame homomorphism. This example has motivated the definition of Dieudonné displays for $p=2$.

\section{Breuil-Kisin modules and crystals}

We keep the notation of Section 6 and assume that the equivalent conditions of Proposition 6.2 hold. Assume that $R$ has characteristic zero. Let $S$ be the $p$-adic completion of the divided power envelope of the ideal $E \mathfrak{S} \subset \mathfrak{S}$, and let $I$ be the kernel of $S \rightarrow R$. Since $\sigma: \mathfrak{S} \rightarrow \mathfrak{S}$ preserves the ideal $(E, p)$, it extends to $\sigma: S \rightarrow S$. It is easy to see that $\sigma(I) \subseteq p S$, thus $\sigma: S \rightarrow S$ is a Frobenius lift again.

Proposition 7.1. Let $(Q, \phi)$ be a Breuil window relative to $\mathfrak{S} \rightarrow R$ and let $G$ be the associated p-divisible group over $R$; see Corollary 6.7. There is a natural isomorphism

$$
\mathbb{D}(G)_{S / R} \cong S \otimes_{\mathfrak{S}} Q^{(\sigma)}
$$

such that the Hodge filtration of $\mathbb{D}(G)_{S / R}$ corresponds to the submodule generated by $\phi(Q)+I Q^{(\sigma)}$, and the Frobenius of $\mathbb{D}(G)_{S / R}$ corresponds to the $\sigma$-linear endomorphism of $Q^{(\sigma)}$ defined by $x \mapsto 1 \otimes \phi^{-1}(E x)$.

In Kisin's theory (when $R$ is one-dimensional) the analogous result is immediate from the construction. To prove Proposition 7.1, we consider the frame

$$
\mathscr{S}=\left(S, I, R, \sigma, \sigma_{1}\right)
$$

with $\sigma_{1}(x)=\sigma(x) / p$ for $x \in I$. The inclusion $\mathfrak{S} \rightarrow S$ is a $u$-homomorphism of frames $\iota: \mathscr{B} \rightarrow \mathscr{S}$ with $u=\sigma(E) / p \in S$. This element is a unit as required, since the arrow $\mathfrak{S} \rightarrow R$ is mapped surjectively onto $W(k) \rightarrow k$, which gives a local homomorphism $S \rightarrow W(k)$ that maps $u$ to 1 . Recall that we have frames 
$\mathscr{D}_{R} \rightarrow \mathscr{D}_{R}^{+}$when $p=2$ and let us write $\mathscr{D}_{R}^{+}=\mathscr{D}_{R}$ when $p \geq 3$. Then we have the commutative diagram of frames

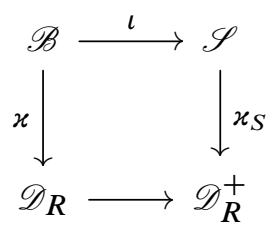

Indeed, since $\mathbb{W}^{+}(R) \rightarrow R$ is a divided power extension of $p$-adically complete rings, the ring homomorphism $\varkappa: \mathfrak{S} \rightarrow \mathbb{W}^{+}(R)$ extends to $\varkappa_{S}: S \rightarrow \mathbb{W}^{+}(R)$, which is a strict frame homomorphism $\mathscr{S} \rightarrow \mathscr{D}_{R}^{+}$. Here $\varkappa$ is crystalline by Theorem 6.6. The proof of Proposition 7.1 will use the following fact:

Theorem 7.2. The frame homomorphism $\varkappa_{S}$ is crystalline.

This is a variant of the main result of [Zink 2001b]. It is easy to see that $S$ is an admissible topological ring in the sense of Definition 1.2 if and only if $r=1$, i.e., if $R$ is a discrete valuation ring. In that case, the methods of [Zink 2001b] apply directly, but additional effort is needed to prove Theorem 7.2 in general. The proof is postponed to the next section.

Proof of Proposition 7.1. Let $\mathscr{P}_{0}=\left(P, Q, F, F_{1}\right)$ be the $\mathscr{B}$-window associated to $(Q, \phi)$; thus $P=Q^{(\sigma)}$, the inclusion map $Q \rightarrow P$ is $\phi$, and $F: P \rightarrow P$ is the $\sigma$-linear endomorphism of $Q^{(\sigma)}$ defined by $x \mapsto 1 \otimes \phi^{-1}(E x)$. By definition we have $\Phi_{R}(G)=\varkappa_{*}\left(\mathscr{P}_{0}\right)$, which implies that $\Phi_{R}^{+}(G)=\varkappa_{S *} \iota_{*}\left(\mathscr{P}_{0}\right)$; here we use Theorem 4.9 when $p=2$. On the other hand, the frames $\mathscr{S}$ and $\mathscr{D}_{R}^{+}$both satisfy the hypotheses of the beginning of Section 3C. Thus Construction 3.16 and Proposition 3.17 applied to $G$ give an $\mathscr{S}$-window $\mathscr{P}_{1}$ with an isomorphism $\varkappa_{S *}\left(\mathscr{P}_{1}\right) \cong \Phi_{R}^{+}(G)$, using the characterisation of $\Phi_{R}^{+}$in Theorem 3.19 and Proposition 3.23. Since the base change functor $\varkappa_{S *}$ is fully faithful by Theorem 7.2, the isomorphism $\varkappa_{S *}\left(\mathscr{P}_{1}\right) \cong \Phi_{R}^{+}(G) \cong \varkappa_{S *} \iota_{*}\left(\mathscr{P}_{0}\right)$ descends to an isomorphism $\mathscr{P}_{1} \cong \iota_{*}\left(\mathscr{P}_{0}\right)$, which proves the proposition.

7A. Proof of Theorem 7.2. Let us begin with a closer look on the $p$-adically complete ring $S$. For $m \geq 0$ let $S_{\langle m\rangle} \subseteq S$ be the closure of the $\mathfrak{S}$-algebra generated by $E^{i} / i$ ! for $i \leq p^{m}$.

Proposition 7.3. For $m \geq 1$, there is a surjective homomorphism of $\mathfrak{S}$-algebras $\mathfrak{S} \llbracket t_{1}, \ldots, t_{m} \rrbracket \rightarrow S_{\langle m\rangle}$ defined by $t_{i} \mapsto E^{p^{i}} / p^{i} !$.

In particular, $S_{\langle m\rangle}$ is a noetherian complete local ring.

Lemma 7.4. Let $A$ be a noetherian complete local ring with a descending sequence of ideals $A \supseteq \mathfrak{a}_{0} \supseteq \mathfrak{a}_{1} \supseteq \cdots$. Then $A \rightarrow \lim _{i} A / \mathfrak{a}_{i}$ is surjective. 
Proof. Let $\mathfrak{m}$ be the maximal ideal of $A$. For each $r$, the images of $\mathfrak{a}_{i} \rightarrow A / \mathfrak{m}^{r}$ stabilise for $i \rightarrow \infty$ to an ideal $\overline{\mathfrak{a}}_{r} \subseteq A / \mathfrak{m}^{r}$. We have

$$
\lim _{i} A / \mathfrak{a}_{i}=\lim _{i, r} A /\left(\mathfrak{a}_{i}+\mathfrak{m}^{r}\right)={\underset{\lim }{r}}_{r}\left(A / \mathfrak{m}^{r}\right) / \overline{\mathfrak{a}}_{r}
$$

Since the ideals $\overline{\mathfrak{a}}_{r}$ form a surjective system, taking the limit over $r$ of the exact sequences $0 \longrightarrow \overline{\mathfrak{a}}_{r} \longrightarrow A / \mathfrak{m}^{r} \longrightarrow\left(A / \mathfrak{m}^{r}\right) / \overline{\mathfrak{a}}_{r} \longrightarrow 0$ proves the lemma.

Proof of Proposition 7.3. Since the image of $E^{p^{i}} / p^{i}$ ! in $S / p^{n} S$ is nilpotent, there is a well-defined homomorphism $\pi_{m, n}: \mathfrak{S} \llbracket t_{1}, \ldots, t_{m} \rrbracket \rightarrow S / p^{n} S$ with $t_{i} \mapsto E^{p^{i}} / p^{i}$ !. By definition, $S_{\langle m\rangle}$ is the projective limit over $n$ of the image of $\pi_{n, m}$. The proposition follows by Lemma 7.4.

Let $K=W(k) \otimes \mathbb{Q}$ and $\mathfrak{S}_{\mathbb{Q}}=K \llbracket x_{1}, \ldots, x_{r} \rrbracket$. Since $\sigma: \mathfrak{S} \rightarrow \mathfrak{S}$ preserves the ideal $J=\left(x_{1}, \ldots, x_{r}\right)$, it extends to a homomorphism $\sigma: \mathfrak{S}_{\mathbb{Q}} \rightarrow \mathfrak{S}_{\mathbb{Q}}$. For $r=1$ it is easy to describe $S$ and $S_{\langle m\rangle}$ as explicit subrings of $\mathfrak{S}_{\mathbb{Q}}$, since instead of the divided powers of $E$ one can take the divided powers of $x_{1}^{e}$, where $e$ is defined by $p R=\mathfrak{m}_{R}^{e}$. For $r \geq 2$ the situation is more complicated.

Proposition 7.5. The natural embedding $\mathfrak{S} \rightarrow \mathfrak{S}_{\mathbb{Q}}$ extends to an injective homomorphism $S \rightarrow \mathfrak{S}_{\mathbb{Q}}$ that commutes with $\sigma$.

Thus $S_{\langle m\rangle}$ is the image of $\mathfrak{S} \llbracket t_{1}, \ldots, t_{m} \rrbracket \rightarrow \mathfrak{S}_{\mathbb{Q}}$ as in Proposition 7.3.

Proof of Proposition 7.5. Recall that $J=\left(x_{1}, \ldots, x_{r}\right)$ as an ideal of S. Choose $E^{\prime} \in J^{e}$ with $E-E^{\prime} \in p \mathfrak{S}$ such that $e$ is maximal; thus $p \in \mathfrak{m}_{R}^{e} \backslash \mathfrak{m}_{R}^{e+1}$. Let us write $\operatorname{gr}_{E^{\prime}}^{i}(\mathfrak{S})=E^{\prime i} \mathfrak{S} / E^{\prime i+1} \mathfrak{S}$, etc.

Lemma 7.6. The map of graded rings $\operatorname{gr}_{E^{\prime}}(\mathfrak{S}) \rightarrow \operatorname{gr}_{E^{\prime}}\left(\mathfrak{S}_{\mathbb{Q}}\right)$ is injective.

Proof. It suffices to show that $\mathfrak{S} / E^{\prime} \mathfrak{S} \rightarrow \mathfrak{S}_{\mathbb{Q}} / E^{\prime} \mathfrak{S}_{\mathbb{Q}}$ is injective. The choice of $E^{\prime}$ implies that the image of $E^{\prime}$ in the regular local rings $\mathfrak{S} / p \mathfrak{S}$ and $\mathfrak{S}_{\mathbb{Q}}$ lies in the same power of the maximal ideals. Therefore the $k$-dimension of $\mathfrak{S} /\left(p \mathfrak{S}+E^{\prime} \mathfrak{S}+J^{n}\right)$ is equal to the $K$-dimension of $\mathfrak{S}_{\mathbb{Q}} /\left(E^{\prime} \mathfrak{S}_{\mathbb{Q}}+J^{n} \mathfrak{S}_{\mathbb{Q}}\right)$. Since the last module is isomorphic to $\mathfrak{S} /\left(E^{\prime} \mathfrak{S}+J^{n}\right) \otimes \mathbb{Q}$, it follows that $\mathfrak{S} /\left(E^{\prime} \mathfrak{S}+J^{n}\right)$ is a free $W(k)$ module and injects into $\mathfrak{S}_{\mathbb{Q}} /\left(E^{\prime} \mathfrak{S}_{\mathbb{Q}}+J^{n} \mathfrak{S}_{\mathbb{Q}}\right)$. Since $\mathfrak{S} / E^{\prime} \mathfrak{S}$ and $\mathfrak{S}_{\mathbb{Q}} / E^{\prime} \mathfrak{S}_{\mathbb{Q}}$ are $J$-adically complete the lemma follows.

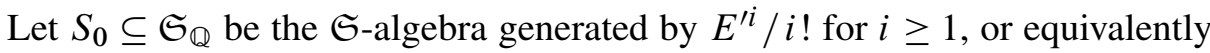
by $E^{i} / i$ ! for $i \geq 1$, so $S$ is the $p$-adic completion of $S_{0}$. Let $S_{0, n}$ be the image of $S_{0} \rightarrow \mathfrak{S}_{\mathbb{Q}} / E^{\prime n} \mathfrak{S}_{\mathbb{Q}}$ and let $\widetilde{S}=\lim _{n} S_{0, n}$. Each $S_{0, n}$ is a noetherian complete local ring with residue field $k$ and thus a $p$-adically complete ring. Since $S_{0, n}$ has no $p$-torsion it follows that $\widetilde{S}$ is $p$-adically complete. We obtain a homomorphism $S \rightarrow \widetilde{S} \subseteq \mathfrak{S}_{\mathbb{Q}}$ which extends $S_{0} \subseteq \widetilde{S} \subseteq \mathfrak{S}_{\mathbb{Q}}$.

Lemma 7.7. We have $S_{0} \cap p \widetilde{S}=p S_{0}$ inside $\widetilde{S}$. 
Proof. Let $x \in S_{0} \cap p \widetilde{S}$ be given. We have to show that $x$ lies in $p S_{0}$. Assume that $x \neq 0$ and choose an expression $(\star) x=\sum_{i=0}^{s} a_{i} E^{\prime n_{i}} / n_{i}$ ! with $a_{i} \in \mathfrak{S}$ such that $n_{0}<\cdots<n_{s}$. We use induction on $n_{s}-n_{0}$.

Suppose $E^{\prime}$ divides $a_{0}$ in $\mathfrak{S}$. Then $a_{0} E^{\prime n_{0}} / n_{0} !=a_{0}^{\prime} E^{\prime n_{0}^{\prime}} /\left(n_{0}^{\prime}\right)$ ! with $n_{0}^{\prime}=n_{0}+1$ and $a_{0}^{\prime}=n_{0}^{\prime} a_{0} / E^{\prime}$. If $s>0$ this allows us to find a new expression of $x$ of the type $(\star)$ with a smaller value of $n_{s}-n_{0}$, and we are done by induction. If $s=0$ we replace the expression $(\star)$ by $x=a_{0}^{\prime} E^{\prime n_{0}^{\prime}} / n_{0}^{\prime}$ !; call this a modification of the first type.

Suppose $E^{\prime}$ does not divide $a_{0}$ in $\mathfrak{S}$. Lemma 7.6 implies that the image of $x$ in $\operatorname{gr}_{E^{\prime}}^{n_{0}}\left(\mathfrak{S}_{\mathbb{Q}}\right)$ is nonzero. In $S_{0, n_{0}+1}$ we have $x=p y$. Choose an expression $y=$ $\sum_{i=\ell}^{n_{0}} c_{i} E^{\prime i} / i$ ! with $c_{i} \in \mathfrak{S}$ such that $\ell$ is maximal. Then $E^{\prime}$ does not divide $c_{\ell}$ in $\mathfrak{S}$, and Lemma 7.6 implies that $y$ has nonzero image in $\operatorname{gr}_{E^{\prime}}^{\ell}\left(\mathfrak{S}_{\mathbb{Q}}\right)$. Thus $\ell=n_{0}$. Using Lemma 7.6 again, it follows that the image of $a_{0}$ in $\mathfrak{S} / E^{\prime} \mathfrak{S}$ is divisible by $p$. Let $a_{0}=p b_{0}+b_{1} E^{\prime}$ with $b_{i} \in \mathfrak{S}$ and let $x^{\prime}=x-p b_{0} E^{\prime n_{0}} / n_{0}$ !. Then $x-x^{\prime} \in p S_{0}$; thus $x^{\prime} \in S_{0} \cap p \tilde{S}$, and we have to show that $x^{\prime} \in p S_{0}$. If $s>0$ we get an expression of $x^{\prime}$ of the type $(\star)$ with a smaller value of $n_{s}-n_{0}$, and we are done by induction. If $s=0$ we replace $x$ by $x^{\prime}$ and take for $(\star)$ the expression $x^{\prime}=a_{0}^{\prime} E^{\prime n_{0}^{\prime}} / n_{0}^{\prime}$ ! with $n_{0}^{\prime}=n_{0}+1$ and $a_{0}^{\prime}=n_{0}^{\prime} b_{1}$; call this a modification of the second type.

If $s>0$ the inductive step is already finished. So we may assume that $s=0$. We successively apply modifications of the first or second type depending on whether $E^{\prime}$ divides $a_{0}$. After at most $p$ steps, the new value of $a_{0}$ becomes divisible by $p$, and thus $x$ lies in $p S_{0}$.

Lemma 7.7 implies that $S_{0} / p^{n} S_{0} \rightarrow \widetilde{S} / p^{n} \tilde{S}$ is injective, so the projective limit $S \rightarrow \widetilde{S}$ is injective, and thus $S \rightarrow \mathfrak{S}_{\mathbb{Q}}$ is injective. In order that this map commutes with $\sigma$ it suffices to show that $S \rightarrow \mathfrak{S}_{\mathbb{Q}} / J^{n} \mathfrak{S}_{\mathbb{Q}}$ commutes with $\sigma$ for each $n$; this is true since $S_{0} \rightarrow \mathfrak{S}_{\mathbb{Q}} / J^{n} \mathfrak{S}_{\mathbb{Q}}$ commutes with $\sigma$, and the image of this map is $p$-adically complete. Thus Proposition 7.5 is proved.

We turn to the frames associated to the rings $S$ and $S_{\langle m\rangle}$.

Lemma 7.8. For $m \geq 1$ we have a subframe of $\mathscr{S}=\left(S, I, R, \sigma, \sigma_{1}\right)$,

$$
\mathscr{S}_{\langle m\rangle}=\left(S_{\langle m\rangle}, I_{\langle m\rangle}, R, \sigma, \sigma_{1}\right) .
$$

Proof. Necessarily $I_{\langle m\rangle}=I \cap S_{\langle m\rangle}$. We have to show that $\sigma: S \rightarrow S$ stabilises $S_{\langle m\rangle}$ and that $\sigma_{1}=p^{-1} \sigma: I \rightarrow S$ maps $I_{\langle m\rangle}$ into $S_{\langle m\rangle}$. We will show that $\sigma(S)$ and $\sigma_{1}(I)$ are even contained in $S_{\langle 1\rangle}$. Namely, we have $\sigma(E)=p x$ with $x \in \mathfrak{S}\left[E^{p} / p\right]$. Thus $\sigma_{1}\left(E^{i} / i !\right)=(p \cdot i !)^{-1}(p x)^{i}$ lies in $\mathfrak{S}\left[E^{p} / p\right]$, using that $1+v_{p}(i !) \leq i$ for $i \geq 1$. Since $I / p^{n} I$ is the kernel of $S / p^{n} S \rightarrow R / p^{n} R$, this ideal is generated as an $\mathfrak{S}$-module by the elements $E^{i} / i$ ! for $i \geq 1$. Thus the image of the map $I / p^{n+1} I \rightarrow S / p^{n} S$ induced by $\sigma_{1}$ lies in the image of $S_{\langle 1\rangle}$, and it follows that $\sigma_{1}(I) \subseteq S_{\langle 1\rangle}$. Since $S=\mathfrak{S}+I$, we get $\sigma(S) \subseteq S_{\langle 1\rangle}$.

Proposition 7.9. For $m \geq 1$ the inclusion $\mathscr{S}_{\langle m\rangle} \rightarrow \mathscr{S}$ is crystalline. 
Proof. This is a formal consequence of the relations $\sigma(S) \subseteq S_{\langle m\rangle}$ and $\sigma_{1}(I) \subseteq S_{\langle m\rangle}$ verified in the proof of Lemma 7.8.

Indeed, let $\mathscr{P}=\left(P, Q, F, F_{1}\right)$ be an $\mathscr{S}$-window. Choose a normal decomposition $P=L \oplus T$, and let $\Psi: L \oplus T \rightarrow P$ be the $\sigma$-linear isomorphism defined by $F_{1}$ on $L$ and by $F$ on $T$. Then $P_{\langle m\rangle}:=S_{\langle m\rangle} \Psi(L \oplus T)$ is a free $S_{\langle m\rangle}$-module with $S \otimes_{S_{\langle m\rangle}} P_{\langle m\rangle}=P$. Moreover, $F_{1}(Q) \subseteq P_{\langle m\rangle}$ and $F(P) \subseteq P_{\langle m\rangle}$. Let $Q_{\langle m\rangle}=Q \cap P_{\langle m\rangle}$. Then $P_{\langle m\rangle} / Q_{\langle m\rangle}=P / Q$ is a projective $R$-module. Let $P_{\langle m\rangle}=L_{\langle m\rangle} \oplus T_{\langle m\rangle}$ be a normal decomposition and let $\Psi_{\langle m\rangle}: L_{\langle m\rangle} \oplus T_{\langle m\rangle} \rightarrow P_{\langle m\rangle}$ be the $\sigma$-linear map defined by $F_{1}$ on $L_{\langle m\rangle}$ and by $F$ on $T_{\langle m\rangle}$. In order that the quadruple $\mathscr{P}_{\langle m\rangle}=\left(P_{\langle m\rangle}, Q_{\langle m\rangle}, F, F_{1}\right)$ is an $\mathscr{S}_{\langle m\rangle}$-window with base change $\mathscr{P}$ we need that the determinant of $\Psi_{\langle m\rangle}$ is invertible. But the determinant of $\Psi_{\langle m\rangle}$ becomes invertible in $S$ because $\mathscr{P}$ is a window, and $S_{\langle m\rangle} \rightarrow S$ is a local homomorphism. Thus the base change functor from $\mathscr{S}_{\langle m\rangle}$-windows to $\mathscr{S}$-windows is essentially surjective.

In order that the functor is fully faithful it suffices to show that it induces a bijection $\operatorname{End}\left(\mathscr{P}_{\langle m\rangle}\right) \rightarrow \operatorname{End}(\mathscr{P})$. Clearly the map is injective. We have to show that every $h \in \operatorname{End}(\mathscr{P})$ stabilises $P_{\langle m\rangle}$. But $h\left(F_{1}(Q)\right)=F_{1}(h(Q)) \subseteq F_{1}(Q) \subseteq P_{\langle m\rangle}$, and $F_{1}(Q)$ generates $P_{\langle m\rangle}$ as an $S_{\langle m\rangle}$-module. This proves the proposition.

Proposition 7.10. For $m \geq 1$, the composition $\mathscr{S}_{\langle m\rangle} \longrightarrow \mathscr{S}^{\varkappa_{S}} \longrightarrow \mathscr{D}_{R}^{+}$is crystalline.

This is the main step in the proof of Theorem 7.2. The proof of Proposition 7.10 is a variant of the proof of Theorem 6.6.

Proof. We choose $e$ such that $p \in \mathfrak{m}_{R}^{e} \backslash \mathfrak{m}_{R}^{e+1}$, and consider the index set $N=$ $\{1,2, \ldots\} \cup\{e+\}$, ordered by the natural order of $\mathbb{Z}$ and $e<e+<e+1$. For $n \in N$ let $n+\in N$ be its successor. Let $\mathfrak{m}_{R}^{e+}=\mathfrak{m}_{R}^{e+1}+p R$. For $n \in N$ let $R_{n}=R / \mathfrak{m}_{R}^{n}$. We equip the ideal $\mathfrak{m}_{R}^{n} / \mathfrak{m}_{R}^{n+}$ of $R_{n+}$ with the trivial divided powers if $n \neq e+$ and with the canonical divided powers of $p$ if $n=e+$; these are again trivial if $p$ is odd. In all cases the divided powers are compatible with the canonical divided powers of $p$, and we obtain frames

$$
\mathscr{D}_{R_{n+} / R_{n}}^{+}=\left(\mathbb{W}^{+}\left(R_{n+}\right), \square_{R_{n+} / R_{n}}^{+}, R_{n}, f, \tilde{f}_{1}\right) .
$$

Let $T_{n}$ be the image of $S_{\langle m\rangle} \stackrel{\varkappa_{S}}{\longrightarrow} \mathbb{W}^{+}(R) \longrightarrow \mathbb{W}^{+}\left(R_{n}\right)$. Since $\varkappa_{S} \sigma=f \varkappa_{S}$, the ring $T_{n}$ is stable under $f$. Let $K_{n}$ be the kernel of $T_{n} \rightarrow R_{n}$ and let $\widetilde{K}_{n}$ be the kernel of $T_{n+} \rightarrow R_{n}$.

We claim that $\tilde{f}_{1}\left(\widetilde{K}_{n}\right) \subseteq T_{n+}$.

To prove this, let $M_{n}$ be the kernel of $S_{\langle m\rangle} \rightarrow R_{n}$, so $\widetilde{K}_{n}$ is the image of $M_{n} \rightarrow$ $\mathbb{W}^{+}\left(R_{n+}\right)$. Since $\varkappa_{S} \sigma=f \varkappa_{S}$ and since $f_{1}$ is $f$-linear it suffices to show that a set of generators $x_{i}$ of the ideal $M_{n}$ with images $\varkappa_{S}\left(x_{i}\right)=\bar{x}_{i} \in \widetilde{K}_{n}$ satisfies $f_{1}\left(\bar{x}_{i}\right) \in$ $T_{n+}$. Since $\mathfrak{m}_{R}=J R$, for $n \neq e+$ the ideal $M_{n}$ is generated by $I_{\langle m\rangle}$ and $J^{n}$, while $M_{e+}$ is generated by $I_{\langle m\rangle}$ and $J^{e+1}$ and $p$. We check these generators case by case. 
First, for $x \in I_{\langle m\rangle}$ we have $f_{1}(\bar{x}) \in T_{n+}$ because $\mathscr{S}_{\langle m\rangle} \rightarrow \mathscr{D}_{R_{n+}}^{+}$is a frame homomorphism.

Assume that $n \neq e+$. The homomorphism $\delta: J^{n} / J^{n+1} \rightarrow W\left(J^{n} / J^{n+1}\right)$ is given by $\delta(x)=\left(x, \sigma_{1}(x),\left(\sigma_{1}\right)^{2}(x), \ldots\right)$. Indeed, applying the Witt polynomial $w_{n}$ to this equation gives $\sigma^{n}(x)=p^{n}\left(\sigma_{1}\right)^{n}(x)$, which is true. Since the divided powers on $\mathfrak{m}_{R}^{n} / \mathfrak{m}_{R}^{n+}$ are trivial, the endomorphism $\tilde{f}_{1}$ of $W\left(\mathfrak{m}_{R}^{n} / \mathfrak{m}_{R}^{n+}\right)$ is given by a shift to the left. Thus the map $\varkappa_{S}: J^{n} / J^{n+1} \rightarrow W\left(\mathfrak{m}_{R}^{n} / \mathfrak{m}_{R}^{n+}\right)$ satisfies $\varkappa_{S} \sigma_{1}=\tilde{f}_{1} \varkappa_{S}$, and we see that $f_{1}(\bar{x}) \in T_{n+}$ for $x \in J^{n}$.

Assume now that $n=e+$. Since $J^{e+1}$ maps to zero in $W\left(R_{e+1}\right)$, it remains to show that $\tilde{f}_{1}(p) \in T_{n+}$. Now $\log (p-v(1))=[p, 0,0, \ldots]$; see the proof of Lemma 1.9. Thus $\tilde{f}_{1}(p)=f_{1}(v(1))=1$, and the claim is proved.

We obtain frames

$$
\mathscr{T}_{n}=\left(T_{n}, K_{n}, R_{n}, f, f_{1}\right), \quad \mathscr{T}_{n+/ n}=\left(T_{n+}, \tilde{K}_{n}, R_{n}, f, \tilde{f}_{1}\right),
$$

and a commutative diagram of frames with strict homomorphisms

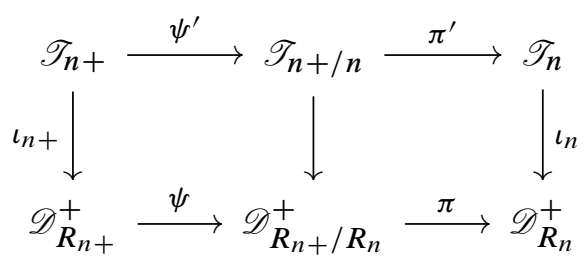

Here $\pi$ is crystalline because the hypotheses of Theorem 2.2 are satisfied; see the proof of Corollary 2.9. Since the vertical arrows are injective, it follows that $\pi^{\prime}$ satisfies the hypotheses of Theorem 2.2 as well, and thus $\pi^{\prime}$ is crystalline. Moreover, lifts of windows under $\psi$ and under $\psi^{\prime}$ correspond to lifts of the Hodge filtration from $R_{n}$ to $R_{n+}$ in the same way. Since $\iota_{1}$ is bijective, it follows that $\iota_{n}$ is crystalline for each $n$. Consider the limit frame

$$
\mathscr{T}=\lim _{n} \mathscr{T}_{n}=\left(T, K, R, f, f_{1}\right)
$$

The inclusion $\iota: \mathscr{T} \rightarrow \mathscr{D}_{R}^{+}$is the projective limit over $n$ of $\iota_{n}$ and thus crystalline. Since $S_{\langle m\rangle}$ is noetherian by Proposition 7.3, Lemma 7.4 implies that $T=\lim _{n} T_{n}$ is the image of $\varkappa_{S}: S_{\langle m\rangle} \rightarrow \mathbb{W}^{+}(R)$. If $\varkappa_{S}$ is injective, we get $\mathscr{S}_{\langle m\rangle} \overleftarrow{\leftarrow}_{\mathscr{T}}^{n}$, so $\mathscr{S}_{\langle m\rangle} \rightarrow \mathscr{D}_{R}^{+}$is crystalline as required.

Since we have not proved that $\varkappa_{S}$ is injective we need an extra argument. Let $\mathfrak{a}$ be the kernel of $\varkappa_{S}: S_{\langle m\rangle} \rightarrow \mathbb{W}^{+}(R)$ and let $\mathfrak{a}_{n}=\mathfrak{a} \cap J^{n} \mathfrak{S}_{\mathbb{Q}}$ for $n \geq 1$; here we use that $S$ is a subring of $\mathfrak{S}_{\mathbb{Q}}$ by Proposition 7.5. We have $\mathfrak{a}=\mathfrak{a}_{1}$. The ideals $\mathfrak{a}_{n}$ of $S_{\langle m\rangle}$ are stable under $\sigma$, and they are also stable under $\sigma_{1}$ since $S_{\langle m\rangle} / \mathfrak{a}$ and $\mathfrak{a}_{n} / \mathfrak{a}_{n+1}$ have no $p$-torsion. Thus we can define frames $\mathscr{S}_{\langle m\rangle, n}=\left(S_{\langle m\rangle} / \mathfrak{a}_{n}, I_{\langle m\rangle} / \mathfrak{a}_{n}, R, \sigma, \sigma_{1}\right)$. We have $\mathscr{S}_{\langle m\rangle, 1}=\mathscr{T}$, and the projective limit over $n$ of $\mathscr{S}_{\langle m\rangle, n}$ is isomorphic to $\mathscr{S}_{\langle m\rangle}$ 
by Lemma 7.4 and Proposition 7.5. The ideal $\mathfrak{a}_{n} / \mathfrak{a}_{n+1}$ is a finitely generated $W(k)$ submodule of $\left(J^{n} / J^{n+1}\right) \otimes \mathbb{Q}$. Since the conditions of Proposition 6.2 are satisfied, the endomorphism $\sigma_{1}$ of $\mathfrak{a}_{n} / \mathfrak{a}_{n+1}$ is $p$-adically nilpotent. Thus $\mathscr{S}_{\langle m\rangle, n+1} \rightarrow \mathscr{S}_{\langle m\rangle, n}$ is crystalline; see the proof of [Lau 2010, Theorem 9.3]. It follows that $\mathscr{S}_{\langle m\rangle} \rightarrow \mathscr{T}$ is crystalline, so $\mathscr{S}_{\langle m\rangle} \rightarrow \mathscr{D}_{R}^{+}$is crystalline too.

Theorem 7.2 follows from Propositions 7.9 and 7.10.

Remark 7.11. Assume that $r=1$; i.e., $R$ is a discrete valuation ring. If $p R=\mathfrak{m}_{R}^{e}$, the ring $S$ is the $p$-adic completion of $W(k) \llbracket t \rrbracket\left[\left\{t^{e m} / m !\right\}_{m \geq 1}\right]$. It is easy to see that each quotient $S / p^{n} S$ is admissible, so the $p$-adically complete ring $S$ is an admissible topological ring. In particular, $\mathbb{W}^{+}(S)$ is defined. Since we assumed that the image of $\delta: \mathfrak{S} \rightarrow W(\mathfrak{S})$ lies in $\mathbb{W}(\mathfrak{S})$, it follows that the image of $\delta: S \rightarrow W(S)$ lies in $\mathbb{W}^{+}(S)$, using that $\mathbb{W}^{+}(S) \rightarrow R$ is the projective limit of the divided power extensions $\mathbb{W}^{+}\left(S / p^{n} S\right) \rightarrow R / p^{n} R$ and that each $\mathbb{W}^{+}\left(S / p^{n} S\right)$ is $p$-adically complete. If $p$ is odd this means that $\mathscr{S}$ is a Dieudonné frame in the sense of [Zink 2001b, Definition 3.1], and Theorem 7.2 becomes a special case of [Zink 2001b, Theorem 3.2]. For $p=2$ the proof of [loc. cit.] works as well. The starting point is the construction of an inverse functor of $\varkappa_{S *}$; it maps a $\mathscr{D}_{R}^{+}$-window $\mathscr{P}$ to the value of its crystal $\mathbb{D}^{+}(\mathscr{P})_{S / R}$, equipped with an appropriate $\mathscr{S}$-window structure.

If $r \geq 2$, the ring $S$ is not admissible and thus the crystal of a $\mathscr{D}_{R}^{+}$-window can not be evaluated at $S / R$. However, one can define by hand a subframe $\mathscr{D}_{S / R}^{+}$of $\mathscr{W}_{S / R}$ such that $\mathscr{D}_{S / R}^{+} \rightarrow \mathscr{D}_{R}^{+}$is crystalline. This allows us to evaluate the crystal at $S / R$ and to define an inverse functor of $\varkappa_{S *}$ as before. The underlying ring of $\mathscr{D}_{S / R}^{+}$is defined as follows. Let $S_{m, n}$ be the image of $S_{\langle m\rangle} \rightarrow S / p^{n} S$ and let $I_{m, n}$ be the kernel of $S_{m, n} \rightarrow R / p^{n} R$. The divided Witt polynomials define an isomorphism $\log : W\left(I / p^{n} I\right) \cong\left(I / p^{n} I\right)^{\mathbb{N}}$, and our ring is $\lim _{n} \lim _{\longrightarrow}$ of the rings $\mathbb{W}^{+}\left(S_{0, n}\right)+\log ^{-1}\left(\left(I_{m, n}\right)^{\langle\mathbb{N}\rangle}\right)$. The $\lim _{n}$ of these rings for fixed $m \geq 1$ gives a frame $\mathscr{D}_{S_{\langle m\rangle} / R}^{+}$with a crystalline homomorphism to $\mathscr{D}_{R}^{+}$. This allows us to construct the inverse functor from $\mathscr{D}_{R}^{+}$-windows to $\mathscr{S}_{\langle m\rangle}$-windows. We leave out the details.

\section{Rigidity of $p$-divisible groups}

In this section, we record a rigidity property of the category of $p$-divisible groups that will be used in Section 9. As preparation, for a local ring $R$ we consider the additive category $\mathcal{F}_{R}$ of commutative finite locally free $p$-group schemes over $R$. It is known that $\mathcal{F}_{R}$ is equivalent to the full subcategory of the bounded derived category of the exact category of $p$-divisible groups over $R$ formed by the complexes of length one which are isogenies; see [Kisin 2006, (2.3.5)]. In elementary terms this equivalence can be expressed as follows: 
Proposition 8.1. For a local ring $R$, let $\mathcal{I}_{R}$ be the category with isogenies of p-divisible groups over $R$ as objects and homomorphisms of complexes modulo homotopy as homomorphisms. The set $S$ of quasi-isomorphisms in $\mathcal{I}_{R}$ allows a calculus of right fractions. In particular, the localised category $S^{-1} \mathcal{I}_{R}$ is additive. It is equivalent to the additive category $\mathcal{F}_{R}$.

For completeness let us prove this directly.

Proof. Let $\tilde{\mathcal{I}}_{R}$ be the category with isogenies of $p$-divisible groups over $R$ as objects and homomorphisms of complexes as homomorphisms. We denote isogenies by $X=\left[X^{0} \rightarrow X^{1}\right]$. Let $h^{0}(X)$ be the kernel of $X^{0} \rightarrow X^{1}$. A homomorphism $f: X \rightarrow Y$ in $\widetilde{\mathcal{I}}_{R}$ is homotopic to zero if and only if $h^{0}(f)$ is the zero map; the homotopy is unique if it exists. We claim:

( $\star$ For each homomorphism $f: X \rightarrow Y$ in $\widetilde{\mathcal{I}}_{R}$, one can find a quasi-isomorphism $t: Z \rightarrow X$ in $\tilde{\mathcal{I}}_{R}$ and a homomorphism $g: Z \rightarrow Y$ in $\tilde{\mathcal{I}}_{R}$ which is an epimorphism in both components such that $f t$ is homotopic to $g$. Namely, embed $h^{0}(X)$ into $Z^{0}=X^{0} \oplus Y^{0}$ by $(1, f)$ and put $Z^{1}=Z^{0} / h^{0}(X)$. Define $t$ and $g$ by the projections $Z^{0} \rightarrow X^{0}$ and $Z^{0} \rightarrow Y^{0}$. There is a homotopy between $f t$ and $g$ because $f t=g$ on $h^{0}(Z)$, and $(\star)$ is proved.

Next, for given homomorphisms $X \stackrel{f}{\longrightarrow} Y \stackrel{s}{\longleftarrow} Y^{\prime}$ in $\widetilde{\mathcal{I}}_{R}$, where $s$ is a quasiisomorphism, one can find an isogeny $X^{\prime}$ with a homomorphism $g: X^{\prime} \rightarrow Y^{\prime}$ and a quasi-isomorphism $t: X^{\prime} \rightarrow X$ such that $f t$ is homotopic to $s g$. Indeed, by $(\star)$ we can assume that the components of $f$ are epimorphisms. Then take $X^{\prime}=X \times_{Y} Y^{\prime}$ componentwise. It follows easily that $S$ allows a calculus of right fractions. We have an additive functor $h^{0}: S^{-1} \mathcal{I} \rightarrow \mathcal{F}$. It is surjective on isomorphism classes by a theorem of Raynaud [Berthelot et al. 1982, Théorème 3.1.1]. Let $X$ and $Y$ be isogenies. The functor $h^{0}$ is full, because for a given homomorphism $f_{0}: h^{0}(X) \rightarrow$ $h^{0}(Y)$, the construction in $(\star)$ allows us to represent $f_{0}$ as $h^{0}\left(g t^{-1}\right)$. The functor is faithful because if a right fraction $g t^{-1}: X \rightarrow Y$ induces zero on $h^{0}$ then $g$ induces zero on $h^{0}$, and thus $g$ is homotopic to zero.

Let (Art) be the category of local Artin schemes with perfect residue field of characteristic $p$, and let $(p$-div) $\rightarrow$ (Art) be the fibred category of $p$-divisible groups over schemes in (Art).

Lemma 8.2. Assume that $u$ is an exact automorphism of the fibred category ( $p$-div) over (Art) such that for the group $E=\mathbb{Q}_{p} / \mathbb{Z}_{p}$ over Spec $\mathbb{F}_{p}$ there is an isomorphism $u(E) \cong E$. Then $u$ is isomorphic to the identity functor.

Proof. For each $U$ in (Art) we are given a functor $G \mapsto G^{u}$ from the category of $p$ divisible groups over $U$ to itself, which preserves short exact sequences, compatible with base change in $U$, such that $\operatorname{Hom}(G, H) \cong \operatorname{Hom}\left(G^{u}, H^{u}\right)$. We have to show that there is an natural isomorphism $G^{u} \cong G$ for all $G$, compatible with base change 
in $U$. Let ( $p$-fin) $\rightarrow$ (Art) be the fibred category of commutative finite locally free $p$-group schemes over schemes in (Art). By Proposition 8.1, $u$ induces an automorphism of ( $p$-fin) over (Art). Let $H \in(p$-fin) over $U \in$ (Art) be given. Assume that $p^{n}$ annihilates $H$ and $H^{u}$. For each $T \rightarrow U$ in (Art) there is a natural isomorphism

$$
H(T) \cong \operatorname{Hom}_{T}\left(\mathbb{Z} / p^{n} \mathbb{Z}, H_{T}\right) \cong \operatorname{Hom}_{T}\left(\mathbb{Z} / p^{n} \mathbb{Z}, H_{T}^{u}\right) \cong H^{u}(T),
$$

using that $\left(\mathbb{Z} / p^{n} \mathbb{Z}\right)^{u} \cong \mathbb{Z} / p^{n} \mathbb{Z}$. Since commutative finite locally free group schemes over $U$ form a full subcategory of the category of abelian presheaves on (Art) $/ U$, we get a natural isomorphism $H \cong H^{u}$, which induces a natural isomorphism $G \cong G^{u}$ for all $p$-divisible groups $G$ over $U$.

\section{The reverse functor}

We fix an admissible ring $R$ which is local of dimension zero; thus $k=R_{\text {red }}$ is a perfect field of characteristic $p$. In this case, one can write down an inverse of the functor $\Phi_{R}$ as follows. The construction appears in [Lau 2009] when $p \geq 3$ or $p R=0$ and extends to the general case with appropriate changes.

Definition 9.1. Let $\mathcal{J}_{R}$ be the category of $R$-algebras $A$ such that $\mathcal{N}_{A}$ is bounded nilpotent and $A_{\text {red }}$ is the union of finite dimensional $k$-algebras.

We call a ring homomorphism $A \rightarrow B$ ind-étale if $B$ is the filtered direct limit of étale $A$-algebras.

Lemma 9.2. Every $A \in \mathcal{J}_{R}$ is admissible. The category $\mathcal{J}_{R}$ is stable under tensor products. If $A \rightarrow B$ is an ind-étale or a quasi-finite ring homomorphism with $A \in \mathcal{J}_{R}$ then $B \in \mathcal{J}_{R}$.

Proof. Since a reduced finite $k$-algebra is étale and thus perfect, every $A$ in $\mathcal{J}_{R}$ is admissible. Let $A \rightarrow B$ a ring homomorphism with $A \in \mathcal{J}_{R}$. Then $\mathcal{N}_{A} B$ is bounded nilpotent, so $B$ is lies in $\mathcal{J}_{R}$ if and only if $B / \mathcal{N}_{A} B$ lies in $\mathcal{J}_{R}$. For given homomorphisms $B \leftarrow A \rightarrow C$ in $\mathcal{J}_{R}$ we have to show that $B \otimes_{A} C$ lies in $\mathcal{J}_{R}$. By the preceding remark, we may assume that $A, B, C$ are reduced. Then $B \otimes_{A} C$ is the direct limit of étale $k$-algebras and thus lies in $\mathcal{J}_{R}$. Let $g: A \rightarrow B$ be an ind-étale or quasi-finite ring homomorphism with $A \in \mathcal{J}_{R}$. In order to show that $B \in \mathcal{J}_{R}$ we may assume that $A$ is reduced. Then every finitely generated $k$-subalgebra of $A$ is étale. Thus each étale $A$-algebra is defined over an étale $k$-subalgebra of $A$. If $g$ is ind-étale it follows that $B$ lies in $\mathcal{J}_{R}$. Assume that $g$ is quasi-finite. Then $B$ is defined over an étale $k$-subalgebra of $A$. Since all finite $k$-algebras lie in $\mathcal{J}_{R}$ and since $\mathcal{J}_{R}$ is stable under tensor products, it follows that $B \in \mathcal{J}_{R}$.

Let $S=\operatorname{Spec} R$ and let $\mathcal{J}_{S}$ be the category of affine $S$-schemes $\operatorname{Spec} A$ with $A \in \mathcal{J}_{R}$. If $\tau$ is either ind-étale or fpqc, we consider the $\tau$-topology on $\mathcal{J}_{S}$ in which coverings are finite families of morphisms ( $\operatorname{Spec} B_{i} \rightarrow \operatorname{Spec} A$ ) such that 
the associated homomorphism $A \rightarrow \prod_{i} B_{i}$ is faithfully flat, and ind-étale if $\tau$ is ind-étale. Let $\widetilde{S}_{\mathcal{J}, \tau}$ be the category of $\tau$-sheaves on $\mathcal{J}_{S}$.

Lemma 9.3. The presheaf of rings $\mathbb{W}$ on $\mathcal{J}_{S}$ is an fpqc sheaf.

Proof. See [Lau 2009, Lemma 1.5]. Since the presheaf $W$ is an fpqc sheaf, it suffices to show that for an injective ring homomorphism $A \rightarrow B$ in $\mathcal{J}_{R}$ we have $\mathbb{W}(A)=\mathbb{W}(B) \cap W(A)$ in $W(B)$. This is easily verified using that $A_{\text {red }} \rightarrow B_{\text {red }}$ is injective and $\widehat{W}\left(\mathcal{N}_{A}\right)=\widehat{W}\left(\mathcal{N}_{B}\right) \cap W(A)$ in $W\left(\mathcal{N}_{B}\right)$.

Let $\mathscr{P}$ be a Dieudonné display over $R$. For $\operatorname{Spec} A \in \mathcal{J}_{S}$ let $\mathscr{P}_{A}=\left(P_{A}, Q_{A}, F, F_{1}\right)$ be the base change of $\mathscr{P}$ to $A$. We define two complexes $Z(\mathscr{P})$ and $Z^{\prime}(\mathscr{P})$ of presheaves of abelian groups on $\mathcal{J}_{S}$ by

$$
\begin{aligned}
Z(\mathscr{P})(\operatorname{Spec} A) & =\left[Q_{A} \stackrel{F_{1}-1}{\longrightarrow} P_{A}\right], \\
Z^{\prime}(\mathscr{P})(\operatorname{Spec} A) & =\left[Q_{A} \stackrel{F_{1}-1}{\longrightarrow} P_{A}\right] \otimes[\mathbb{Z} \rightarrow \mathbb{Z}[1 / p]],
\end{aligned}
$$

such that $Z(\mathscr{P})$ lies in degrees 0,1 and $Z^{\prime}(\mathscr{P})$ lies in degrees $-1,0,1$, so the second tensor factor lies in degrees $-1,0$.

Proposition 9.4. The components of $Z^{\prime}(\mathscr{P})$ are fpqc sheaves on $\mathcal{J}_{S}$. The ind-étale (and thus the fpqc) cohomology sheaves of $Z^{\prime}(\mathscr{P})$ vanish outside degree zero, and the cohomology sheaf in degree zero is represented by a well-defined p-divisible group $\mathrm{BT}_{R}(\mathscr{P})$ over $R$. This defines an additive and exact functor

$\mathrm{BT}_{R}:($ Dieudonné displays over $R) \rightarrow(p$-divisible groups over $R)$.

One can also express the definition of the functor $\mathrm{BT}_{R}$ by the formula

$$
\mathrm{BT}_{R}(\mathscr{P})=\left[Q \stackrel{F_{1}-1}{\longrightarrow} P\right] \otimes \otimes^{L} \mathbb{Q}_{p} / \mathbb{Z}_{p}
$$

in the derived category of either ind-étale or fpqc abelian sheaves on $\mathcal{J}_{S}$.

Proof. This is essentially proved in [Lau 2009], but we recall the arguments for completeness and because there is a small modification when $p=2$. To begin with, $p$-divisible groups over $R$ form a full subcategory of the abelian presheaves on $\mathcal{J}_{S}$ because finite group schemes over $R$ lie in $\mathcal{J}_{S}$; see Lemma 9.2. Hence $\mathrm{BT}_{R}$ is a well-defined additive functor if the assertions on the cohomology of $Z^{\prime}(\mathscr{P})$ hold. Since an exact sequence of Dieudonné displays over $R$ induces an exact sequence of the associated complexes of presheaves $Z^{\prime}$, the functor $\mathrm{BT}_{R}$ is exact if it is defined.

The components of $Z(\mathscr{P})$ and $Z^{\prime}(\mathscr{P})$ are fpqc sheaves on $\mathcal{J}_{S}$ by Lemma 9.3. These complexes carry two filtrations. First, a Dieudonné display is called étale if $Q=P$, and nilpotent if $V^{\#}$ is topologically nilpotent. Every Dieudonné display over $R$ is naturally an extension of an étale by a nilpotent Dieudonné display, which induces exact sequences of the associated complexes $Z(\ldots)$ and $Z^{\prime}(\ldots)$. Thus we 
may assume that $\mathscr{P}$ is étale or nilpotent. Second, for every $\mathscr{P}$ we have an exact sequence of complexes of presheaves

$$
0 \longrightarrow \hat{Z}(\mathscr{P}) \longrightarrow Z(\mathscr{P}) \longrightarrow Z_{\text {red }}(\mathscr{P}) \longrightarrow 0,
$$

defined by $Z_{\text {red }}(\mathscr{P})(\operatorname{Spec} A)=Z(\mathscr{P})\left(\operatorname{Spec} A_{\text {red }}\right)$. The same holds for $Z^{\prime}$ instead of $Z$. We write $\hat{Z}(\mathscr{P})=[\widehat{Q} \rightarrow \widehat{P}]$.

Assume that $\mathscr{P}$ is étale. Then $F_{1}: P \rightarrow P$ is an $f$-linear isomorphism. Thus $F_{1}: \hat{P} \rightarrow \widehat{P}$ is elementwise nilpotent, and the complex $\widehat{Z}(\mathscr{P})$ is acyclic. It follows that $Z(\mathscr{P})$ is quasi-isomorphic to the complex $Z_{\text {red }}(\mathscr{P})=Z_{\text {red }}$, which is the projective limit of the complexes $Z_{\text {red, } n}=Z_{\text {red }} / p^{n} Z_{\text {red. }}$. In the étale topology, each $Z_{\text {red }, n}$ is a surjective homomorphism of sheaves whose kernel is a locally constant sheaf $G_{n}$ of free $\mathbb{Z} / p^{n} \mathbb{Z}$-modules of rank equal to the rank of $P$. The system $\left(G_{n}\right)_{n}$ defines an étale $p$-divisible group $G$ over $R$, and $Z_{\text {red }}$ is quasi-isomorphic to $T_{p} G=\lim _{\leftarrow} G_{n}$ as ind-étale sheaves. It follows that $Z^{\prime}(\mathscr{P}) \simeq Z_{\text {red }}^{\prime}(\mathscr{P})$ is quasiisomorphic to the complex $\left[T_{p} G \rightarrow T_{p} G \otimes \mathbb{Z}[1 / p]\right]$ in degrees $-1,0$, which is quasi-isomorphic to $G$ in degree zero (as ind-étale sheaves).

Assume that $\mathscr{P}$ is nilpotent. Then the complex $Z_{\text {red }}(\mathscr{P})$ is acyclic because its value over Spec $A$ is isomorphic to [1-V: $P_{A_{\text {red }}} \rightarrow P_{A_{\text {red }}}$, where $V$ is a topologically nilpotent $f^{-1}$-linear homomorphism. Thus $Z(\mathscr{P})$ is quasi-isomorphic to $\hat{Z}(P)$. To $\mathscr{P}$ we associate a nilpotent display by the $u_{0}$-homomorphism of frames $\mathscr{D}_{R} \rightarrow \mathscr{W}_{R}$. By [Zink 2002, Theorem 81 and Corollary 89 ] there is a formal $p$-divisible group $G$ over $R$ associated to this display such that for each $A \in \mathcal{J}_{R}$ there is an exact sequence

$$
0 \longrightarrow \hat{Q}(A) \stackrel{u_{0} F_{1}-1}{\longrightarrow} \hat{P}(A) \longrightarrow G(A) \longrightarrow 0 ;
$$

this is the direct limit of the corresponding sequences for the finitely generated (nilpotent) subalgebras of $\mathcal{N}_{A}$. Since $u_{0} \in W\left(\mathbb{Z}_{p}\right)$ maps to 1 in $W\left(\mathbb{F}_{p}\right)$, there is a unique $c \in W\left(\mathbb{Z}_{p}\right)$ which maps to 1 in $W\left(\mathbb{F}_{p}\right)$ such that $u_{0}=c f\left(c^{-1}\right)$, namely $c=u_{0} f\left(u_{0}\right) f^{2}\left(u_{0}\right) \cdots$. Multiplication by $c$ in both components defines an isomorphism of complexes

$$
\left[\widehat{Q}(A) \stackrel{F_{1}-1}{\longrightarrow} \widehat{P}(A)\right] \cong\left[\widehat{Q}(A) \stackrel{u_{0} F_{1}-1}{\longrightarrow} \widehat{P}(A)\right]
$$

It follows that $Z^{\prime}(\mathscr{P}) \simeq \hat{Z}^{\prime}(\mathscr{P})$ is quasi-isomorphic to $G$ in degree zero.

Remark 9.5. Recall that $\mathscr{D}_{R}=\left(\mathbb{W}(R), \rrbracket_{R}, f, \mathbb{\Vdash}_{1}\right)$ is viewed as a Dieudonné display over $R$. We have $\mathrm{BT}_{R}\left(\mathscr{D}_{R}\right)=\mu_{p} \infty$ by [Zink 2002, (211)].

Lemma 9.6. Let $R \rightarrow R^{\prime}$ be a homomorphism of admissible rings which are local of dimension zero. For each Dieudonné display $\mathscr{P}$ over $R$ there is a natural isomorphism

$$
\mathrm{BT}_{R}(\mathscr{P})_{R^{\prime}} \cong \mathrm{BT}_{R^{\prime}}\left(\mathscr{P}_{R^{\prime}}\right)
$$


Proof. If the residue field of $R^{\prime}$ is an algebraic extension of $k$, every ring in $\mathcal{J}_{R^{\prime}}$ lies in $\mathcal{J}_{R}$, and the assertion follows directly from the construction of $\mathrm{BT}_{R}$. In general, let $\mathscr{E}_{R}$ be the category of all $R$-algebras which are admissible rings, and let $\mathscr{E}_{S}$ be the category of affine $S$-schemes $\operatorname{Spec} A$ with $A \in \mathscr{E}_{R}$, endowed with the ind-étale topology. The complexes of presheaves $Z(\mathscr{P})$ and $Z^{\prime}(\mathscr{P})$ on $\mathcal{J}_{S}$ defined in (9-1) and (9-2) extend to complexes of presheaves on $\mathscr{E}_{S}$ defined by the same formulas. The proof of Lemma 9.2 shows that for an ind-étale ring homomorphism $A \rightarrow B$ with $A \in \mathscr{E}_{R}$ we have $B \in \mathscr{E}_{R}$ as well. Using this, the proof of Proposition 9.4 shows that the ind-étale cohomology sheaves of $Z^{\prime}(\mathscr{P})$ on $\mathscr{E}_{S}$ vanish outside degree zero, and $H^{0}\left(Z^{\prime}(\mathscr{P})\right)$ is naturally isomorphic to $\mathrm{BT}_{R}(\mathscr{P})$ as a sheaf on $\mathscr{E}_{S}$. Since every ring in $\mathscr{E}_{R^{\prime}}$ lies in $\mathscr{E}_{R}$, the lemma follows as in the first case.

Proposition 9.7. The functor $\mathrm{BT}_{R}$ is an equivalence of exact categories which is a quasi-inverse of the functor $\Phi_{R}$.

Proof. By Section 3A we may assume that $R$ is a local Artin ring. Since $p$-divisible groups and Dieudonné displays over $k$ have universal deformation rings which are power series rings over $W(k)$, once the functor $\mathrm{BT}_{R}$ is defined, in order to show that it is an equivalence of categories it suffices to consider the cases $R=k$ and $R=k[\varepsilon]$. In particular, if $p=2$, we may assume that $p R=0$, so that the results of [Zink 2001a] and [Lau 2009] can be applied. The category $\mathcal{C}_{R}$ used in [Lau 2009] is the category of all $A \in \mathcal{J}_{R}$ such that $\mathcal{N}_{A}$ is nilpotent. Since this subcategory is stable under ind-étale extensions, it does not make a difference whether the functor $\mathrm{BT}_{R}$ is defined in terms of $\mathcal{C}_{R}$ or $\mathcal{J}_{R}$. Thus $\mathrm{BT}_{R}$ is an equivalence by [Lau 2009, Theorem 1.7], which relies on the equivalence proved in [Zink 2001a]. It is easily verified that $\mathrm{BT}_{R}\left(\Phi_{R}\left(\mathbb{Q}_{p} / \mathbb{Z}_{p}\right)\right)$ is isomorphic to $\mathbb{Q}_{p} / \mathbb{Z}_{p}$. Thus $\mathrm{BT}_{R}$ is a quasi-inverse of $\Phi_{R}$ by Lemmas 8.2 and 9.6. It is easily verified that the functors $\mathrm{BT}_{R}$ and $\Phi_{R}$ preserve short exact sequences.

\section{Appendix: PD envelopes of regular immersions}

This section provides a reference for the flatness of the divided power envelope of a regular immersion, which is used in the proof of Lemma 1.13. Let us recall regular immersions following [SGA $1971, \mathrm{VII}$ ]. For a ring $A$, a projective $A$-module $M$ of finite type, and a linear map $f: M \rightarrow A$, one defines the Koszul complex

$$
K_{*}(A, f)=\left[\cdots \rightarrow \Lambda^{2} M \rightarrow \Lambda^{1} M \rightarrow A\right]
$$

with differential given by $x_{1} \wedge \cdots \wedge x_{n} \mapsto \sum(-1)^{i+1} f\left(x_{i}\right) x_{1} \wedge \cdots \hat{x}_{i} \cdots \wedge x_{n}$. Let $I=f(M) \subseteq A$. One calls $f$ regular if the augmentation $K_{*}(A, f) \rightarrow A / I$ is a quasi-isomorphism. If $x_{1}, \ldots, x_{r}$ is a regular sequence in $A$ and $f: A^{r} \rightarrow A$ is given by $f(a)=\sum a_{i} x_{i}$, then $f$ is regular in the previous sense. For a ring homomorphism $A \rightarrow A^{\prime}$, let $f^{\prime}: M^{\prime} \rightarrow A^{\prime}$ be the scalar extension of $f$, and let 
$I^{\prime}=f^{\prime}\left(M^{\prime}\right)$. If both $f$ and $f^{\prime}$ are regular, then $\operatorname{Tor}_{i}^{A}\left(A / I, A^{\prime}\right)=0$ for $i \geq 1$ and thus $I^{\prime}=I \otimes_{A} A^{\prime}$. A closed immersion of schemes $Y \rightarrow X$ is called regular if locally in $X$ it takes the form $\operatorname{Spec} A / I \rightarrow \operatorname{Spec} A$, where $I=f(M)$ for a regular homomorphism $f: M \rightarrow A$.

Proposition A.1. Let $S$ be a scheme and $i: Y \rightarrow X$ be a regular closed immersion of flat $S$-schemes. Then the divided power envelope $\mathscr{D}_{X}(Y)$ is flat over $S$.

Under additional hypotheses, this is proved in [Berthelot et al. 1982, Lemme 2.3.3]. We use the following description of the divided power polynomial algebra:

Lemma A.2. For a ring $R$, let $A_{0}=R\left[T_{1}, \ldots, T_{n}\right]$, and let $B_{0}=R\left\langle T_{1}, \ldots, T_{n}\right\rangle$ be the divided power envelope of $I_{0}=\left(T_{1}, \ldots, T_{n}\right) \subseteq A_{0}$. Then one can write $B_{0}=\lim _{\rightarrow} M_{0, r}$ as an $A_{0}$-module, the direct limit taken over $r \in \mathbb{N}$ ordered multiplicatively, such that there are exact sequences of $A_{0}$-modules

$$
0 \longrightarrow J_{0, r} \longrightarrow M_{0, r} \longrightarrow N_{0, r} \longrightarrow 0
$$

with $J_{0, r}=\left(T_{1}^{r}, \ldots, T_{n}^{r}\right)$ and where $N_{0, r}$ has a finite filtration with quotients isomorphic to $A_{0} / I_{0}=R$.

Proof. The assertion is stable under base change in $R$, so we may take $R=\mathbb{Z}$. Then $B_{0}$ is the $A_{0}$-subalgebra of $A_{0} \otimes \mathbb{Q}$ generated by all $T_{i}^{m} / m !$. Let $M_{0, r}=B_{0} \cap r^{-1} A_{0}$ inside $A_{0} \otimes \mathbb{Q}$. Then $r^{-1} J_{0, r}$ is contained in $M_{0, r}$, and the quotient $N_{0, r}$ coincides with the image of $M_{0, r}$ in $\left(A_{0} / J_{0, r}\right) \otimes \mathbb{Q}$. Any maximal filtration of the latter by monomial ideals gives the required filtration of $N_{0, r}$.

Proof of Proposition A.1. We may assume that $S=\operatorname{Spec} R, X=\operatorname{Spec} A$ and $Y=\operatorname{Spec} A / I$, where $I$ is the image of a regular map $f: A^{r} \rightarrow A$. We have $f(a)=\sum a_{i} x_{i}$ for a sequence $x_{1}, \ldots, x_{r}$ in $A$. Let $A_{0}=\mathbb{Z}\left[T_{1}, \ldots, T_{n}\right]$ and $M_{0}=A_{0}^{n}$ with $f_{0}: M_{0} \rightarrow A_{0}$ given by $a \mapsto \sum a_{i} T_{i}$. Let $I_{0}=f_{0}\left(M_{0}\right)$. We consider the homomorphism $A_{0} \rightarrow A$ defined by $T_{i} \mapsto x_{i}$. Let $B=\mathscr{D}_{A}(I)$ and $B_{0}=\mathscr{D}_{A_{0}}\left(I_{0}\right)$ be the divided power envelopes. Since $f$ and $f_{0}$ are regular, we have $I=I_{0} \otimes_{A} A_{0}$. As in [Berthelot 1974, (3.4.8)] it follows that $B=B_{0} \otimes_{A_{0}} A$. Using Lemma A.2, we get $B=\underline{\lim } M_{r}$ with $M_{r}=M_{0, r} \otimes_{A_{0}} A$. Moreover, since $\operatorname{Tor}_{1}^{A_{0}}\left(A_{0} / I_{0}, A\right)=0$, we obtain exact sequences of $A$-modules

$$
0 \longrightarrow J_{r} \longrightarrow M_{r} \longrightarrow N_{r} \longrightarrow 0
$$

with $J_{r}=J_{0, r} \otimes_{A_{0}} A$ and $N_{r}=N_{0, r} \otimes_{A_{0}} A$, and we obtain filtrations of $N_{r}$ with quotients isomorphic to $A / I$. Similarly there are exact sequences of $A$-modules

$$
0 \longrightarrow J_{r} \longrightarrow A \longrightarrow A / J_{r} \longrightarrow 0
$$

and filtrations of $A / J_{r}$ with quotients isomorphic to $A / I$. Since $A$ and $A / I$ are flat over $R$, it follows that $J_{r}$ and $M_{r}$ and $B$ are flat over $R$. 
We will use the following example of regular immersions.

Lemma A.3. For a ring $R$ and a projective $R$-module $T$ of finite type we consider the complete symmetric algebra $A=R \llbracket T \rrbracket=\prod_{n \geq 0} \operatorname{Sym}_{R}^{n}(T)$ and $M=T \otimes_{R} A$. Then the homomorphism $f: M \rightarrow A$ given by $t \otimes a \mapsto t a$ is regular.

Proof. The complex $K_{*}(M, f)$ is the direct product over $m \geq 0$ of complexes $K_{*}^{(m)}$ with $K_{n}^{(m)}=\Lambda^{n} T \otimes_{R} \operatorname{Sym}^{m-n}(T)$, using the convention $\operatorname{Sym}^{r}(T)=0$ for $r<0$. Since the complexes $K_{*}^{(m)}$ are compatible with base change in $R$, the general case can be reduced to the case where $T$ is free. Then an $R$-basis of $T$ is a regular sequence in $A$, and the assertion follows.

\section{Acknowledgements}

The author thanks Xavier Caruso, Tyler Lawson, and Thomas Zink for interesting and helpful conversations, and the anonymous referee for a very careful reading of the manuscript.

\section{References}

[Berthelot 1974] P. Berthelot, Cohomologie cristalline des schémas de caractéristique $p>0$, Lecture Notes in Mathematics 407, Springer, New York, 1974. MR 52 \#5676 Zbl 0298.14012

[Berthelot et al. 1982] P. Berthelot, L. Breen, and W. Messing, Théorie de Dieudonné cristalline, II, Lecture Notes in Mathematics 930, Springer, Berlin, 1982. MR 85k:14023 Zbl 0516.14015

[Bourbaki 1983] N. Bourbaki, Algèbre commutative: Chapitre 8: Dimension; Chapitre 9: Anneaux locaux noethériens complets, Masson, Paris, 1983. MR 86j:13001 Zbl 0579.13001

[Breuil 1998] C. Breuil, "Schémas en groupes et corps des normes", unpublished manuscript, 1998, Available at http://www.math.u-psud.fr/ breuil/PUBLICATIONS/groupesnormes.pdf.

[Grothendieck 1974] A. Grothendieck, Groupes de Barsotti-Tate et cristaux de Dieudonné, Les Presses de l'Université de Montréal, Montreal, Que., 1974. Séminaire de Mathématiques Supérieures, No. 45 (Été, 1970). MR 54 \#5250 Zbl 0331.14021

[Illusie 1985] L. Illusie, "Déformations de groupes de Barsotti-Tate (d'après A. Grothendieck)", pp. 151-198 in Séminaire sur les pinceaux arithmétiques: la conjecture de Mordell (Paris, 1983/84), edited by L. Szpiro, Astérisque 127, 1985. MR 801922 Zbl 1182.14050

[Katz 1981] N. Katz, "Serre-Tate local moduli”, pp. 138-202 in Surfaces algébriques (Orsay, 197678), edited by J. Giraud et al., Lecture Notes in Math. 868, Springer, Berlin, 1981. MR 83k:14039b Zbl 0477.14007

[Kim 2012] W. Kim, "The classification of p-divisible groups over 2-adic discrete valuation rings", Math. Res. Lett. 19:1 (2012), 121-141. MR 2923180 Zbl 1284.14056

[Kisin 2006] M. Kisin, "Crystalline representations and F-crystals", pp. 459-496 in Algebraic geometry and number theory, edited by V. Ginzburg, Progr. Math. 253, Birkhäuser, Boston, 2006. MR 2007j:11163 Zbl 1184.11052

[Kisin 2009] M. Kisin, "Modularity of 2-adic Barsotti-Tate representations", Invent. Math. 178:3 (2009), 587-634. MR 2010k:11089

[Lau 2008] E. Lau, "Displays and formal p-divisible groups", Invent. Math. 171:3 (2008), 617-628. MR 2009j:14058 Zbl 1186.14048 
[Lau 2009] E. Lau, "A duality theorem for Dieudonné displays", Ann. Sci. Éc. Norm. Supér. (4) 42:2 (2009), 241-259. MR 2010d:14065 Zbl 1182.14051

[Lau 2010] E. Lau, "Frames and finite group schemes over complete regular local rings", Doc. Math. 15 (2010), 545-569. MR 2011g:14107 Zbl 1237.14053

[Lau 2012] E. Lau, "Displayed equations for Galois representations", preprint, 2012. arXiv 1012.4436

[Lau 2013] E. Lau, "Smoothness of the truncated display functor", J. Amer. Math. Soc. 26:1 (2013), 129-165. MR 2983008 Zbl 1273.14040

[Liu 2013] T. Liu, "The correspondence between Barsotti-Tate groups and Kisin modules when $p=2$, J. Théor. Nombres Bordeaux 25:3 (2013), 661-676. MR 3179680 Zbl 06291371

[Mazur and Messing 1974] B. Mazur and W. Messing, Universal extensions and one dimensional crystalline cohomology, Lecture Notes in Mathematics 370, Springer, Berlin, 1974. MR 51 \#10350 Zbl 0301.14016

[Messing 1972] W. Messing, The crystals associated to Barsotti-Tate groups: with applications to abelian schemes, Lecture Notes in Mathematics 264, Springer, Berlin, 1972. MR 50 \#337 Zbl 0243.14013

[Schlessinger 1968] M. Schlessinger, "Functors of Artin rings", Trans. Amer. Math. Soc. 130 (1968), 208-222. MR 36 \#184 Zbl 0167.49503

[SGA 1970] M. Demazure and A. Grothendieck (editors), Schémas en groupes, I: Propriétés générales des schémas en groupes (Séminaire de Géométrie Algébrique du Bois Marie 1962/64 = SGA 3 I), Lecture Notes in Math. 151, Springer, Berlin, 1970. MR 43 \#223a Zbl 0207.51401

[SGA 1971] P. Berthelot, A. Grothendieck, and L. Illusie (editors), Théorie des intersections et théorème de Riemann-Roch (Séminaire de Géométrie Algébrique du Bois Marie 1966/67 = SGA 6), Lecture Notes in Math. 225, Springer, Berlin, 1971. MR 50 \#7133 Zbl 0218.14001

[Vasiu and Zink 2010] A. Vasiu and T. Zink, "Breuil's classification of $p$-divisible groups over regular local rings of arbitrary dimension", pp. 461-479 in Algebraic and arithmetic structures of moduli spaces (Sapporo, Japan, 2007), edited by I. Nakamura and L. Weng, Adv. Stud. Pure Math. 58, Math. Soc. Japan, Tokyo, 2010. MR 2012a:14101 Zbl 1210.14049

[Viehmann and Wedhorn 2013] E. Viehmann and T. Wedhorn, "Ekedahl-Oort and Newton strata for Shimura varieties of PEL type", Math. Ann. 356:4 (2013), 1493-1550. MR 3072810 Zbl 06194422

[Zink 2001a] T. Zink, “A Dieudonné theory for p-divisible groups”, pp. 139-160 in Class field theory - its centenary and prospect (Tokyo, 1998), edited by K. Miyake, Adv. Stud. Pure Math. 30, Math. Soc. Japan, Tokyo, 2001. MR 2002h:14075 Zbl 1052.14048

[Zink 2001b] T. Zink, "Windows for displays of p-divisible groups", pp. 491-518 in Moduli of abelian varieties (Texel Island, 1999), edited by C. Faber et al., Progr. Math. 195, Birkhäuser, Basel, 2001. MR 2002c:14073 Zbl 1099.14036

[Zink 2002] T. Zink, "The display of a formal p-divisible group", pp. 127-248 in Cohomologies p-adiques et applications arithmétiques, I, edited by P. Berthelot et al., Astérisque 278, Société Mathématique de France, 2002. MR 2004b:14083 Zbl 1008.14008

Communicated by Mark Kisin

Received 2014-03-07 Revised 2014-09-22 Accepted 2014-10-28

elau@math.upb.de

Institut für Mathematik, Universität Paderborn,

D-33098 Paderborn, Germany 


\section{Algebra \& Number Theory}

msp.org/ant

\section{EDITORS}

MANAGING EDITOR

Bjorn Poonen

Massachusetts Institute of Technology

Cambridge, USA

\author{
EDITORIAL BOARD CHAIR \\ David Eisenbud \\ University of California \\ Berkeley, USA
}

BOARD OF EDITORS

Georgia Benkart

Dave Benson

Richard E. Borcherds

John H. Coates

J-L. Colliot-Thélène

Brian D. Conrad

Hélène Esnault

Hubert Flenner

Edward Frenkel

Andrew Granville

Joseph Gubeladze

Roger Heath-Brown

Craig Huneke

János Kollár

Yuri Manin

Barry Mazur

Philippe Michel

Susan Montgomery
University of Wisconsin, Madison, USA

University of Aberdeen, Scotland

University of California, Berkeley, USA

University of Cambridge, UK

CNRS, Université Paris-Sud, France

University of Michigan, USA

Freie Universität Berlin, Germany

Ruhr-Universität, Germany

University of California, Berkeley, USA

Université de Montréal, Canada

San Francisco State University, USA

Oxford University, UK

University of Virginia, USA

Princeton University, USA

Northwestern University, USA

Harvard University, USA

École Polytechnique Fédérale de Lausanne

University of Southern California, USA
Shigefumi Mori

Raman Parimala

Jonathan Pila

Anand Pillay

Victor Reiner

Peter Sarnak

Joseph H. Silverman

Michael Singer

Vasudevan Srinivas

J. Toby Stafford

Bernd Sturmfels

Richard Taylor

Ravi Vakil

Michel van den Bergh

Marie-France Vignéras

Kei-Ichi Watanabe

Efim Zelmanov

Shou-Wu Zhang
RIMS, Kyoto University, Japan

Emory University, USA

University of Oxford, UK

University of Notre Dame, USA

University of Minnesota, USA

Princeton University, USA

Brown University, USA

North Carolina State University, USA

Tata Inst. of Fund. Research, India

University of Michigan, USA

University of California, Berkeley, USA

Harvard University, USA

Stanford University, USA

Hasselt University, Belgium

Université Paris VII, France

Nihon University, Japan

University of California, San Diego, USA

Princeton University, USA

\section{PRODUCTION}

production@msp.org

Silvio Levy, Scientific Editor

See inside back cover or msp.org/ant for submission instructions.

The subscription price for 2014 is US \$225/year for the electronic version, and $\$ 400 /$ year ( $\$ 55$, if shipping outside the US) for print and electronic. Subscriptions, requests for back issues and changes of subscribers address should be sent to MSP.

Algebra \& Number Theory (ISSN 1944-7833 electronic, 1937-0652 printed) at Mathematical Sciences Publishers, 798 Evans Hall \#3840, c/o University of California, Berkeley, CA 94720-3840 is published continuously online. Periodical rate postage paid at Berkeley, CA 94704, and additional mailing offices.

ANT peer review and production are managed by EditFLOW ${ }^{\circledR}$ from MSP.

\section{PUBLISHED BY}

mathematical sciences publishers

nonprofit scientific publishing

http://msp.org/

(C) 2014 Mathematical Sciences Publishers 


\section{Algebra \& Number Theory}

Volume $8 \quad$ No. $9 \quad 2014$

Zeros of $L$-functions outside the critical strip

ANDREW R. BOOKER and FRANK THORNE

Tropical independence I: Shapes of divisors and a proof of the Gieseker-Petri theorem DAVID JeNSEN and SAM PAYNE

New equidistribution estimates of Zhang type

D. H. J. POLYMATH

2043

2067

Relations between Dieudonné displays and crystalline Dieudonné theory

2201

EIKE LAU

Finiteness of unramified deformation rings

2263

Patrick B. Allen and Frank Calegari

2273

On direct images of pluricanonical bundles

Minnea Popa and Christian SchNell 